\title{
STEPS TOWARD DETERMINATION OF THE SIZE AND STRUCTURE OF THE BROAD-LINE REGION IN ACTIVE GALACTIC NUCLEI. VIII. AN INTENSIVE HST, IUE, AND GROUND-BASED STUDY OF NGC 5548
}

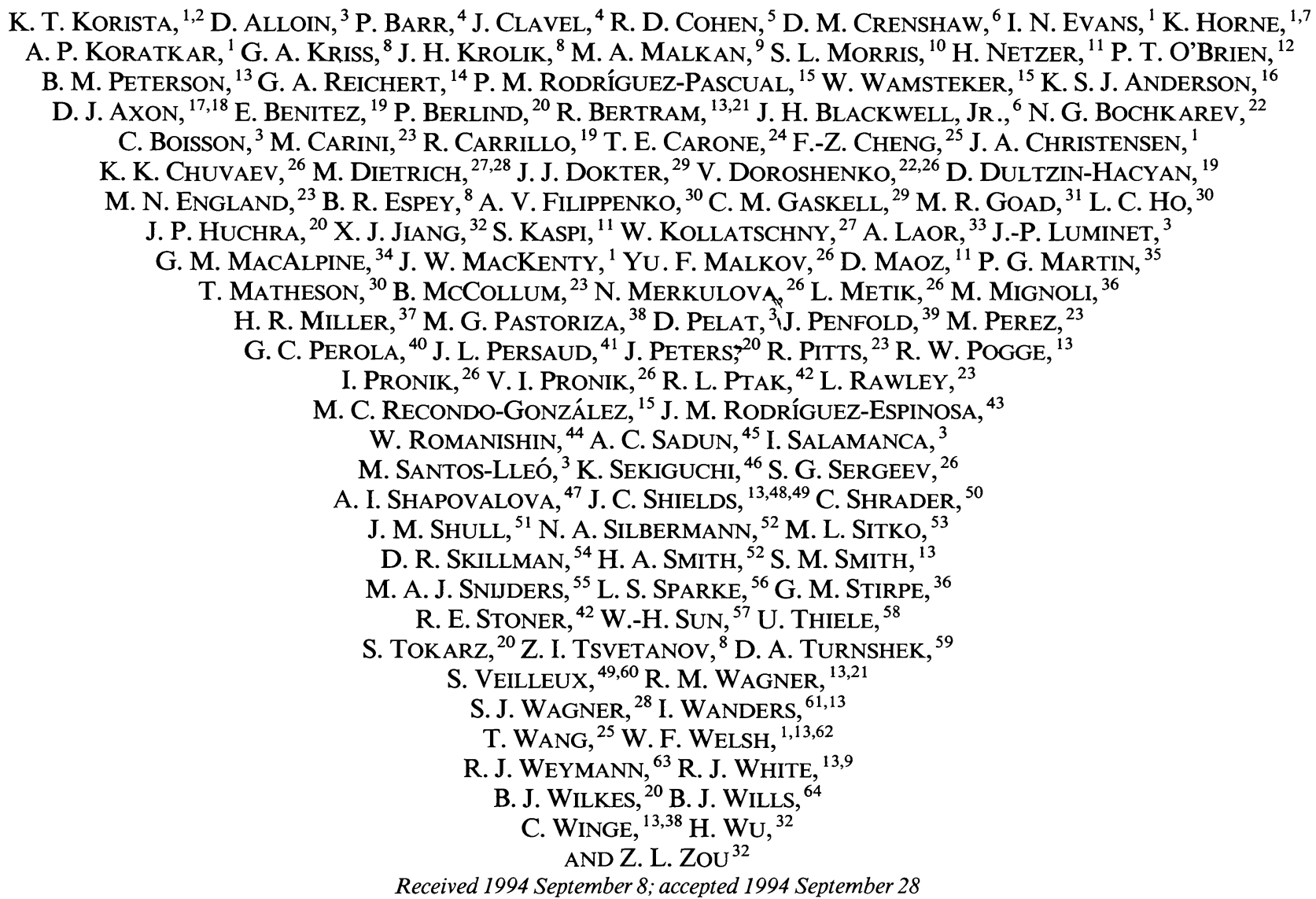

\footnotetext{
${ }^{1}$ Space Telescope Science Institute, 3700 San Martin Drive, Baltimore, MD 21218.

${ }^{2}$ Present address: Department of Physics and Astronomy, University of Kentucky, Lexington, KY 40506; korista@pa.uky.edu.

${ }^{3}$ Observatoire de Paris, URA 173 CNRS, 92195 Meudon, France.

${ }^{4}$ ISO Observatory, Astrophysics Division of ESA, ESTEC, Postbus 299, 2200-AG, The Netherlands.

${ }^{5}$ Center for Astrophysics and Space Science, C-011, University of California at San Diego, 9500 Gillman Drive, La Jolla, CA 92093-0111.

${ }^{6}$ Astronomy Program, Computer Sciences Corporation, NASA Goddard Space Flight Center, Code 681, Greenbelt, MD 20771.

${ }^{7}$ Sterrekundig Instituut, University of Utrecht, P.O. Box 80000, NL-3508 TA, Utrecht, The Netherlands.

${ }^{8}$ Department of Physics and Astronomy, Johns Hopkins University, Baltimore, MD 21218.

${ }^{9}$ Department of Astronomy, University of California, Math-Science Building, Los Angeles, CA 90024.

${ }^{10}$ Dominion Astrophysical Observatory, 5071 West Saanich Road, Victoria, BC, Canada V8X 4M6.

${ }^{11}$ School of Physics and Astronomy and Wise Observatory, Tel-Aviv University, Tel Aviv 69978, Israel.

${ }^{12}$ Department of Astrophysics, Oxford University, Keble Road, Oxford OX1 3RH, England, UK

${ }^{13}$ Department of Astronomy, Ohio State University, 174 West 18 th Avenue, Columbus, OH 43210.

${ }^{14}$ Universities Space Research Association, NASA Goddard Space Flight Center, Code 668, Greenbelt, MD 20771.

${ }^{15}$ ESA IUE Observatory, P.O. Box 50727, 28080 Madrid, Spain.

${ }^{16}$ Department of Astronomy, New Mexico State University, Box 30001, Department 4500, Las Cruces, NM 88003.

${ }^{17}$ Nuffield Radio Astronomy Laboratories, University of Manchester, Macclesfield, Cheshire, SK 11 9DL, England, UK.

${ }^{18}$ European Space Agency, Space Telescope Science Institute, 3700 San Martin Drive, Baltimore, MD 21218.

${ }^{19}$ Universidad Nacional Autónoma de México, Instituto de Astronomia, Apartado Postal 70-264, 04510 México D.F., Mexico.

${ }^{20}$ Harvard-Smithsonian Center for Astrophysics, 60 Garden Street, Cambridge, MA 02138.

${ }^{21}$ Postal address: Lowell Observatory, Mars Hill Road, 1400 West, Flagstaff, AZ 86001.

${ }^{22}$ Sternberg Astronomical Institute, University of Moscow, Universitetskij prosp. 13, Moscow V-234, Russia.

${ }^{23}$ Computer Sciences Corporation, NASA Goddard Space Flight Center, Code 684.9, Greenbelt, MD 20771.

${ }^{24}$ Space Sciences Laboratory, University of California at Berkeley, Berkeley, CA 94720.
} 


\section{ABSTRACT}

We present the data and initial results from a combined HST/IUE/ground-based spectroscopic monitoring campaign on the Seyfert 1 galaxy NGC 5548 that was undertaken in order to address questions that require both higher temporal resolution and higher signal-to-noise ratios than were obtained in our previous multiwavelength monitoring of this galaxy in 1988-1989. IUE spectra were obtained once every 2 days for a period of 74 days beginning on 1993 March 14. During the last 39 days of this campaign, spectroscopic observations were also made with the HST Faint Object Spectrograph (FOS) on a daily basis. Ground-based observations, consisting of 165 optical spectra and 77 photometric observations (both CCD imaging and aperture photometry), are reported for the period 1992 October-1993 September, although many of the data are concentrated around the time of the satellite-based program. These data constitute a fifth year of intensive optical monitoring of this galaxy. In this contribution we describe the acquisition and reduction of all of the satellite and ground-based data obtained in this program. We describe in detail various photometric problems with the FOS and explain how we identified and corrected for various anomalies.

During the HST portion of the monitoring campaign, the $1350 \AA$ continuum flux is found to have varied by nearly a factor of 2 . In other wave bands, the continuum shows nearly identical behavior, except that the amplitude of variability is larger at shorter wavelengths, and the continuum light curves appear to show more shorttimescale variability at shorter wavelengths. The broad emission lines also vary in flux, with amplitudes that are slightly smaller than the UV continuum variations and with a small time delay relative to the UV continuum. On the basis of simple time-series analysis of the UV and optical continuum and emission-line light curves, we find (1) that the ultraviolet and optical continuum variations are virtually simultaneous, with any lag between the $1350 \AA$ continuum and the $5100 \AA$ continuum amounting to less than about 1 day; (2) that the variations in the highest ionization lines observed, He II $\lambda 1640$ and $N$ v $\lambda 1240$, lag behind the continuum variations by somewhat

${ }^{25}$ Center for Astrophysics, University of Science and Technology, Hefei, Anhui, People's Republic of China.

${ }^{26}$ Crimean Astrophysical Observatory, P/O Nauchny, 334413 Crimea, Ukraine.

${ }^{27}$ Universitäts-Sternwarte Göttingen, Geismarlandstrasse 11, D-37083 Göttingen, Germany.

${ }^{28}$ Landessternwarte, Königstuhl, D-69117 Heidelberg, Germany.

${ }^{29}$ Department of Physics and Astronomy, University of Nebraska, Lincoln, NE 68588.

${ }^{30}$ Department of Astronomy, University of California at Berkeley, Berkeley, CA 94720.

${ }^{31}$ Department of Physics and Astronomy, University College London, Gower Street, London WC1E 6BT, England, UK.

${ }^{32}$ Beijing Astronomical Observatory, Chinese Academy of Sciences, Zhongguancun, Beijing, People's Republic of China.

${ }^{33}$ Department of Astronomy, Caltech 130-33, Pasadena, CA 91125.

${ }^{34}$ Department of Astronomy, University of Michigan, Dennison Building, Ann Arbor, MI 48109.

${ }^{35}$ Canadian Institute for Theoretical Astrophysics, University of Toronto, Toronto, ON, Canada M5S 1A1.

${ }^{36}$ Osservatorio Astronomico di Bologna, via Zamboni 33, I-40126, Bologna, Italy.

${ }^{37}$ Department of Physics and Astronomy, Georgia State University, Atlanta, GA 30303.

${ }^{38}$ Departamento de Astronomia, Instituto de Física, Universidade Federal do Rio Grande do Sul, Avenida Bento Gonçalves, 9500, CP15051, CEP 91500 , Porto Alegre, RS, Brazil.

${ }^{39}$ Department of Physics and Astronomy, University of Calgary, 2500 University Drive NW, Calgary, Canada AB T2N 1N4; and Department of Mathematics, Physics, and Engineering, Mount Royal College, Calgary, Canada T3E 6K6.

${ }^{40}$ Istituto Astronomico dell'Università, via Lancisi 29, I-00161 Rome, Italy.

${ }^{41}$ Institute of Astronomy, Madingley Road, Cambridge CB3 OHA, England, UK.

${ }^{42}$ Department of Physics and Astronomy, Bowling Green State University, Bowling Green, OH 43403.

${ }^{43}$ Instituto de Astrofísica de Canarias, E-38200 La Laguna, Tenerife, Spain.

${ }^{44}$ Department of Physics and Astronomy, University of Oklahoma, Norman, OK 73019.

${ }^{45}$ Department of Physics and Astronomy and Bradley Observatory, Agnes Scott College, Decatur, GA 30030.

${ }^{46}$ South African Astronomical Observatory, P.O. Box 9, Observatory 7935, South Africa.

${ }^{47}$ Special Astrophysical Observatory, Russian Academy of Science, Nizhny Arkhyz, Stavropolsky Kraj, 357140, Russia.

${ }^{48}$ Steward Observatory, University of Arizona, Tucson, AZ 85721.

${ }^{49}$ Hubble Fellow.

${ }^{50}$ Computer Sciences Corporation, GRO Science Support Center, NASA Goddard Space Flight Center, Code 668.1, Greenbelt, MD 20771.

51 Joint Institute for Laboratory Astrophysics and National Institute of Standards and Technology, University of Colorado, Campus Box 440, Boulder, CO 80309.

${ }_{52}^{5}$ Department of Physics and Astronomy, Michigan State University, East Lansing, MI 48824.

${ }^{53}$ Department of Physics, M.L. 11, University of Cincinnati, 400 Geology/Physics Building, Cincinnati, OH 45221.

${ }^{54}$ Center for Basement Astrophysics, 9517 Washington Avenue, Laurel, MD 20723.

${ }^{55}$ IRAM, 300 Rue de la Piscine, 38046 Saint Martin d'Heres, France.

${ }^{56}$ Department of Astronomy, University of Wisconsin, 475 North Charter Street, Madison, WI 53706.

${ }^{57}$ Institute of Astronomy, National Central University, Chung-Li, Taiwan 32054, Republic of China.

${ }^{58}$ Max-Planck-Institut für Astronomie, Königstuhl, D-69117 Heidelberg, Germany.

${ }^{59}$ Department of Physics and Astronomy, University of Pittsburgh, Pittsburgh, PA 15260.

${ }^{60} \mathrm{Kitt}$ Peak National Observatory, National Optical Astronomy Observatories, P.O. Box 26732, Tucson, AZ 85726

${ }^{61}$ Astronomiska Observatoriet, Box 515, S-751 20 Uppsala, Sweden.

62 Department of Physics, Keele University, Keele ST5 5BG, Staffordshire, England, UK.

${ }^{63}$ Observatories of the Carnegie Institution of Washington, 813 Santa Barbara Street, Pasadena, CA 91101.

${ }^{64}$ McDonald Observatory and Department of Astronomy, University of Texas, RLM 15.308, Austin, TX 78712. 
less than 2 days; and ( 3 ) that the velocity field of the C IV-emitting region is not dominated by radial motion. The results on the $\mathrm{C}$ IV velocity field are preliminary and quite uncertain, but there are some weak indications that the emission-line wings $\left(|\Delta v| \geq 3000 \mathrm{~km} \mathrm{~s}^{-1}\right)$ respond to continuum variations slightly more rapidly than does the core. The optical observations show that the variations in the broad $\mathrm{H} \beta$ line flux follow the continuum variations with a time lag of around 2 weeks, about twice the lag for $\mathrm{Ly} \alpha$ and $\mathrm{C}$ IV, as in our previous monitoring campaign on this same galaxy. However, the lags measured for $\operatorname{Ly} \alpha, \mathrm{C}$ IV, and $\mathrm{H} \beta$ are each slightly smaller than previous determinations. We confirm two trends reported earlier, namely, (1) that the UV/optical continuum becomes "harder" as it gets brighter and (2) that the highest ionization emission lines have the shortest lags, thus indicating radial ionization stratification of a broad-line region that spans over an order of magnitude range in radius.

Subject headings: galaxies: active — galaxies: individual (NGC 5548) — galaxies: nuclei — galaxies: photometry — galaxies: Seyfert — ultraviolet: galaxies

\section{INTRODUCTION}

High temporal frequency monitoring of Seyfert galaxies over extended periods has proved to be a powerful way to unravel the structure and physical conditions of the broad-line region (BLR) in active galactic nuclei (AGNs). The photoionized BLR gas responds to the variations in the energy input rate from the continuum source with a delay determined by the light-travel time across the BLR and the geometry of the region. Thus, determination of the line response provides a direct means of mapping the BLR emissivity distribution, effectively providing spatial resolution on microarcsecond scales. By measuring the response for lines of different ionization stages and comparing the results from model photoionization calculations, one is able to infer the run of physical conditions as a function of radius. Such measurements are of fundamental importance, as they are the only way to determine the photon number density incident on the line-emitting gas.

At any position within the BLR, the emission-line flux at some time $t$ is the response to the ionizing continuum flux generated at some previous time $t-\tau$, where $\tau$ is set by the lighttravel time from the continuum source to that position. Under the usual assumptions (see Peterson 1993 for a recent discussion), the emission-line light curve $L(t)$ over the entire BLR is the convolution of the observed continuum light curve $C(t)$ with a transfer function $\Psi(\tau)$, i.e.,

$$
L(t)=\int_{-\infty}^{\infty} \Psi(\tau) C(t-\tau) d \tau
$$

where $\Psi(\tau)$ is determined by the responsivity-weighted geometrical distribution of the line-emitting gas (Blandford \& McKee 1982). A spectroscopic data set of sufficient signal-tonoise ratio, temporal resolution, and duration to sample wide variations in the continuum variability is required to invert the integral and solve for $\Psi(\tau)$. The transfer function can also be solved for using light curves at different velocities $v$ across the line profile, yielding a two-dimensional map $\Psi(v, \tau)$ of the BLR. This extra dimension provides the information necessary to break the near-degeneracy in some one-dimensional transfer function solutions, allowing for a less ambiguous determination of the responsivity-weighted distribution of the BLR gas in phase space.

The bright Seyfert 1 galaxy NGC 5548 has received considerable attention in these efforts. Beginning in 1988 December, the International Cltraviolet Explorer (IUE) observed this AGN once every 4 days for an 8 month period (Clavel et al. 1991, hereafter Paper I). The optical spectral variability was monitored with a concurrent ground-based campaign (Peterson et al. 1991, 1992 and Dietrich et al. 1993, hereafter Papers II-IV, respectively). Krolik et al. (1991), Horne, Welsh, \& Peterson (1991), and Ferland et al. (1992) derived and investigated the one-dimensional transfer functions of the strongest UV lines and $\mathrm{H} \beta$. By combining the UV and optical results, Maoz et al. (1993) analyzed the variability of the "small blue bump," a blend of ultraviolet Fe II and Balmer continuum emission. More recently, the southern hemisphere Seyfert 1 galaxy NGC 3783 was similarly monitored (Reichert et al. 1994 and Stirpe et al. 1994, hereafter Papers V and VI, respectively). Peterson et al. (1994, hereafter Paper VII) analyzed the optical continuum and $\mathrm{H} \beta$ emission-line variability of NGC 5548 over the 4 year period from 1988 December through 1992 October.

Peterson (1993) reviewed in detail the results of the past monitoring campaigns of NGC 5548. As in many successful experiments, important new questions arose whose answers required additional data; in this particular case not only better temporal sampling than was achieved in the original campaign (4 days) but higher signal-to-noise ratios as well. An intensive ground-based and ultraviolet IUE and Hubble Space Telescope ( $H S T)$ monitoring campaign of NGC 5548 was recently undertaken to address three key unanswered questions from the original IUE and ground-based campaigns of 1988-1989. These are the following:

1. Is there a phase difference between the UV and optical continuum variations? The establishment of limits on whether the variations in the different wave bands are truly simultaneous can provide a fundamental constraint for models of the continuum emission.

2. What is the response time of the most rapidly varying high-ionization lines? The rapid variability of the highest ionization lines indicates that there is a $\mathrm{He}^{+}-\mathrm{He}^{++}$ionization front within a few light days of the continuum source.

3. What is the velocity field of the BLR? Determination of the velocity field provides some of the strongest possible constraints on the origin of the BLR and physical conditions within a few light days of the central source, and indeed might also lead to a direct determination of the mass of the central object. The results from the original campaign are ambiguous, with Clavel (1991) arguing for random cloud motions with higher velocities close to the central source and Crenshaw \& Blackwell (1990) arguing for gravitational infall.

In $\S 2$ we outline the $H S T$ observations, in $\S 3$ the IUE observations, and in $\S 4$ the fifth year of the ground-based cam- 
paign, during which the $H S T$ and IUE observations were carried out. We describe the intercalibration of the various data sets and their measurements in $\S 5$. The variability is characterized and the cross-correlation functions are calculated in $\S 6$. We summarize our results in $\S 7$.

\section{THE $H S T /$ FOS SPECTRA}

\subsection{The FOS Observations}

Using the blue-side detector of the HST Faint Object Spectrograph (FOS), NGC 5548 was observed every day from 1993 April 19 through 1993 May 27, a total of 39 separate visits each separated by approximately $24 \mathrm{hr}$. The nucleus was centered in the 4"3 square aperture, using a three-stage "peak-up" routine. The mode of the target-centering error distribution is about 0 ".175 (but note that the error distribution is highly nonGaussian, falling off steeply at larger miscenterings). As the Digicon diodes project to 1 ".4 perpendicular to the dispersion, the effective aperture for these data is 4 ."3 3 1".4. Such a large aperture admits the broad wings of the point spread function (PSF) due to spherical aberration in the primary mirror. The effects of the PSF wings on the spectrophotometry due to miscenterings of the object on the diode array will be discussed briefly in later sections. All observations used two guide stars, one in each of the two functioning fine guidance sensors. Because of a malfunction of the solar arrays just prior to this campaign, the position angle (P.A.) of the telescope was allowed to roll, whereas the original proposal called for a constant P.A. to minimize photometric uncertainties due to any possible nonnuclear sources of light. On 26 visits, the $\mathrm{G} 190 \mathrm{H}$ grating exposure of $1295 \mathrm{~s}$ occurred in the same orbit (second) as the Iast stage in the peak-up; in the other 13 visits, the three-stage peak-up was completed entirely in the first orbit with the G190H exposure occurring after a reacquisition in the second. The G130H grating exposure of $1750 \mathrm{~s}$ followed after a reacquisition (re-centering upon guide stars) in the third orbit.

The FOS detector is a Digicon, with a linear 512 diode array. The dispersed photons strike a photocathode which produces electrons, which are then accelerated onto the diode array to be read out. The electron trajectories are magnetically focused to map the photocathode surface onto the Digicon array. The G190H observations consisted of five separate readouts, each of 259 s duration, while the $\mathrm{G} 130 \mathrm{H}$ observations consisted of seven separate $250 \mathrm{~s}$ readouts. Each readout was summed with the previous one after correcting for the effects of the geomagnetically induced image-motion problem (GIMP) (Junkkarinen et al. 1991; Fitch et al. 1993). GIMP compensation was performed in real time on board the spacecraft. The on-board GIMP correction was done in directions both along and perpendicular to the diode array. In order to oversample the response of each diode and to minimize the effects of dead diodes, the standard exposure technique of quarter-stepping with an overscan of five diodes was used. This produces an array of 2064 pixels, each pixel having an effective exposure time of $323.75 \mathrm{~s}$ for the $\mathrm{G} 190 \mathrm{H}$ and $437.5 \mathrm{~s}$ for the $\mathrm{G} 130 \mathrm{H}$, or one-quarter of the total integration time in each case. The data in the first and last 20 pixels have diminishing signal-to-noise ratios as a result of the overscanning technique; grating efficiencies drop near the ends of spectra as well. Figure 1 shows the signal-to-noise ratio per pixel for the full integration on the object during the lowest observed overall flux level of the campaign for both gratings. The square-bottomed "absorption" features are the result of the bad diodes in the array. Table 1 gives a $\log$ of the FOS observations. Columns (1)-(8) are the

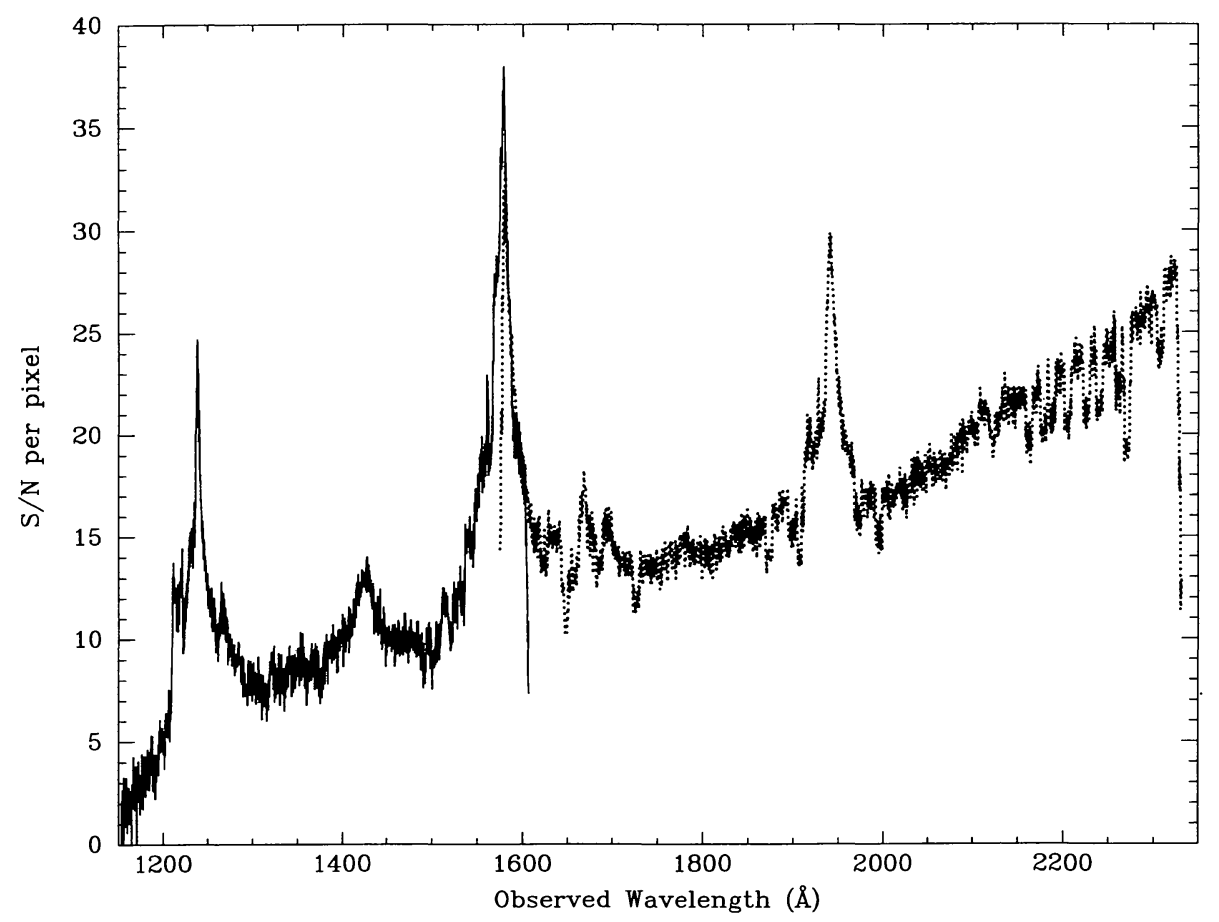

FIG. 1.-G130H (solid line) and G190H (dotted line) signal-to-noise ratio per pixel as a function of wavelength for the first day in the FOS campaign, JD 2,449,097. 
TABLE 1

LOG OF FOS SPECTROSCOPIC OBSERVATIONS

\begin{tabular}{|c|c|c|c|c|c|c|c|}
\hline \multirow{2}{*}{$\begin{array}{l}\text { FOS Campaign } \\
\text { Day Number } \\
\text { (1) }\end{array}$} & \multicolumn{2}{|l|}{ UT } & \multirow{2}{*}{$\begin{array}{c}\text { Julian Date } \\
(2440000+) \\
(4)\end{array}$} & \multirow[b]{2}{*}{$\begin{array}{c}\text { Grating } \\
\text { (5) }\end{array}$} & \multirow[b]{2}{*}{$\begin{array}{l}\text { P.A. } \\
(6)\end{array}$} & \multirow[b]{2}{*}{$\begin{array}{l}\text { Comments } \\
\text { (7) }\end{array}$} & IRAF \\
\hline & $\begin{array}{l}\text { Date } \\
(2)\end{array}$ & $\begin{array}{l}\text { Time } \\
(3)\end{array}$ & & & & & $\begin{array}{l}\text { file name } \\
\text { (8) }\end{array}$ \\
\hline 01 & 1993 Apr 19 & $11: 23: 36$ & 9096.97 & $\mathrm{G} 190 \mathrm{H}$ & 4 & & n59097 \\
\hline 01 & 1993 Apr 19 & $12: 44: 28$ & 9097.03 & G130H & 4 & & n59097 \\
\hline 02 & 1993 Apr 20 & $12: 52: 34$ & 9098.04 & $\mathrm{G} 190 \mathrm{H}$ & 2 & & n59098 \\
\hline 02 & 1993 Арr 20 & $14: 26: 42$ & 9098.10 & $\mathrm{G} 130 \mathrm{H}$ & 2 & & n59098 \\
\hline 03 & 1993 Apr 21 & $11: 35: 14$ & 9098.98 & G190H & 0 & & n59099 \\
\hline 03 & 1993 Apr 21 & $12: 56: 06$ & 9099.04 & $\mathrm{G} 130 \mathrm{H}$ & 0 & D & n59099 \\
\hline 04 & 1993 Apr 22 & $11: 40: 59$ & 9099.98 & G190H & 359 & & n59100 \\
\hline 04 & 1993 Apr 22 & $13: 01: 50$ & 9100.04 & $\mathrm{G} 130 \mathrm{H}$ & 359 & & n59100 \\
\hline 05 & 1993 Apr 23 & $11: 46: 45$ & 9100.99 & $\mathrm{G} 190 \mathrm{H}$ & 357 & & n59101 \\
\hline 05 & 1993 Apr 23 & $13: 07: 35$ & 9101.04 & $\mathrm{G} 130 \mathrm{H}$ & 357 & & n59101 \\
\hline 06 & 1993 Apr 24 & $11: 52: 27$ & 9101.99 & $\mathrm{G} 190 \mathrm{H}$ & 356 & & n59102 \\
\hline 06 & 1993 Apr 24 & $13: 13: 18$ & 9102.05 & $\mathrm{G} 130 \mathrm{H}$ & 356 & & n59102 \\
\hline 07 & 1993 Apr 25 & $13: 21: 17$ & 9103.05 & G190H & 354 & & n59103 \\
\hline 07 & 1993 Apr 25 & $14: 55: 25$ & 9103.12 & $\mathrm{G} 130 \mathrm{H}$ & 354 & $\mathrm{D}$ & n59103 \\
\hline 08 & 1993 Apr 26 & $12: 03: 55$ & 9104.00 & G190H & 353 & & n59104 \\
\hline 08 & 1993 Apr 26 & $13: 24: 47$ & 9104.05 & $\mathrm{G} 130 \mathrm{H}$ & 353 & & n59104 \\
\hline 09 & 1993 Apr 27 & $11: 56: 23$ & 9105.00 & $\mathrm{G} 190 \mathrm{H}$ & 351 & & n59105 \\
\hline 09 & 1993 Apr 27 & $13: 30: 32$ & 9105.06 & $\mathrm{G} 130 \mathrm{H}$ & 351 & & n59105 \\
\hline 10 & 1993 Apr 28 & $12: 02: 08$ & 9106.00 & G190H & 350 & & n59106 \\
\hline 10 & 1993 Apr 28 & $13: 36: 16$ & 9106.06 & $\mathrm{G} 130 \mathrm{H}$ & 350 & & n59106 \\
\hline 11 & 1993 Apr 29 & $10: 44: 48$ & 9106.95 & $\mathrm{G} 190 \mathrm{H}$ & 348 & & $\mathrm{n} 59107$ \\
\hline 11 & 1993 Apr 29 & $12: 05: 40$ & 9107.00 & $\mathrm{G} 130 \mathrm{H}$ & 348 & & n59107 \\
\hline 12 & 1993 Арг 30 & $10: 50: 37$ & 9107.95 & G190H & 347 & & n59108 \\
\hline 12 & 1993 Apr 30 & $12: 20: 21$ & 9108.01 & $\mathrm{G} 130 \mathrm{H}$ & 347 & & n59108 \\
\hline 13 & 1993 May 01 & $10: 56: 30$ & 9108.95 & $\mathrm{G} 190 \mathrm{H}$ & 346 & & n59109 \\
\hline 13 & 1993 May 01 & $12: 17: 22$ & 9109.01 & $\mathrm{G} 130 \mathrm{H}$ & 346 & & n59109 \\
\hline 14 & 1993 May 02 & $12: 25: 36$ & 9110.02 & $\mathrm{G} 190 \mathrm{H}$ & 344 & & n59110 \\
\hline 14 & 1993 May 02 & $13: 59: 44$ & 9110.08 & $\mathrm{G} 130 \mathrm{H}$ & 344 & & $\mathrm{n} 59110$ \\
\hline 15 & 1993 May 03 & $11: 08: 27$ & 9110.96 & $\mathrm{G} 190 \mathrm{H}$ & 343 & & n59111 \\
\hline 15 & 1993 May 03 & $12: 29: 19$ & 9111.02 & $\mathrm{G} 130 \mathrm{H}$ & 343 & & n59111 \\
\hline 16 & 1993 May 04 & $11: 14: 34$ & 9111.96 & $\mathrm{G} 190 \mathrm{H}$ & 342 & & n59112 \\
\hline 16 & 1993 May 04 & $12: 35: 27$ & 9112.02 & $\mathrm{G} 130 \mathrm{H}$ & 342 & & n59112 \\
\hline 17 & 1993 May 05 & $11: 20: 49$ & 9112.97 & $\mathrm{G} 190 \mathrm{H}$ & 340 & & n59113 \\
\hline 17 & 1993 May 05 & $12: 41: 42$ & 9113.03 & $\mathrm{G} 130 \mathrm{H}$ & 340 & & n59113 \\
\hline 18 & 1993 May 06 & $11: 27: 11$ & 9113.97 & $\mathrm{G} 190 \mathrm{H}$ & 339 & $U$ & n59114 \\
\hline 18 & 1993 May 06 & $12: 48: 06$ & 9114.03 & $\mathrm{G} 130 \mathrm{H}$ & 339 & $\mathrm{U}$ & n59114 \\
\hline 19 & 1993 May 07 & $11: 20: 26$ & 9114.97 & $\mathrm{G} 190 \mathrm{H}$ & 338 & & n59115 \\
\hline 19 & 1993 May 07 & $12: 54: 38$ & 9115.04 & $\mathrm{G} 130 \mathrm{H}$ & 338 & & n59115 \\
\hline 20 & 1993 May 08 & $11: 27: 06$ & 9115.97 & $\mathrm{G} 190 \mathrm{H}$ & 337 & $\mathrm{U}$ & n59116 \\
\hline 20 & 1993 May 08 & $13: 01: 17$ & 9116.04 & $\mathrm{G} 130 \mathrm{H}$ & 337 & $\mathrm{U}$ & n59116 \\
\hline 21 & 1993 May 09 & $10: 10: 42$ & 9116.92 & $\mathrm{G} 190 \mathrm{H}$ & 335 & $\mathrm{U}$ & n59117 \\
\hline 21 & 1993 May 09 & $11: 31: 38$ & 9116.98 & $\mathrm{G} 130 \mathrm{H}$ & 335 & $\mathrm{U}$ & n59117 \\
\hline 22 & 1993 May 10 & $08: 27: 49$ & 9117.85 & $\mathrm{G} 190 \mathrm{H}$ & 334 & & n59118 \\
\hline 22 & 1993 May 10 & $10: 02: 01$ & 9117.91 & $\mathrm{G} 130 \mathrm{H}$ & 334 & & n59118 \\
\hline 23 & 1993 May 11 & $10: 11: 11$ & 9118.92 & $\mathrm{G} 190 \mathrm{H}$ & 334 & & n59119 \\
\hline 23 & 1993 May 11 & $11: 45: 24$ & 9118.99 & $\mathrm{G} 130 \mathrm{H}$ & 334 & D & n59119 \\
\hline 24 & 1993 May 12 & $10: 18: 05$ & 9119.93 & $\mathrm{G} 190 \mathrm{H}$ & 334 & & n59120 \\
\hline 24 & 1993 May 12 & $11: 52: 16$ & 9119.99 & $\mathrm{G} 130 \mathrm{H}$ & 334 & $\mathrm{D}$ & $\mathrm{n} 59120$ \\
\hline 25 & 1993 May 13 & $10: 24: 54$ & 9120.93 & $\mathrm{G} 190 \mathrm{H}$ & 334 & & n59121 \\
\hline 25 & 1993 May 13 & $11: 59: 06$ & 9121.00 & $\mathrm{G} 130 \mathrm{H}$ & 334 & & n59121 \\
\hline 26 & 1993 May 14 & $10: 31: 39$ & 9121.93 & $\mathrm{G} 190 \mathrm{H}$ & 334 & & n59122 \\
\hline $\begin{array}{l}26 \\
27\end{array}$ & $\begin{array}{l}1993 \text { May } 14 \\
1993 \text { May } 15\end{array}$ & $\begin{array}{l}12: 05: 50 \\
10: 38: 11\end{array}$ & $\begin{array}{l}9122.00 \\
9122.94\end{array}$ & $\begin{array}{l}\mathrm{G} 130 \mathrm{H} \\
\mathrm{G} 190 \mathrm{H}\end{array}$ & $\begin{array}{l}334 \\
325\end{array}$ & & $\begin{array}{l}\text { n59122 } \\
\text { n59123 }\end{array}$ \\
\hline 27 & 1993 May 15 & $12: 12: 24$ & 9123.00 & $\mathrm{G} 130 \mathrm{H}$ & 325 & & n59123 \\
\hline 28 & 1993 May 16 & $10: 57: 54$ & 9123.95 & $\mathrm{G} 190 \mathrm{H}$ & 325 & & n59124 \\
\hline 28 & 1993 May 16 & $12: 18: 49$ & 9124.01 & $\mathrm{G} 130 \mathrm{H}$ & 325 & & n59124 \\
\hline 29 & 1993 May 17 & $09: 27: 52$ & 9124.89 & $\mathrm{G} 190 \mathrm{H}$ & 325 & & n59125 \\
\hline 29 & 1993 May 17 & $10: 48: 46$ & 9124.95 & $\mathrm{G} 130 \mathrm{H}$ & 325 & & $\mathrm{n} 59125$ \\
\hline 30 & 1993 May 18 & $09: 34: 06$ & 9125.89 & $\mathrm{G} 190 \mathrm{H}$ & 325 & & n59126 \\
\hline 30 & 1993 May 18 & $10: 54: 59$ & 9125.95 & $\mathrm{G} 130 \mathrm{H}$ & 325 & & n59126 \\
\hline 31 & 1993 May 19 & $09: 40: 09$ & 9126.90 & G190H & 325 & & n59127 \\
\hline 31 & 1993 May 19 & $11: 01: 04$ & 9126.96 & $\mathrm{G} 130 \mathrm{H}$ & 325 & & n59127 \\
\hline 32 & 1993 May 20 & $09: 46: 10$ & 9127.90 & $\mathrm{G} 190 \mathrm{H}$ & 324 & & n59128 \\
\hline 32 & 1993 May 20 & $11: 07: 02$ & 9127.96 & $\mathrm{G} 130 \mathrm{H}$ & 324 & & n59128 \\
\hline 33 & 1993 May 21 & $11: 28: 27$ & 9128.98 & G190H & 323 & & n59129 \\
\hline 33 & 1993 May 21 & $12: 49: 20$ & 9129.03 & G130H & 323 & $\mathrm{D}$ & n59129 \\
\hline 34 & 1993 May 22 & $09: 57: 54$ & 9129.91 & $\mathrm{G} 190 \mathrm{H}$ & 322 & $\mathrm{U}$ & $\mathrm{n} 59130$ \\
\hline 34 & 1993 May 22 & $11: 18: 46$ & 9129.97 & $\mathrm{G} 130 \mathrm{H}$ & 322 & $U$ & $\mathrm{n} 59130$ \\
\hline 35 & 1993 May 23 & $10: 03: 42$ & 9130.92 & $\mathrm{G} 190 \mathrm{H}$ & 321 & $\mathrm{U}$ & n59131 \\
\hline 35 & 1993 May 23 & $11: 24: 34$ & 9130.97 & $\mathrm{G} 130 \mathrm{H}$ & 321 & & n59131 \\
\hline 36 & 1993 May 24 & $08: 33: 03$ & 9131.85 & $\mathrm{G} 190 \mathrm{H}$ & 320 & $\mathrm{U}$ & n59132 \\
\hline 36 & 1993 May 24 & $09: 53: 54$ & 9131.91 & $\mathrm{G} 130 \mathrm{H}$ & 320 & & n59132 \\
\hline 37 & 1993 May 25 & $10: 15: 07$ & 9132.93 & $\mathrm{G} 190 \mathrm{H}$ & 319 & & n59133 \\
\hline 37 & 1993 May 25 & $11: 36: 00$ & 9132.98 & $\mathrm{G} 130 \mathrm{H}$ & 319 & & n59133 \\
\hline 38 & 1993 May 26 & $05: 31: 39$ & 9133.73 & $\mathrm{G} 190 \mathrm{H}$ & 318 & & n59134 \\
\hline 38 & 1993 May 26 & $06: 52: 32$ & 9133.79 & $\mathrm{G} 130 \mathrm{H}$ & 318 & & n59134 \\
\hline 39 & 1993 May 27 & $13: 39: 15$ & 9135.07 & $\mathrm{G} 190 \mathrm{H}$ & 317 & & n59135 \\
\hline 39 & 1993 May 27 & $15: 00: 06$ & 9135.12 & $\mathrm{G} 130 \mathrm{H}$ & 317 & & n59135 \\
\hline
\end{tabular}


HST campaign day number, the UT date, the UT time at the start of the exposure, the Julian Date at the start of the exposure, the grating name, the aperture position angle, a comment, and the root names of the spectra for reference by future users (also contained within the IRAF FITS header). The comment codes in column (7) indicate FOS problems we will call "dropouts" (D) and "U-shape anomaly" (U), which we describe in $\$ 2.3 .3$ and Appendix D. The first exposure in each single day (epoch) was the $\mathrm{G} 190 \mathrm{H}$, followed by the $\mathrm{G} 130 \mathrm{H}$. The separation between the two grating exposures in a single day was roughly 80 minutes.

\subsection{The FOS Pipeline Reduction Procedure}

The STScI pipeline reduction system (Post Operations Data Processing System [PODPS]) begins by first converting raw counts to count rates by correcting for quarter-stepping, overscan, and defective diodes. These count rates are then corrected for nonlinearities in the Digicon detectors due to paired pulses (negligible in this case). Background count rates due to Cerenkov radiation from charged particles in the Earth's magnetic field are computed based upon a model that accounts for the dependence on geomagnetic latitude and longitude (day/ night variations) and then subtracted. No observations occurred during passage over the South Atlantic Anomaly. The resulting data are then corrected for small-scale photocathode nonuniformities using appropriate flat fields, and then converted to an absolute flux scale by multiplying the corrected count rates by the appropriate inverse sensitivity curves. The wavelength scales are assigned based upon the template Pt-Cr$\mathrm{Ne}$ spectra obtained during the Science Verification phase of
HST operations. The $\mathrm{G} 190 \mathrm{H}$ and $\mathrm{G} 130 \mathrm{H}$ wavelength scales are essentially linear with small coefficients for the quadratic and cubic terms. For both gratings, wavelength increases with pixel number.

\subsection{The Modified FOS Pipeline}

In practice the PODPS pipeline proved inadequate for the internal accuracy required for this project, and thus modifications of various magnitudes were made in three areas, each of which we will discuss in turn: background subtraction, wavelength calibration, and flux calibration.

\subsubsection{Background Subtraction}

Because of absorption in the FOS magnesium fluoride faceplate, the first 45 or so diodes in a $\mathrm{G} 130 \mathrm{H}$ spectrum have zero sensitivity to first-order photons of wavelength shorter than about $1130 \AA$. It is in this region that one may make a direct measurement of the background count rate; no such region exists for the $\mathrm{G} 190 \mathrm{H}$ spectra. An accurate estimate of the background is very important in the case of the $\mathrm{G} 130 \mathrm{H}$ data, because the detector sensitivity with this grating declines rapidly toward the shorter wavelengths. Figure 2 shows an example of the phenomenon which is present for all $\mathrm{G} 130 \mathrm{H}$ observations: the observed count rate in the zero-sensitivity region lies far above the PODPS model particle background prediction. This count rate in the first $\sim 180$ pixels is very nearly flat, and this was checked for all $\mathrm{G} 130 \mathrm{H}$ observations to confirm that no direct photons (from NGC 5548) were being detected below pixel $\sim 200$. The error in the background estimate, however, does not arise simply from an inaccurate charged-particle

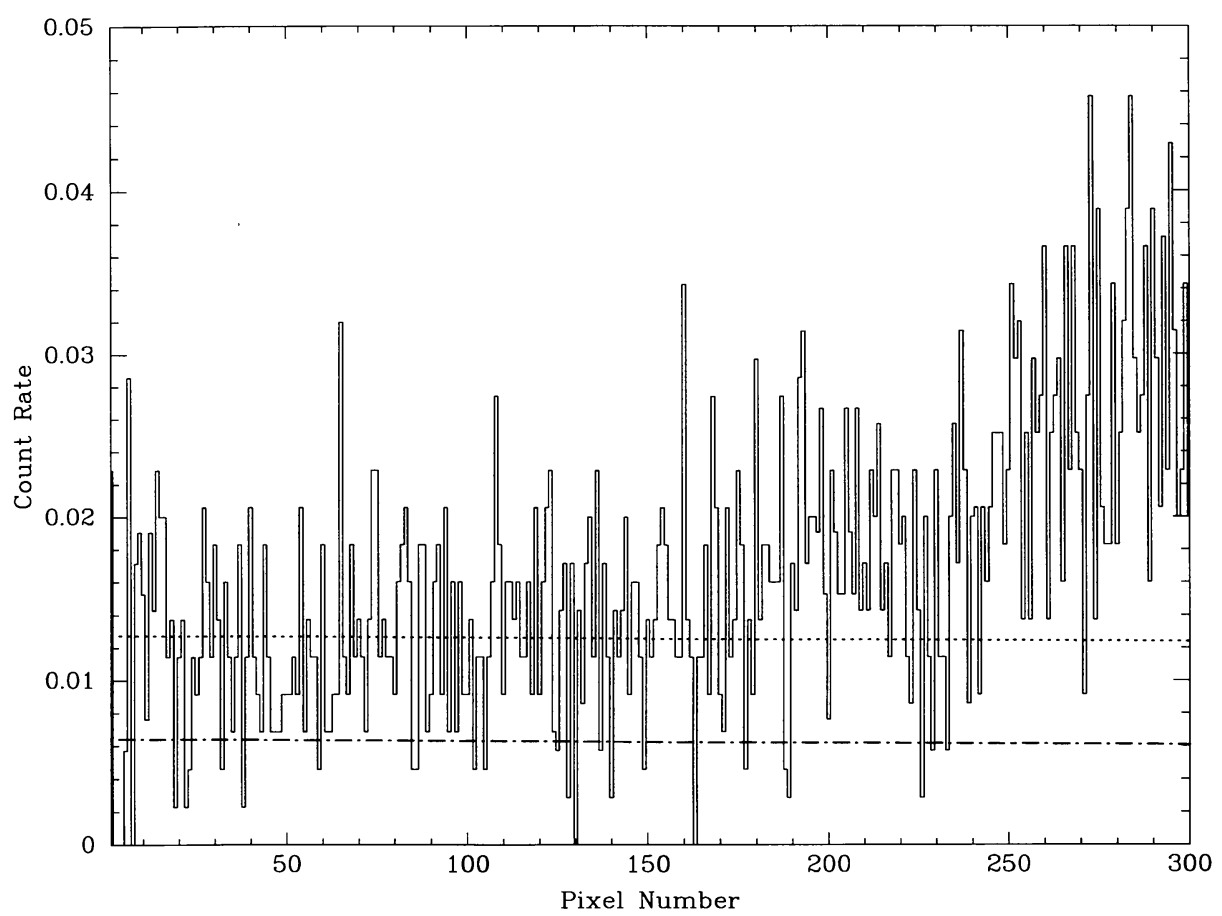

FIG. 2.-Observed count rate vs. pixel number for a $\mathrm{G} 130 \mathrm{H}$ observation. The first $\sim 180$ pixels receive no counts from first-order direct light on account of absorption at the FOS faceplate. This zero-sensitivity region thus allows for a direct measurement of the background level. The dot-dash line shows the PODPS prediction of the charged-particle-induced background level. The large difference between the predicted and observed background count rates was present in all G130H exposures. The dotted line shows the adopted background level. 
background model; for a discussion of the particle background see Appendix A. As we describe in Appendix A, scattered light from near-ultraviolet and optical wavelengths also contributes.

The background correction method employed here is as follows. The mean count rate in a portion of the zero-sensitivity region, pixels $28-128$, was determined for each $\mathrm{G} 130 \mathrm{H}$ spectrum. This mean was determined to a relative accuracy of $\pm 4.4 \%$, based upon counting statistics. Given the uncertainty in the amplitude of the particle background, and the uncertainties in the amplitude and shape of the scattered-light background ( see Appendix A), the following corrections for the two gratings are justified. The $\mathrm{G} 130 \mathrm{H}$ spectra background corrections were applied by assuming that the PODPS particle background rate is correct and then subtracting a constant scattered-light contribution across the diode array, reflecting the offset between the measured background and PODPS-predicted particle background in the zero-sensitivity region of the G130H spectra (see Fig. 2). This is the correction suggested by Kinney \& Bohlin (1993). Since we have no way of directly measuring the background in the $\mathrm{G} 190 \mathrm{H}$ spectra, and since the detector sensitivity is so much greater, no corrections for probable excess background counts over the PODPS-predicted backgrounds were applied to the $\mathrm{G} 190 \mathrm{H}$ data.

\subsubsection{The Intercalibration of the FOS Spectra}

The calibrated wavelength coverage for the two gratings spans roughly the ranges $1155-1605 \AA$ and $1574-2330 \AA$ for the $\mathrm{G} 130 \mathrm{H}$ and $\mathrm{G} 190 \mathrm{H}$, respectively. The relative wavelength calibration, which was performed to bring all the spectra for each grating to the same wavelength scales, was done by crosscorrelating the spectra in the vicinity of the emission-line peaks of $\mathrm{Ly} \alpha+\mathrm{N} \mathrm{V}$ and C IV for the $\mathrm{G} 130 \mathrm{H}$ grating, and around the peaks of He II + O III] and $\mathrm{Si}$ III ] + C III] for the $\mathrm{G} 190 \mathrm{H}$ grating. The cross-correlations were performed on the spectra after subtracting fits to their continua. This was done iteratively in two steps. First, the individual spectra from each grating were crosscorrelated with their respective mean spectra, based upon only those spectra which did not suffer from one or more of the major image misplacement problems described below. The individual spectra were then shifted accordingly, a new mean spectrum was formed, and the process was repeated. The spectra were shifted to the nearest whole pixel, and in most cases the shifts were a single pixel (about $0.25 \AA$ for the $\mathrm{G} 130 \mathrm{H}$ data and about $0.36 \AA$ for the G190H data). Shifts of this amplitude are expected from filter-grating-wheel nonrepeatability. The few spectra which had much larger shifts (2-4 pixels) were known to have been misplaced in the FOS aperture (as explained below). We found that the Galactic absorption lines became significantly sharper in the mean spectrum after shifting the spectra upon the peaks of the strong emission lines. We estimate the uncertainty in this procedure of relative wavelength alignments to be \pm 0.5 pixels. No further shifting in wavelength or scaling in flux was done before joining the $\mathrm{G} 130 \mathrm{H}$ to the $\mathrm{G} 190 \mathrm{H}$ spectrum for every epoch. We discuss this procedure in detail in Appendix B.

\subsubsection{The FOS Flux Calibration and Related Problems}

The absolute ultraviolet flux calibration of the FOS spectra is derived from five spectrophotometric standards BD $+28^{\circ} 4211, \mathrm{BD}+75^{\circ} 325, \mathrm{BD}+33^{\circ} 2642, \mathrm{HZ} 44$, and $\mathrm{WD}$
0501+527 (=G191-B2B). Bohlin et al. (1990) describe the derivation of this absolute flux calibration scale. The internal photometric accuracy of the FOS blue side, based upon repeated observations of well-centered calibration stars, is $\sim 1.4 \%$ (Bohlin 1993a; Lindler \& Bohlin 1994). The uncertainty in the absolute photometric scale arises primarily from the differences between the scale derived above and that from a white dwarf model atmosphere of G191-B2B (Bohlin 1993b). These differences are in the range $5 \%-10 \%$ over the spectral region of interest here. We chose to adopt the latter scale over the former for reasons which will become clear $(\S 5.1)$.

Several fairly well understood photometric problems with the blue-side FOS have been identified. In Appendix $C$ we briefly describe them and their impact upon this data set. The most serious of these problems have been corrected in the NGC 5548 spectra. In addition, the NGC 5548 HST campaign spectra demonstrated three other photometric problems, which we will refer to as (1) the G130H "dropouts," (2) the "U-shape anomaly," and (3) "subgroup variations."

Fortunately, all of the FOS photometric problems and their associated uncertainties had their minimum effects in the spectral region of C IV and He II, the region of primary interest for the present project. Two of the three problems (Nos. 1 and 3) are obvious in Figure 3, which shows the raw light curves for the $\mathrm{G} 190 \mathrm{H}$ and $\mathrm{G130H}$ gratings, in total counts versus Julian Date, for each of the separate subgroup exposures (i.e., separate readouts). Problem 1 affected five $\mathrm{G} 130 \mathrm{H}$ spectra; see the bottom panel of Figure 3. Problem 2 had a $\sim 10 \%$ effect on the spectrophotometry in 10 of the 78 spectra, and do not so easily stand out in Figure 3 except as abrupt 1 day excursions in the light curves. This effect was present in all other spectra at smaller levels. Problem 3 affected most spectra at the $\sim 1 \%$ level, although the "dropouts" were more strongly affected. The magnitudes of all three problems are wavelength-dependent. All are apparently the result of mispointing, either by the spacecraft, by the optical telescope assembly (OTA), within the FOS, or by combinations thereof.

The "dropout" and suspected "U-shape anomaly" observations for which corrections were made are denoted with a $\mathrm{D}$ or a U in column (7) of Table 1. It is important to note that these corrections are relatively uncertain, and that these spectra should be treated with some caution. The corrections were made only to those spectra which were most affected in an attempt to bring them to a similar level of uncertainty as the less "tainted" spectra, i.e., errors of a few percent. The level of photometric uncertainty in the majority of spectra was dominated by the combination of problems 2 and 3 . The level of this uncertainty due to these systematic effects varied from one exposure to the next and is a function of wavelength. It is estimated to range typically from $2 \%$ to $4.5 \%$. We discuss these problems, their effects, and corrections in detail in Appen$\operatorname{dix}$ D.

\subsection{The Combined FOS Spectrum}

In Figure $4 a$ we show a combined $\mathrm{G} 130 \mathrm{H}$ plus $\mathrm{G} 190 \mathrm{H}$ spectrum and its $1 \sigma$ statistical error bar for JD 2,449,105, an average brightness state for the HST campaign. The two spectra were joined where their respective signal-to-noise ratio levels are comparable $(\sim 20)$, and well away from the end of either spectrum. This occurred at about $1595 \AA$, in the observed 

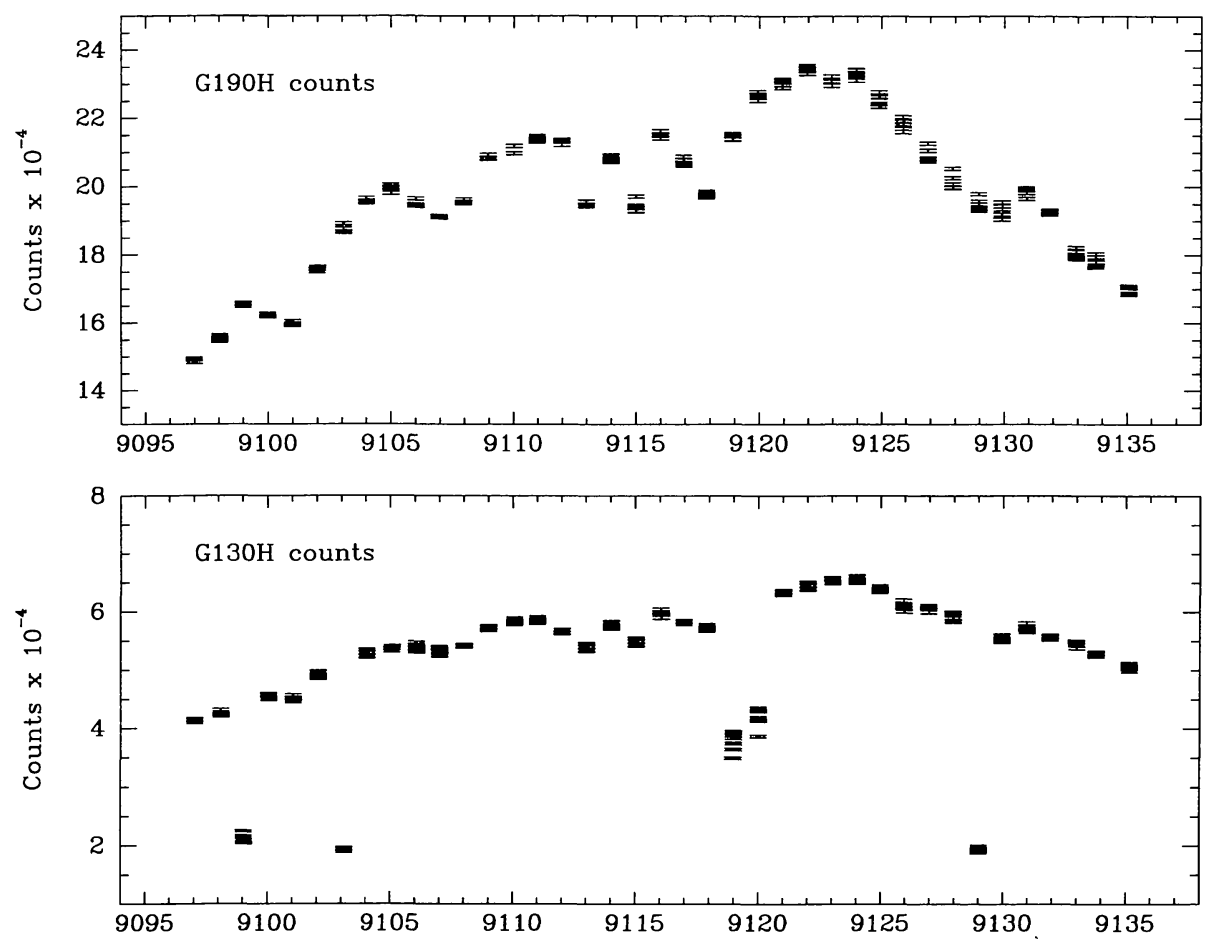

FIG. 3.-Total observed G190H counts (top panel) and G130H counts (bottom panel) summed longward of geocoronal Ly $\alpha$, with their Poisson error bars, vs. Julian Date minus 2,440,000. The subgroups, or separate exposures, for each day's observation are shown individually. Note the 5 days on which the $\mathrm{G} 130 \mathrm{H}$ counts fell drastically.

frame, on the red wing of the C IV emission line. All FOS spectra were joined in this manner; no scale factors were applied, and, as a result, there is a small $(\sim 1 \%)$ discontinuity in flux at the wavelength where the $\mathrm{G} 130 \mathrm{H}$ and $\mathrm{G} 190 \mathrm{H}$ spectra meet. We refer the reader to Appendix B for more details regarding the joining procedure. In Figure $4 b$ we show the combined G130H plus G190H, weighted mean spectrum of NGC 5548 on a log-log plot to highlight the weak features. The spectrum was smoothed lightly by resampling both spectra to the linear wavelength scale appropriate for the $\mathrm{G} 190 \mathrm{H}(0.36725 \AA$ pixel $\left.^{-1}\right)$. The combined spectra which corresponded to the G130H "dropout" exposures were excluded from the construction of this mean spectrum. In addition to the numerous weak emission features, many of which are probably Fe II multiplets, $\sim 20$ weak Galactic absorption lines with equivalent widths ranging over roughly $0.08-0.6 \AA$ are observed. Furthermore, two or three separate systems of absorption intrinsic to NGC 5548 are also observed. Further analyses of the Galactic and other absorption features are deferred to future papers.

\section{THE IUE SPECTRA}

\subsection{The IUE Observations}

The IUE observations were obtained once every 2 days between 1993 March 14 and May 27. These observations began 1 month before and spanned the entire duration of the HST campaign. There were a total of 40 short-wavelength (SWP; 1150-1980 А) camera observations and 35 long-wavelength (LWP; 1950-3300 ̊) camera observations, all of which were taken in the low-resolution mode (5-8 $\AA$ resolution) through the large apertures $\left(10^{\prime \prime} \times 20^{\prime \prime}\right)$. Two exposures failed because of miscentering: SWP 47421 and LWP 25305. The observations were complicated by the presence of scattered solar light in the telescope tube (Weinstein \& Carini 1992), which has been present since 1991 and has become particularly intense at high $\beta$ angles (i.e., the angle between telescope pointing and antisolar direction ). However, since the observations were all obtained at $\beta<55^{\circ}$, the background counts from the fine error sensor (FES; the optical star tracker) were only in the range 50-200 in the slow-track/overlap mode. This background level prevented direct detection of the Seyfert nucleus, so that an optical light curve could not be obtained and the nucleus could not be "locked" to a specific FES coordinate before it was put in an aperture. However, the background was not high enough to cause any problems with the detection and tracking of nearby bright stars. Also, at this level, the contribution of the background to the SWP and LWP spectra is negligible. The nucleus of NGC 5548 was therefore acquired by blind offset from a nearby SAO star, which results in an expected positioning error in the aperture of less than 1". A star in the FES field of view was used for tracking during each exposure, and at each epoch the expected and actual positions of the star were compared to verify that the offset slew was accurate. Logs of the SWP and LWP observations are presented in Tables $2 \mathrm{~A}$ and 2B. The image number, UT date, and time of the beginning of the exposure, the corresponding Julian Date, the exposure time in seconds, and comments are listed in columns (1)-(6), respectively. 


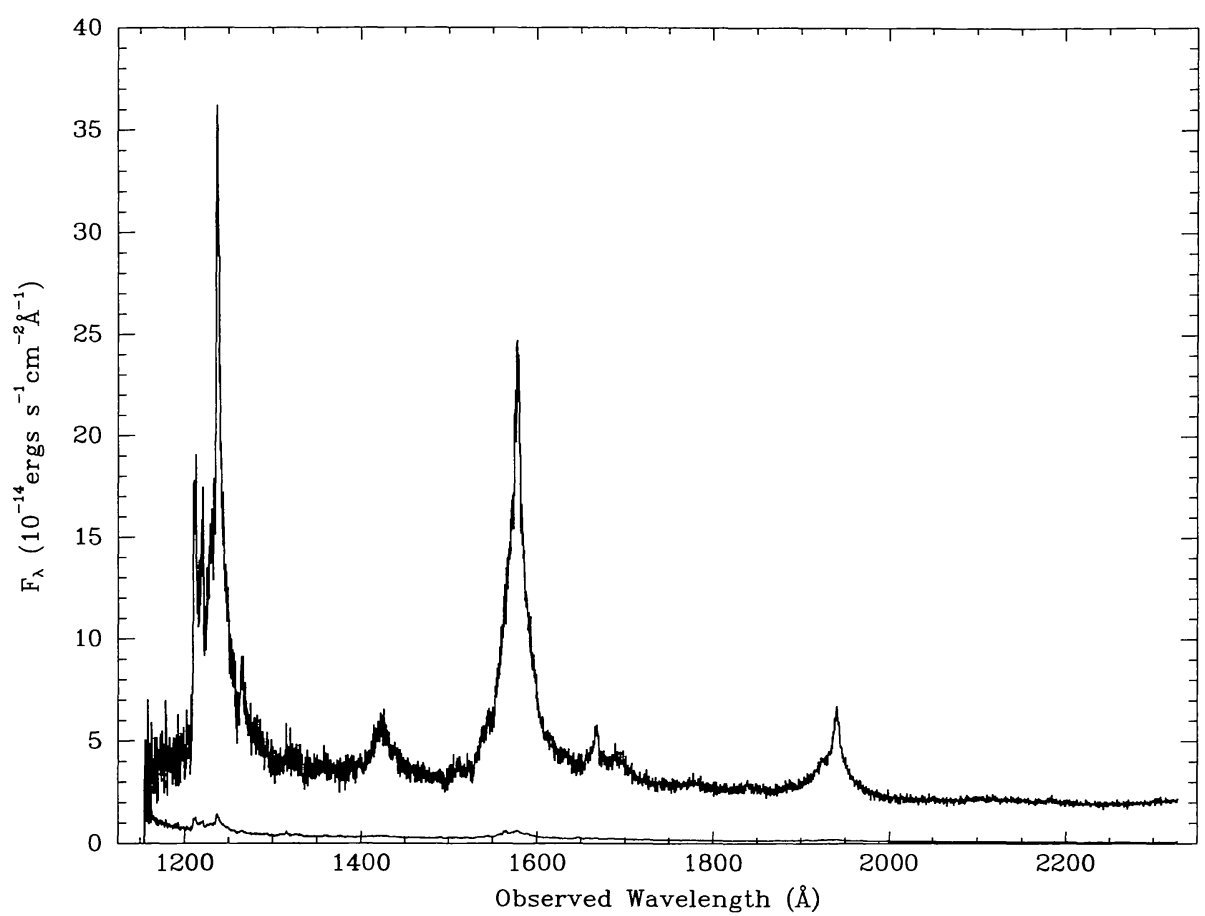

FIG. $4 a$

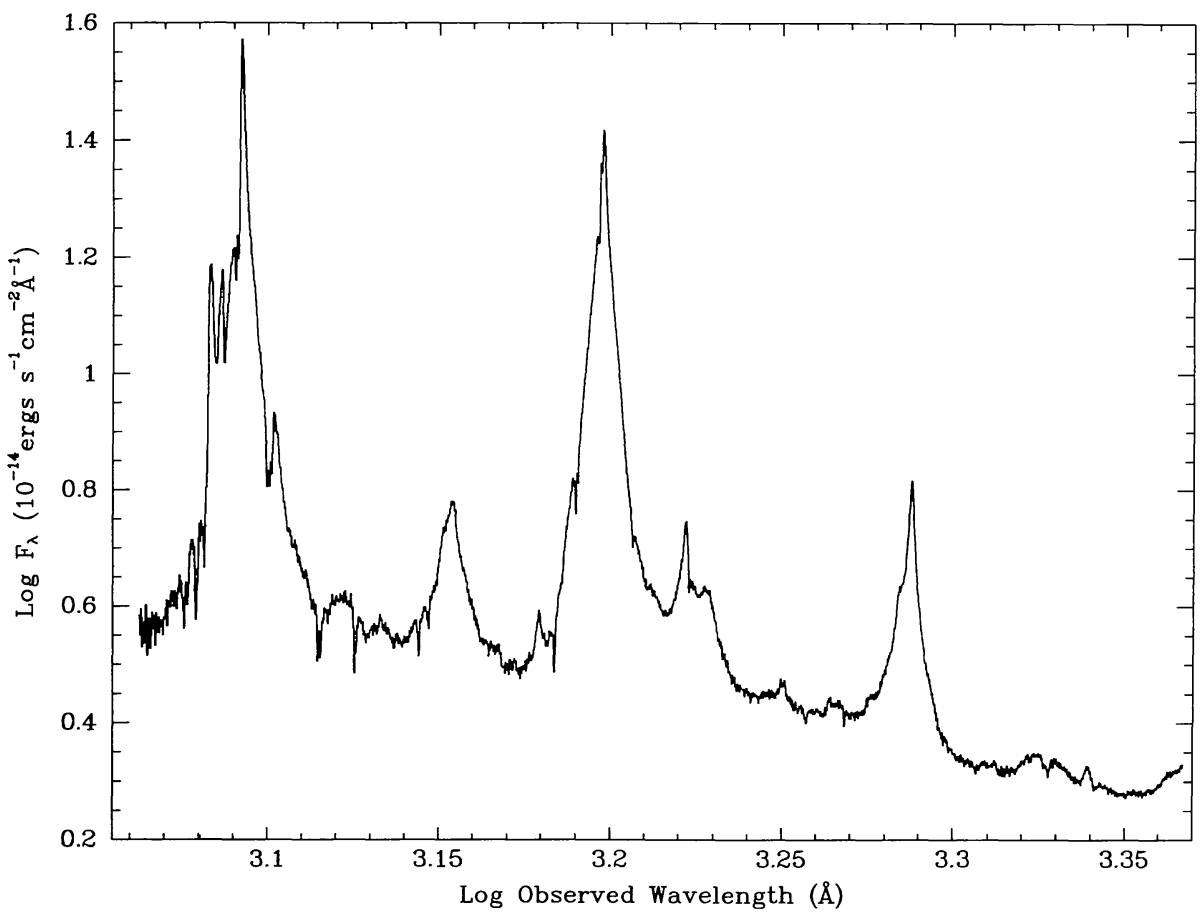

FIG. $4 b$

FIG. 4.- (a) Combined G130H and G190H observed-frame spectrum of NGC 5548 for JD 2,449,105, and its Poisson error bar. Geocoronal Ly $\alpha$ lies on the blue wing of the Ly $\alpha$ broad emission line. Note the many weak emission and absorption features. (b) Combined G130H and G190H weighted-mean spectrum of NGC 5548. The spectrum is plotted in $\log F_{\lambda}$ vs. $\log \lambda$ to highlight the weak emission and absorption features in this high signal-to-noise spectrum. 
TABLE $2 \mathrm{~A}$

LOG OF SWP SPECTROSCOPIC OBSERVATIONS
TABLE 2B

LOG OF LWP SPECTROSCOPIC OBSERVATIONS

\begin{tabular}{|c|c|c|c|c|c|}
\hline \multirow{2}{*}{$\begin{array}{l}\text { Image } \\
\text { Number } \\
\text { (1) }\end{array}$} & \multicolumn{2}{|l|}{ UT } & \multirow{2}{*}{$\begin{array}{c}\text { Julian Date } \\
(2440000+) \\
(4)\end{array}$} & \multirow{2}{*}{$\begin{array}{c}\text { Exposure } \\
\text { Time } \\
(5)\end{array}$} & \multirow[b]{2}{*}{$\begin{array}{c}\text { Comments } \\
(6)\end{array}$} \\
\hline & $\begin{array}{l}\text { Date } \\
(2)\end{array}$ & $\begin{array}{c}\text { Time } \\
(3)\end{array}$ & & & \\
\hline LWP25088 & 1993 Mar 14 & $05: 54: 32$ & 9060.75 & 3600 & \\
\hline LWP25158 & 1993 Mar 20 & $13: 24: 13$ & 9067.06 & 3000 & \\
\hline LWP25174 & 1993 Mar 22 & $13: 23: 22$ & 9069.06 & 3000 & \\
\hline LWP25191 & 1993 Mar 24 & $13: 33: 59$ & 9071.07 & 3600 & header exp. time incorrect \\
\hline LWP25207 & 1993 Mar 26 & $13: 18: 21$ & 9073.05 & 3300 & \\
\hline LWP25223 & 1993 Mar 28 & $13: 01: 53$ & 9075.04 & 3300 & \\
\hline LWP25263 & 1993 Apr 03 & $23: 25: 04$ & 9081.48 & 3600 & \\
\hline LWP25269 & $1993 \mathrm{Apr} 05$ & 03:03:03 & 9082.63 & 3600 & label says LWP25270 \\
\hline LWP25286 & 1993 Арг 07 & $06: 08: 53$ & 9084.76 & 3300 & \\
\hline LPW25305 & 1993 Apr 09 & 04:02:30 & 9086.67 & 3300 & no signal \\
\hline LWP25318 & 1993 Apr 11 & 04:37:59 & 9088.69 & 1320 & weak \\
\hline LWP25337 & 1993 Арr 13 & $03: 42: 40$ & 9090.65 & 3600 & \\
\hline LWP25351 & 1993 Apr 15 & $11: 51: 20$ & 9092.99 & 3600 & \\
\hline LWP25358 & $1993 \mathrm{Apr} 17$ & $12: 09: 27$ & 9095.01 & 3600 & \\
\hline LWP25367 & 1993 Apr 19 & 11:46:05 & 9096.99 & 3600 & \\
\hline LWP25383 & $1993 \mathrm{Apr} 21$ & $11: 41: 02$ & 9098.99 & 3600 & \\
\hline LWP25399 & $1993 \mathrm{Apr} 23$ & $11: 27: 43$ & 9100.98 & 3900 & \\
\hline LWP25409 & 1993 Арг 25 & $11: 45: 33$ & 9102.99 & 3900 & \\
\hline LWP25422 & 1993 Apr 27 & $11: 20: 00$ & 9104.97 & 3900 & \\
\hline LWP25440 & 1993 Apr 29 & $11: 18: 26$ & 9106.97 & 3900 & \\
\hline LWP25452 & 1993 May 01 & 09:51:43 & 9108.91 & 3900 & \\
\hline LWP25464 & 1993 May 03 & $09: 47: 48$ & 9110.91 & 3900 & \\
\hline LWP25472 & 1993 May 05 & 03:06:32 & 9112.63 & 3900 & \\
\hline LWP25483 & 1993 May 07 & 04:07:31 & 9114.67 & 3900 & \\
\hline LWP25496 & 1993 May 09 & $03: 59: 23$ & 9116.67 & 3900 & \\
\hline LWP25514 & 1993 May 10 & 23:53:06 & 9118.50 & 3600 & \\
\hline LWP25522 & 1993 May 13 & $00: 24: 23$ & 9120.52 & 3900 & \\
\hline LWP25531 & 1993 May 15 & $09: 36: 22$ & 9122.90 & 3900 & \\
\hline LWP 25547 & 1993 May 17 & 09:40:43 & 9124.90 & 3900 & \\
\hline LWP25556 & 1993 May 19 & 09:11:04 & 9126.88 & 3900 & \\
\hline LWP25569 & 1993 May 20 & $23: 38: 33$ & 9128.49 & 3900 & \\
\hline LWP25575 & 1993 May 21 & $07: 47: 57$ & 9128.83 & 3900 & \\
\hline LWP25585 & 1993 May 23 & 07:52:04 & 9130.83 & 3600 & \\
\hline LWP25595 & 1993 May 25 & $05: 55: 18$ & 9132.75 & 3120 & \\
\hline LWP25607 & 1993 May 27 & 01:43:59 & 9134.57 & 3900 & \\
\hline
\end{tabular}

ion hit $1420-1520 \AA$ strange He II region

\begin{tabular}{|c|c|c|}
\hline $\begin{array}{c}\text { Julian Date } \\
(2440000+) \\
(4)\end{array}$ & $\begin{array}{c}\text { Exposure } \\
\text { Time } \\
(5)\end{array}$ & $\begin{array}{c}\text { Comments } \\
(6)\end{array}$ \\
\hline 9060.64 & 7200 & \\
\hline 9063.04 & 1680 & very weak spectrum \\
\hline 9065.01 & 3600 & \\
\hline 9067.00 & 4800 & \\
\hline 9069.00 & 4800 & \\
\hline 9071.00 & 4800 & strange He II region \\
\hline 9073.00 & 4800 & ion hit $1460-1472 \AA$ \\
\hline 9074.98 & 4800 & \\
\hline 9077.16 & 3600 & very weak spectrum \\
\hline 9079.32 & 4200 & very weak spectrum \\
\hline 9081.41 & 5400 & \\
\hline 9082.60 & 1920 & no signal; miscentered \\
\hline 9082.68 & 6000 & lines and continuum weak \\
\hline 9084.80 & 5700 & \\
\hline 9086.59 & 6000 & \\
\hline 9088.63 & 5400 & \\
\hline 9090.58 & 6000 & \\
\hline 9092.93 & 4800 & \\
\hline 9094.93 & 4800 & very weak spectrum \\
\hline 9096.93 & 4800 & very weak spectrum \\
\hline 9098.93 & 4800 & \\
\hline 9100.92 & 5100 & \\
\hline 9102.93 & 5100 & \\
\hline 9104.91 & 5100 & \\
\hline 9106.91 & 4800 & strange He II region \\
\hline 9108.84 & 5100 & \\
\hline 9110.84 & 5100 & \\
\hline 9112.56 & 5400 & \\
\hline 9114.72 & 5400 & \\
\hline 9116.72 & 6000 & \\
\hline 9118.55 & 6000 & \\
\hline 9120.57 & 5400 & \\
\hline 9122.84 & 5100 & \\
\hline 9124.84 & 5100 & \\
\hline 9126.82 & 5100 & weak emission lines \\
\hline 9128.53 & 6000 & \\
\hline 9128.87 & 5100 & ion hit $1420-1520 \AA$ \\
\hline 9130.87 & 5100 & strange He II region \\
\hline 9132.68 & 5400 & \\
\hline 9134.50 & 6000 & \\
\hline
\end{tabular}




\subsection{The NEWSIPS Final Archive Processing}

The SWP spectra were processed using the NEWSIPS Final Archive pipeline. This differs from IUESIPS in several important ways. Here we describe those most relevant to the present project (see the CSC-GSFC NEWSIPS publication by Nichols et al. 1993). This method takes advantage of the camera fixed pattern noise to cross-correlate the raw science image with the raw intensity transfer function image. The advantage here is that this fiducial is present even at the lowest light levels. Only one resampling of the data is done, resulting in a geometrically rectified and rotated image whose spectral dispersion function is linear. The spectra are extracted using the signal-weighted extraction technique developed for low-dispersion $I U E$ spectra by Kinney, Bohlin, \& Neill (1991), which is based upon the Horne (1986) optimal extraction technique for long-slit CCD spectrograph data. This technique utilizes information on the cross-dispersion spectral profile, weighting each point in the extraction by its signal-to-noise ratio. An estimated error spectrum is thus extracted, and many, though not all, cosmic-ray hits can be rejected during the extraction. The fluxes are conserved during the extraction. This calibration uses the white dwarf model atmosphere calculations of D. Finley and collaborators, specifically for G191-B2B, to determine the relative sensitivity functions (Bohlin 1993a, b). Finally, the time and temperature (THDA) sensitivity degradation corrections are automatically applied. We note that the NEWSIPS SWP wavelength scale had not been finalized at the time our spectra were extracted.

The LWP spectra were not in line for immediate NEWSIPS processing, and since their measurements were not central to the specific goals of this program, they are deferred to a later paper.

\subsection{Low Signal-to-Noise Ratio Spectra}

A combination of detector sensitivity degradation, SWP exposure times which were generally less than those of the 1988-1989 campaign, a UV nuclear continuum which was on average $\sim 25 \%$ or so fainter than during the $1988-1989$ campaign ( see Paper I), and possibly errors in centering conspired to compromise somewhat the quality of the SWP spectra. The various problems associated with underexposed spectra are apparent in many of the spectra. The errors induced are both wavelength- and intensity-dependent. In two of the worst cases, SWP 47496 and SWP 47505, taken near minimum light of the $I U E$ and $H S T$ campaign, the emission-line fluxes are up to $50 \%$ lower than in an adjacent (in time) better exposed spectrum; the continua are also weak. In SWP 47422 the whole spectrum is at least $50 \%$ weaker than the two adjacent spectra. SWP 47290 and LWP 25318 are extremely weak spectra because their exposure times were far too low. SWP 47387, SWP 47402, SWP 47459, and SWP 47692 were probably also affected. We note that the GEX-extracted versions of the SWP spectra are similarly affected; thus, these photometric problems are not due to errors in the NEWSIPS extractions.

Cosmic-ray hits that were not removed during extraction, sometimes affecting areas several pixels wide, added further noise to the spectra. In the worst case, SWP 47705, an ion hit at a high angle of incidence raised the flux level significantly in a $100 \AA$ band between the Si IV and C IV emission lines.

\section{THE GROUND-BASED OBSERVATIONS}

\subsection{Optical Spectroscopy}

Table 3 is a complete log of spectroscopic observations obtained between 1992 November and 1993 September (hereafter "year 5 "), our fifth observing season of optical monitoring of this galaxy. The format of this table closely follows that of Papers II, III, and VII; the most significant change is that dates and times of observation are given to greater precision than previously because of the shorter timescales that will be examined in this experiment. Columns ( 1 ) and (2) give the UT date and time at the beginning of each observation, and column (3) shows the corresponding Julian Date. Column (4) gives a code which indicates the observatory and instrument used to obtain the spectrum; these codes are the same as in Papers II, III, IV, and VII of this series, whenever possible. The projected spectrograph entrance aperture, in arcseconds, is given in column (5). The first parameter is the slit width in the dispersion direction, and the second parameter is the slit length in the crossdispersion direction (i.e., the "extraction window" for two-dimensional detectors). The slit position angle is given in column (6), measured in the conventional manner, in degrees eastward from north; the cross-dispersion direction runs northsouth for a position angle $0^{\circ}$. An estimate of the seeing, when it was recorded at the telescope, is given in column (7). In the case of the Ohio State spectra (set A), the value given is the FWHM of the broad component of the $\mathrm{H} \beta$ emission line measured in the cross-dispersion direction; since the BLR is spatially unresolved, this provides a good description of the PSF that characterizes both atmospheric seeing and tracking variations. The nominal spectral resolution is given in column (8), and the approximate wavelength range covered by the data is given in column (9). Finally, to aid archival use of these data, column (10) gives a unique identifier by which the spectrum is known to the IRAF reduction system and which is contained in the FITS file header. The file naming convention is the same as used in previous papers: the first two characters ( 15 ) identify the galaxy as NGC 5548, and the next four characters (e.g., 8954 ) contain the four least significant figures in the truncated Julian Date, as in column (3). The next character gives the observatory code, as in column (4). An additional arbitrary character is added when necessary to eliminate any remaining ambiguity.

\subsection{Optical Photometry}

Optical broadband flux measurements, based on either CCD imaging or photoelectric aperture photometry, were made on several occasions with a number of telescopes. In order to make the data as homogeneous as possible, most observations were made in Johnson $V$ through circular apertures of projected radius 8 ".0. Nevertheless, primarily because of slight differences, for example in various filter bandpasses and detector response functions, we find that there are some systematic differences among the sets of data, and we will discuss how these are accounted for in the next section. In general, the absolute calibration of the photometry is tied to star 1 of Penston, Penston, \& Sandage (1971), which has been recalibrated as part of this project by Romanishin et al. (1994); we adopt the recalibrated magnitudes for this star, namely, $V=13.75, B-$ 
TABLE 3

LOG OF SPECTROSCOPIC OBSERVATIONS

\begin{tabular}{|c|c|c|c|c|c|c|c|c|c|c|c|c|c|c|c|c|c|c|c|}
\hline \multicolumn{2}{|l|}{ UT } & \multirow{2}{*}{$\begin{array}{c}\text { Julian Date } \\
(2440000+) \\
(3)\end{array}$} & \multirow[b]{2}{*}{$\begin{array}{l}\text { Code } \\
\text { (4) }\end{array}$} & \multicolumn{2}{|c|}{ Aperture } & \multirow{2}{*}{$\begin{array}{c}\text { Seeing } \\
(") \\
(7)\end{array}$} & \multirow{2}{*}{$\begin{array}{l}\text { Res. } \\
(\AA) \\
(8)\end{array}$} & \multirow{2}{*}{$\begin{array}{c}\text { Range } \\
(\AA) \\
(9)\end{array}$} & \multirow{2}{*}{$\begin{array}{c}\text { IRAF } \\
\text { file name } \\
\text { (10) }\end{array}$} & \multicolumn{2}{|l|}{ UT } & \multirow{2}{*}{$\begin{array}{c}\text { Julian Date } \\
(2440000+) \\
(3)\end{array}$} & \multirow[b]{2}{*}{$\begin{array}{l}\text { Code } \\
\text { (4) }\end{array}$} & \multicolumn{2}{|c|}{ Aperture } & \multirow{2}{*}{$\begin{array}{c}\text { Seeing } \\
(") \\
(7)\end{array}$} & \multirow{2}{*}{$\begin{array}{c}\text { Res. } \\
(\AA) \\
(8)\end{array}$} & \multirow{2}{*}{$\begin{array}{c}\text { Range } \\
(\AA) \\
(9)\end{array}$} & IRAF \\
\hline $\begin{array}{c}\text { Date } \\
(1)\end{array}$ & $\begin{array}{c}\text { Time } \\
(2)\end{array}$ & & & $\begin{array}{l}\text { Size } \\
(5) \\
\end{array}$ & $\begin{array}{l}\text { P.A. } \\
(6)\end{array}$ & & & & & $\begin{array}{c}\text { Date } \\
(1)\end{array}$ & $\begin{array}{c}\text { Time } \\
(2)\end{array}$ & & & $\begin{array}{l}\text { Size } \\
(5) \\
\end{array}$ & $\begin{array}{l}\text { P.A. } \\
(6)\end{array}$ & & & & $\begin{array}{c}\text { file name } \\
(10)\end{array}$ \\
\hline 1992 Nov 27 & $12: 41$ & 8954.03 & A & $5.0 \times 7.5$ & 90 & 5.0 & 9 & $4550-5710$ & n58954a & 1993 Mar 25 & $11: 20$ & 9071.98 & $\mathrm{~F}$ & $3.2 \times 6.4$ & 90 & & 5 & $4720-7150$ & n59071fb \\
\hline 1992 Dec 10 & $12: 00$ & 8967.00 & A & $5.0 \times 7.5$ & 90 & 4.4 & 9 & $4520-5680$ & n58967a & 1993 Mar 28 & $00: 40$ & 9074.53 & $\mathrm{~W}$ & $3.0 \times 10.0$ & 90 & 5 & 9 & $4500=5630$ & $\mathrm{n} 59074 \mathrm{w}$ \\
\hline 1992 Dec 16 & $12: 19$ & 8973.01 & A & $5.0 \times 7.5$ & 90 & 4.2 & 9 & $4510-5670$ & n58973a & 1993 Mar 28 & 12:06 & 9075.01 & $\mathrm{~F}$ & $3.2 \times 6.4$ & 90 & & 5 & $4720-7150$ & n59075f \\
\hline 1992 Dec 24 & $12: 47$ & 8981.03 & A & $5.0 \times 7.5$ & 90 & 3.2 & 9 & $4500-5660$ & n58981a & 1993 Mar 30 & $21: 30$ & 9077.39 & $\mathrm{~W}$ & $3.0 \times 10.0$ & 90 & 3 & 9 & $4390-5590$ & n59077w \\
\hline 1993 Dec 25 & $13: 16$ & 8982.05 & $\mathrm{~F}$ & $3.2 \times 6.4$ & 90 & & 5 & $4670-7160$ & n58982f & 1993 Mar 31 & $21: 00$ & 9078.41 & W & $3.0 \times 10.0$ & 90 & 3 & 9 & $4450-5630$ & n59078w \\
\hline 1993 Jan 4 & $12: 16$ & 8992.01 & A & $5.0 \times 7.5$ & 90 & 3.1 & 9 & $4540-5700$ & n58992a & 1993 Mar 31 & $22: 34$ & 9078.44 & M & $3.0 \times 10.0$ & 0 & 2.5 & 5 & $4430-7130$ & n59078m \\
\hline 1993 Jan 12 & $12: 29$ & 9000.02 & A & $5.0 \times 7.5$ & 90 & 3.1 & 9 & $4650-5670$ & n59000a & 1993 Apr 2 & $08: 20$ & 9079.85 & A & $5.0 \times 7.5$ & 90 & 4.4 & 9 & $4500-5670$ & n59079a \\
\hline $1993 \operatorname{Jan} 17$ & 03:32 & 9004.65 & B & $15.0 \times 11.0$ & 3.6 & $2-3$ & 15 & $4510-7910$ & n59004b & 1993 Apr 2 & $23: 10$ & 9080.46 & M & $3.0 \times 10.0$ & 0 & 3 & 5 & $4420-7120$ & n59080m \\
\hline 1993 Jan 21 & $11: 42$ & 9008.99 & A & $5.0 \times 7.5$ & 90 & 3.1 & 9 & $4510-5680$ & n59008a & 1993 Apr 5 & $22: 58$ & 9083.46 & M & $3.0 \times 10.0$ & 0 & 2 & 5 & $4470-7170$ & n59083m \\
\hline 1993 Jan 22 & $12: 58$ & 9010.04 & $\mathrm{~F}$ & $3.2 \times 6.4$ & 90 & & 5 & $4660-7140$ & n59010f & 1993 Apr 7 & $17: 14$ & 9085.22 & $\mathrm{Y}$ & $4.0 \times 11.5$ & 90 & $3-4$ & 11 & $4300-7000$ & n59085y \\
\hline $1993 \mathrm{Jan} 23$ & $01: 25$ & 9010.56 & W & $3.0 \times 10.0$ & 90 & 3 & 9 & $4350-5550$ & n59010wa & 1993 Apr 8 & $07: 38$ & 9085.82 & A & $5.0 \times 7.5$ & 90 & 3.0 & 9 & $4510-5670$ & n59085a \\
\hline 1993 Jan 23 & 03:10 & 9010.63 & $\mathrm{~W}$ & $3.0 \times 10.0$ & 90 & 3 & 9 & $6120-7240$ & n59010wb & 1993 Apr 9 & $16: 53$ & 9087.20 & $\mathrm{Y}$ & $4.0 \times 11.5$ & 90 & $3-4$ & 11 & $4300-7000$ & n59087y \\
\hline $1993 \mathrm{Jan} 23$ & $12: 48$ & 9011.03 & $\mathrm{~F}$ & $3.2 \times 6.4$ & 90 & & 5 & $4660-7140$ & n59011f & 1993 Apr 10 & $16: 43$ & 9088.20 & $\mathrm{Y}$ & $4.0 \times 11.5$ & 90 & $2-3$ & 11 & $4300-7000$ & n59088y \\
\hline 1993 Jan 24 & $12: 45$ & 9012.03 & $\mathrm{~F}$ & $3.2 \times 6.4$ & 90 & & 5 & $4660-7140$ & n59012f & 1993 Apr 10 & $19: 35$ & 9088.32 & $\mathrm{~W}$ & $3.0 \times 10.0$ & 90 & 3 & 9 & $4500-5540$ & n59088wa \\
\hline 1993 Jan 25 & $03: 24$ & 9012.64 & B & $15.0 \times 11.0$ & 3.6 & $2-3$ & 15 & $4510-7910$ & n59012b & 1993 Apr 10 & $21: 45$ & 9088.41 & W & $3.0 \times 10.0$ & 90 & 3 & 9 & $6180-7200$ & n59088wb \\
\hline 1993 Jan 25 & $12: 48$ & 9013.03 & $\mathrm{~F}$ & $3.2 \times 6.4$ & 90 & & 5 & $4660-7140$ & n59013f & $1993 \mathrm{Apr} 11$ & $16: 05$ & 9089.17 & $\mathrm{Y}$ & $4.0 \times 11.5$ & 90 & $2-3$ & 11 & $4300-7000$ & n59089y \\
\hline $1993 \operatorname{Jan} 26$ & $06: 40$ & 9013.78 & $\mathrm{~T}$ & $1.4 \times 17.7$ & 160 & 3 & 2.5 & $6218-7131$ & n59013t & $1993 \mathrm{Apr} 11$ & $21: 40$ & 9089.40 & $\mathrm{~W}$ & $3.0 \times 10.0$ & 90 & 3 & 9 & $4400-5570$ & n59089w \\
\hline 1993 Jan 26 & $10: 30$ & 9013.94 & A & $50 \times 7.5$ & 90 & 2.8 & 9 & $4540-5700$ & n59013a & $1993 \mathrm{Apr} 12$ & $16: 07$ & 9090.17 & $\mathrm{Y}$ & $4.0 \times 11.5$ & 90 & 2 & 11 & $4300-7000$ & n59090y \\
\hline 1993 Jan 26 & $13: 09$ & 9014.05 & $\mathrm{~F}$ & $3.2 \times 6.4$ & 90 & & 5 & $4660-7140$ & n59014f & $1993 \mathrm{Apr} 13$ & $07: 37$ & 9090.82 & $\mathrm{~F}$ & $3.2 \times 6.4$ & 90 & & 5 & $4720-7150$ & n59090f \\
\hline 1993 Jan 27 & $00: 20$ & 9014.51 & $\mathrm{~W}$ & $3.0 \times 10.0$ & 90 & 3 & 0 & $4370-5550$ & n59014wa & $1993 \mathrm{Apr} 13$ & $08: 20$ & 909085 & A & $5.0 \times 7.5$ & 90 & 5.2 & 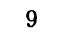 & $4510-5680$ & n59090a \\
\hline 1993 Jan 27 & $02: 10$ & 9014.59 & W & $3.0 \times 10.0$ & 90 & 2 & 9 & $6070-7200$ & n59014wb & 1993 Apr 13 & $10: 09$ & 9090.92 & $\mathrm{H}$ & $4.0 \times 10.0$ & 51 & 1 & 8 & $3140-8038$ & n59090h \\
\hline 1993 Jan 29 & $06: 46$ & 16.78 & $\mathrm{~T}$ & $1.3 \times 4.8$ & 160 & 2 & 2.5 & $6158-7071$ & n59016t & $1993 \mathrm{Apr} 13$ & $15: 41$ & 909115 & $\mathrm{Y}$ & $4.0 \times 11.5$ & 90 & 2 & 11 & $4300-7000$ & n59091y \\
\hline 1993 Jan 29 & $13: 58$ & 9017.08 & $\mathrm{H}$ & $4.0 \times 10.0$ & 0 & 3 & 8 & $3262-10500$ & n59017ha & $1993 \mathrm{Apr} 14$ & $08: 26$ & 9091.86 & $\mathrm{~F}$ & $3.2 \times 6.4$ & 90 & & 5 & $4720-7150$ & n59091f \\
\hline $1993 \operatorname{Jan} 29$ & $14: 11$ & 9017.09 & $\mathrm{H}$ & $4.0 \times 10.0$ & 0 & 3 & 4 & $5830-7100$ & $\mathrm{n} 59017 \mathrm{hb}$ & $1993 \mathrm{Apr} 14$ & $11: 37$ & 98 & $\mathrm{H}$ & $4.0 \times 10.0$ & 61 & 1 & 8 & -8040 & n59091h \\
\hline $1993 \mathrm{Feb} 2$ & $11: 11$ & 9020.97 & A & $5.0 \times 7.5$ & 90 & 4.3 & 9 & $4520-5690$ & $\mathrm{n} 59020 \mathrm{a}$ & $1993 \mathrm{Apr} 15$ & $08: 37$ & 9092.86 & $\mathrm{H}$ & $4.0 \times 10.0$ & 146 & 2 & 8 & $3120-8040$ & n59092h \\
\hline 1993 Feb 11 & $10: 55$ & 9029.95 & A & $5.0 \times 7.5$ & 90 & 4.3 & 9 & $4540-5680$ & n59029a & $1993 \mathrm{Apr} 15$ & $08: 40$ & 9092.86 & $\mathrm{~F}$ & $3.2 \times 6.4$ & 90 & & 5 & -7150 & n59092f \\
\hline $1993 \mathrm{Feb} 13$ & $00: 58$ & 54 & $\mathrm{R}$ & $1.5 \times 12.5$ & 0 & & 10 & $4540-7030$ & $\mathrm{~m}$ & 1993 Apr 16 & $18: 04$ & 094 & $\mathrm{Y}$ & $4.0 \times 11.5$ & 90 & 4 & 11 & -7000 & n59094y \\
\hline $1993 \mathrm{Feb} 13$ & $13: 52$ & 9032.08 & $\mathrm{H}$ & $4.0 \times 10.0$ & 44 & $3-4$ & 8 & $3090-8056$ & $\mathrm{n} 59032 \mathrm{~h}$ & $1993 \mathrm{Apr} 17$ & $01: 38$ & 9094.57 & B & $15.0 \times 11.0$ & 3.6 & $2-3$ & 15 & $4510-7910$ & n59094b \\
\hline 1993 Feb 16 & $00: 10$ & 9 & W & 100 & 90 & 9 & 0 & -5520 & & $1993 \mathrm{Apr} 17$ & $11: 46$ & 90 & $\mathrm{~F}$ & $3.2 \times 6.4$ & 90 & & 5 & -7150 & \\
\hline $1993 \mathrm{Feb} 16$ & $02: 25$ & 9034.60 & W & $3.0 \times 10.0$ & 90 & 9 & 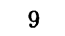 & $6050-7180$ & n59034wb & $1993 \mathrm{Apr} 17$ & $17: 31$ & 23 & $\mathrm{Y}$ & $4.0 \times 11.5$ & 90 & 2 & 11 & -7000 & n59095y \\
\hline $1993 \mathrm{Feb} 17$ & $12: 00$ & 9 & $\mathrm{~F}$ & 64 & 90 & & 5 & 466 & & Apr 19 & $11: 38$ & & $\mathrm{~F}$ & $32 \times 64$ & 90 & & 5 & 7150 & $96 f$ \\
\hline $1993 \mathrm{Feb} 21$ & $01: 13$ & 9039.55 & $\mathrm{~L}$ & 3.0 round & - & 3 & 3 & $4240-5262$ & n59039l & 1993 Apr 21 & $07: 36$ & 9098.82 & $\mathrm{~F}$ & $3.2 \times 6.4$ & 90 & & 5 & $4720-7150$ & n59098f \\
\hline $1993 \mathrm{Feb} 21$ & $23: 58$ & 9040.50 & $\mathrm{~L}$ & 3.0 round & - & 4 & 3 & $=5262$ & & 3 Apr 21 & $07: 06$ & & $\mathrm{Z}$ & $2.0 \times 10.0$ & 0 & 2.5 & 5 & -5900 & n59098za \\
\hline $1993 \mathrm{Feb} 26$ & 13:09 & 9045.04 & $\mathrm{~F}$ & $3.2 \times 6.4$ & 90 & & 5 & $4660-7140$ & $\mathrm{n} 59045 \mathrm{fa}$ & 1993 Apr 21 & $07: 36$ & 9098.82 & $\mathrm{Z}$ & $2.0 \times 10.0$ & 0 & 2.5 & 5 & $5850-6960$ & n59098zb \\
\hline $1993 \mathrm{Feb} 27$ & $11: 03$ & 1500 & F & $3.2 \times 6.4$ & 90 & & 5 & $4660-7140$ & & 1993 Apr 22 & $07: 11$ & & A & $5.0 \times 7.5$ & 90 & 2.1 & 9 & $4570-5720$ & n59099a \\
\hline 1993 Mar 2 & $10: 36$ & 9048.94 & A & $5.0 \times 7.5$ & 90 & 3.9 & 9 & $4530-5700$ & $\mathrm{n} 590$ & $1993 \mathrm{Apr} 23$ & $16: 41$ & 9101.19 & $\mathrm{Y}$ & $4.0 \times 11.5$ & 90 & $3-4$ & 11 & $4300-7000$ & $\mathrm{n} 59101 \mathrm{y}$ \\
\hline 1993 Mar 10 & $09: 32$ & & A & $5.0 \times 7.5$ & 90 & 4.5 & 0 & 5600 & & Apr 23 & $22: 09$ & & B & & 3.6 & $2-3$ & 15 & & n59101b \\
\hline 1993 Mar 15 & $09: 33$ & 9 & $\mathrm{~F}$ & $3.2 \times 6.4$ & 90 & & 5 & 0 & & 1993 Apr 24 & $16: 50$ & & $\mathrm{Y}$ & $\mathrm{x} 11.5$ & 90 & $3-4$ & 11 & 000 & $02 \mathrm{y}$ \\
\hline $1993 \mathrm{Mar} 16$ & $08: 56$ & 9062 & A & $5.0 \times 7.5$ & 90 & 3. & 9 & & & $1993 \mathrm{Apr} 25$ & $11: 32$ & & $\mathrm{~F}$ & $3.2 \times 6.4$ & 90 & & 5 & -7150 & $\mathrm{n} 59102 \mathrm{f}$ \\
\hline 1993 Mar 18 & $07: 16$ & 9064.80 & A & $5.0 \times 7.5$ & 90 & 3.5 & 9 & 5680 & $64 \mathrm{a}$ & 1993 Apr 26 & $03: 21$ & 9103.64 & $\mathrm{Z}$ & $2.0 \times 10.0$ & 0 & 3.0 & 5 & 5900 & n59103za \\
\hline 1993 Mar 18 & $12: 11$ & 65.01 & $\mathrm{~F}$ & $3.2 \times 6.4$ & 90 & & 5 & $4660-7140$ & n59065f & 1993 Apr 26 & $03: 59$ & & $\mathrm{Z}$ & $2.0 \times 10.0$ & 0 & 0.0 & 5 & $5850-6960$ & n59103zb \\
\hline 1993 Mar 19 & $12: 19$ & 9066.02 & $\mathrm{~F}$ & $3.2 \times 6.4$ & 90 & & 5 & $4720-7150$ & & 1993 Apr 29 & $05: 24$ & & $\mathrm{Z}$ & $2.0 \times 10.0$ & 0 & 3.0 & 5 & $4620-5900$ & n59106za \\
\hline $1993 \mathrm{Mar} 20$ & $12: 26$ & 9067.02 & $\mathrm{~F}$ & $3.2 \times 6.4$ & 90 & & 5 & $4720-7150$ & n59067f & 1993 Apr 29 & $05: 51$ & & $\mathrm{Z}$ & $2.0 \times 10.0$ & 0 & 3.0 & 5 & & n59106zb \\
\hline 1993 Mar 23 & 11: & & $\mathrm{F}$ & & 90 & & 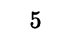 & & & Apr 29 & & & $\mathrm{U}$ & 6.0 & 90 & & 7 & 9950 & $\mathrm{n} 59107 \mathrm{u}$ \\
\hline 1993 Mar 23 & $23: 05$ & 9070.46 & W & $3.0 \times 10.0$ & 90 & 2 & 9 & $4370-5560$ & n59070wa & 1993 Apr 30 & $07: 40$ & 9107.82 & A & $5.0 \times 7.5$ & 90 & 4.5 & 9 & $4510-5660$ & n59107a \\
\hline 1993 Mar 24 & $00: 40$ & 9070.53 & W & $3.0 \times 10.0$ & 90 & 2 & 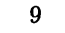 & -1200 & $\mathrm{n} 5$ & 1993 Apr 30 & $11: 36$ & & 1 & $4.0 \times 10.0$ & 61 & $2-3$ & 8 & $3114-8032$ & n59107h \\
\hline 1993 Mar 24 & $12: 02$ & 9071.00 & $\mathrm{~F}$ & $3.2 \times 6.4$ & 90 & & 5 & $4720-7150$ & n59071fa & 1993 May 4 & $17: 42$ & 9112.24 & $\mathrm{Y}$ & $4.0 \times 11.5$ & 90 & 3 & 11 & $4300-7000$ & n59112y \\
\hline
\end{tabular}


TABLE 3-Continued

\begin{tabular}{|c|c|c|c|c|c|c|c|c|c|c|c|c|c|c|c|c|c|c|c|}
\hline \multicolumn{2}{|l|}{ UT } & \multirow{2}{*}{$\begin{array}{c}\text { Julian Date } \\
(2440000+) \\
(3)\end{array}$} & \multirow[b]{2}{*}{$\begin{array}{l}\text { Code } \\
(4)\end{array}$} & \multicolumn{2}{|c|}{ Aperture } & \multirow{2}{*}{$\begin{array}{c}\text { Seeing } \\
(") \\
(7)\end{array}$} & \multirow{2}{*}{$\begin{array}{c}\text { Res. } \\
(\AA) \\
(8)\end{array}$} & \multirow{2}{*}{$\begin{array}{c}\text { Range } \\
(\AA) \\
(9)\end{array}$} & \multirow{2}{*}{$\begin{array}{c}\text { IRAF } \\
\text { file name } \\
(10)\end{array}$} & \multicolumn{2}{|l|}{ UT } & \multirow{2}{*}{$\begin{array}{c}\text { Julian Date } \\
(2440000+) \\
(3)\end{array}$} & \multirow[b]{2}{*}{$\begin{array}{c}\text { Code } \\
\text { (4) }\end{array}$} & \multicolumn{2}{|c|}{ Aperture } & \multirow{2}{*}{$\begin{array}{c}\text { Seeing } \\
\left({ }^{\prime \prime}\right) \\
(7)\end{array}$} & \multirow{2}{*}{$\begin{array}{l}\text { Res. } \\
(\AA) \\
(8)\end{array}$} & \multirow{2}{*}{$\begin{array}{c}\text { Range } \\
(\AA) \\
(9)\end{array}$} & IRAF \\
\hline $\begin{array}{l}\text { Date } \\
\text { (1) }\end{array}$ & $\begin{array}{l}\text { Time } \\
(2)\end{array}$ & & & $\begin{array}{c}\text { Size } \\
(5)\end{array}$ & $\begin{array}{l}\text { P.A. } \\
(6)\end{array}$ & & & & & $\begin{array}{l}\text { Date } \\
\text { (1) }\end{array}$ & $\begin{array}{l}\text { Time } \\
(2)\end{array}$ & & & $\begin{array}{l}\text { Size } \\
(5)\end{array}$ & $\begin{array}{l}\text { P.A. } \\
\text { (6) }\end{array}$ & & & & $\begin{array}{l}\text { file name } \\
\text { (10) }\end{array}$ \\
\hline 1993 May 5 & $17: 47$ & 9113.24 & $\mathrm{Y}$ & $4.0 \times 11.5$ & 90 & 3 & 11 & $4300-7000$ & n59113y & \begin{tabular}{|l|}
1993 Jun 21 \\
\end{tabular} & $04: 32$ & 9159.69 & $F$ & $3.2 \times 6.4$ & 90 & & 5 & $4720-7170$ & n59159f \\
\hline 1993 May 6 & $22: 04$ & 9114.42 & M & $3.0 \times 10.0$ & 0 & 2 & 5 & $4950-7440$ & n59114mb & 1993 Jun 22 & 03:53 & 9160.66 & F & $3.2 \times 6.4$ & 90 & & 5 & $4660-7170$ & n59160f \\
\hline 1993 May 7 & $00: 14$ & 9114.51 & M & $3.0 \times 10.0$ & 0 & 2.5 & 5 & $4740-7440$ & n59114ma & 1993 Jun 25 & 04:29 & 9163.69 & A & $5.0 \times 7.5$ & 90 & 3.5 & 9 & $4530-5680$ & n59163a \\
\hline 1993 May 7 & $07: 25$ & 9114.81 & A & $5.0 \times 7.5$ & 90 & 3.1 & 9 & $4470-5630$ & n59114a & 1993 Jun 25 & $20: 57$ & 9164.37 & B & $15.0 \times 11.0$ & 3.6 & $2-3$ & 15 & $4510-7910$ & n59164b \\
\hline 1993 May 13 & $05: 52$ & 9120.74 & $\mathrm{~J}$ & $2.1 \times 10.0$ & 90 & 1.5 & 5 & $4570-7260$ & $\mathrm{n} 59120 \mathrm{j}$ & 1993 Jun 28 & $08: 13$ & 9166.84 & $\mathrm{H}$ & $4.0 \times 10.0$ & 61 & 1.5 & 8 & $3110-8040$ & n59166h \\
\hline 1993 May 13 & $07: 17$ & 9120.81 & F & $3.2 \times 6.4$ & 90 & & 5 & $4720-7150$ & n59120f & $1993 \mathrm{Jul} 1$ & $04: 31$ & 9169.69 & A & $5.0 \times 7.5$ & 90 & 3.1 & 9 & $4530-5680$ & n59169a \\
\hline 1993 May 14 & $06: 35$ & 9121.77 & $J$ & $2.1 \times 10.0$ & 90 & 1.5 & 5 & $4570-7260$ & n59122j & $1993 \mathrm{Jul} 8$ & $04: 28$ & 9176.69 & A & $5.0 \times 7.5$ & 90 & 3.1 & 9 & $4530-5690$ & n59176a \\
\hline 1993 May 15 & $08: 10$ & 9122.84 & A & $5.0 \times 7.5$ & 90 & 1.5 & 9 & $4530-5600$ & n59122a & $1993 \mathrm{Jul} 14$ & 08:19 & 9182.85 & $\mathrm{H}$ & $4.0 \times 10.0$ & 57 & 2.0 & 8 & $3112-8034$ & n59182h \\
\hline 1993 May 18 & $03: 48$ & 9125.66 & $\mathrm{~F}$ & $3.2 \times 6.4$ & 90 & & 5 & $4660-7160$ & $\mathrm{n} 59125 \mathrm{f}$ & 1993 Jul 15 & $04: 27$ & 9183.69 & A & $5.0 \times 7.5$ & 90 & 3.2 & 9 & $4540-5700$ & n59183a \\
\hline 1993 May 19 & $04: 36$ & 9126.69 & F & $3.2 \times 6.4$ & 90 & & 5 & $4720-7160$ & n59126f & $1993 \mathrm{Jul} 19$ & 03:48 & 9187.66 & $\mathrm{~F}$ & $3.2 \times 6.4$ & 90 & & 5 & $4660-7170$ & n59187f \\
\hline 1993 May 20 & 04:16 & 9127.68 & $\mathrm{~F}$ & $3.2 \times 6.4$ & 90 & & 5 & $4610-7170$ & n59127f & $1993 \mathrm{Jul} 20$ & 04:06 & 9188.67 & $\mathrm{~F}$ & $3.2 \times 6.4$ & 90 & & 5 & $4660-7170$ & n59188f \\
\hline 1993 May 21 & $05: 55$ & 9128.75 & A & $5.0 \times 7.5$ & 90 & 3.7 & 9 & $4530-5670$ & n59128a & $1993 \mathrm{Jul} 21$ & 04:31 & 9189.69 & F & $3.2 \times 6.4$ & 90 & & 5 & $4610-7220$ & n59189f \\
\hline 1993 May 21 & $05: 28$ & 9128.73 & $\mathrm{~F}$ & $3.2 \times 6.4$ & 90 & & 5 & $4660-7170$ & n59128f & 1993 Jul 22 & $03: 42$ & 9190.65 & A & $5.0 \times 7.5$ & 90 & 3.1 & 9 & $4560-5720$ & n59190a \\
\hline 1993 May 21 & $23: 05$ & 9129.46 & W & $3.0 \times 10.0$ & 90 & 3 & 9 & $4430-5620$ & $\mathrm{n} 59129 \mathrm{w}$ & $1993 \mathrm{Jul} 23$ & 03:53 & 9191.66 & F & $3.2 \times 6.4$ & 90 & & 5 & $4660-7170$ & n59191f \\
\hline 1993 May 22 & $22: 55$ & 9130.46 & W & $3.0 \times 10.0$ & 90 & 2 & 9 & $4410-5560$ & n59130wa & $1993 \mathrm{Jul} 28$ & 06:05 & 9196.77 & $\mathrm{H}$ & $4.0 \times 10.0$ & 60 & $1-1.5$ & 8 & $3140-8030$ & $\mathrm{n} 59196 \mathrm{~h}$ \\
\hline 1993 May 23 & $00: 42$ & 9130.53 & W & $3.0 \times 10.0$ & 90 & 2.5 & 9 & $6100-7230$ & n59130wb & 1993 Jul 29 & $04: 52$ & 9197.70 & A & $5.0 \times 7.5$ & 90 & 3.6 & 9 & $4530-5690$ & n59197a \\
\hline 1993 May 23 & $20: 17$ & 9131.35 & B & $15.0 \times 11.0$ & 3.6 & $2-3$ & 15 & $4510-7910$ & n59131b & 1993 Aug 6 & $04: 23$ & 9205.68 & A & $5.0 \times 7.5$ & 90 & 3.5 & 9 & $4540-5700$ & n59205a \\
\hline 1993 May 23 & $20: 30$ & 9131.35 & $\mathrm{~W}$ & $3.0 \times 10.0$ & 90 & 1 & 9 & $4470-5630$ & n59131wa & 1993 Aug 12 & 03:52 & 9211.66 & A & $5.0 \times 7.5$ & 90 & 3.5 & 9 & $4520-5680$ & n59211a \\
\hline 1993 May 23 & $22: 50$ & 9131.45 & W & $3.0 \times 10.0$ & 90 & 1 & 9 & $6060-7190$ & n59131wb & 1993 Aug 13 & $05: 44$ & 9212.74 & $\mathrm{H}$ & $4.0 \times 10.0$ & 60 & 1.5 & 8 & $3120-8020$ & $\mathrm{n} 59212 \mathrm{~h}$ \\
\hline 1993 May 24 & 13:09 & 9132.05 & E & $5.0 \times 8.4$ & 90 & & 5 & $4670-5330$ & n59132e & 1993 Sep 10 & 03:08 & 9240.63 & A & $5.0 \times 7.5$ & 90 & 4.4 & 9 & $4530-5690$ & n59240a \\
\hline 1993 May 24 & $21: 35$ & 9132.40 & W & $3.0 \times 10.0$ & 90 & 2 & 9 & $4430-5600$ & n59132wa & 1993 Sep 10 & 04:02 & 9240.67 & $\mathrm{H}$ & $4.0 \times 10.0$ & 62 & 1 & 8 & $3120-9900$ & $\mathrm{n} 59240 \mathrm{~h}$ \\
\hline 1993 May 24 & $23: 45$ & 9132.49 & $\mathrm{~W}$ & $3.0 \times 10.0$ & 90 & 2 & 9 & $6110-7230$ & n59132wb & 1993 Sep 12 & 04:05 & 9242.67 & $\mathrm{H}$ & $4.0 \times 10.0$ & 59 & 2 & 8 & $3160-8040$ & $\mathrm{n} 59242 \mathrm{~h}$ \\
\hline 1993 May 28 & $06: 19$ & 9135.76 & A & $5.0 \times 7.5$ & 90 & 3.1 & 9 & $4510-5660$ & n59135a & 1993 Sep 13 & 03:54 & 9243.66 & $\mathrm{H}$ & $4.0 \times 10.0$ & 61 & 2 & 8 & $3120-8040$ & $\mathrm{n} 59243 \mathrm{~h}$ \\
\hline 1993 May 28 & 09:53 & 9135.92 & $\mathrm{~F}$ & $3.2 \times 6.4$ & 90 & & 5 & $4660-7140$ & n59135f & 1993 Sep 25 & $03: 15$ & 9255.63 & $\mathrm{H}$ & $4.0 \times 10.0$ & 59 & 1.5 & 8 & $3200-8030$ & $\mathrm{n} 59255 \mathrm{~h}$ \\
\hline 1993 Jun 1 & $23: 00$ & 9140.46 & W & $3.0 \times 10.0$ & 90 & 3 & 9 & $4380-5570$ & $\mathrm{n} 59140 \mathrm{w}$ & & & & & & & & & & \\
\hline 1993 Jun 2 & $21: 25$ & 9141.39 & W & $3.0 \times 10.0$ & 90 & 3 & 9 & $4360-5550$ & n59141wa & & & & & & & & & & \\
\hline 1993 Jun 2 & $23: 10$ & 9141.46 & W & $3.0 \times 10.0$ & 90 & 3 & 9 & $6230-7350$ & n59141wb & & & & & & & & & & \\
\hline 1993 Jun 4 & $05: 20$ & 9142.72 & A & $5.0 \times 7.5$ & 90 & 4.5 & 9 & $4500-5650$ & n59142a & & & & & & & & & & \\
\hline 1993 Jun 11 & $05: 47$ & 9149.74 & A & $5.0 \times 7.5$ & 90 & 4.2 & 9 & $4500-5660$ & n59149a & & & & & & & & & & \\
\hline 1993 Jun 12 & $08: 22$ & 9150.85 & $\mathrm{~F}$ & $3.2 \times 6.4$ & 90 & & 5 & $4720-7170$ & n59150f & & & & & & & & & & \\
\hline 1993 Jun 13 & $03: 52$ & 9151.66 & $\mathrm{~F}$ & $3.2 \times 6.4$ & 90 & & 5 & $4720-7170$ & n59151f & & & & & & & & & & \\
\hline 1993 Jun 14 & 04:01 & 9152.67 & $\mathrm{~F}$ & $3.2 \times 6.4$ & 90 & & 5 & $4720-7170$ & $\mathrm{n} 59152 \mathrm{f}$ & & & & & & & & & & \\
\hline 1993 Jun 16 & $20: 25$ & 9155.41 & W & $3.0 \times 10.0$ & 90 & $2-5$ & 9 & $4310-5450$ & n59155wa & & & & & & & & & & \\
\hline 1993 Jun 16 & $23: 05$ & 9155.46 & W & $3.0 \times 10.0$ & 90 & 2 & 9 & $6090-7220$ & $\mathrm{n} 59155 \mathrm{wb}$ & & & & & & & & & & \\
\hline 1993 Jun 17 & 04:01 & 9155.67 & $\mathrm{~F}$ & $3.2 \times 6.4$ & 90 & & 5 & $4720-7170$ & n59155f & & & & & & & & & & \\
\hline 1993 Jun 17 & $21: 05$ & 9156.39 & W & $3.0 \times 10.0$ & 90 & 3 & 9 & $4310-5510$ & n59156wa & & & & & & & & & & \\
\hline 1993 Jun 17 & $23: 05$ & 9156.46 & W & $3.0 \times 10.0$ & 90 & 3 & 9 & $6020-7160$ & $\mathrm{n} 59156 \mathrm{wb}$ & & & & & & & & & & \\
\hline 1993 Jun 18 & 04:00 & 9156.67 & $\mathrm{~F}$ & $3.2 \times 6.4$ & 90 & & 5 & $4720-7170$ & n59156f & & & & & & & & & & \\
\hline 1993 Jun 18 & $05: 59$ & 9156.75 & A & $5.0 \times 7.5$ & 90 & 4.2 & 9 & $4530-5690$ & n59156a & & & & & & & & & & \\
\hline 1993 Jun 18 & $21: 45$ & 9157.41 & W & $3.0 \times 10.0$ & 90 & 2 & 9 & $4240-5450$ & n59157wa & & & & & & & & & & \\
\hline 1993 Jun 18 & $22: 50$ & 9157.45 & W & $3.0 \times 10.0$ & 90 & 2 & 9 & $6080-7210$ & n59157wb & & & & & & & & & & \\
\hline 1993 Jun 19 & 04:01 & 9157.67 & $\mathrm{~F}$ & $3.2 \times 6.4$ & 90 & & 5 & $4660-7170$ & n59157f & & & & & & & & & & \\
\hline 1993 Jun 19 & $20: 20$ & 9158.35 & $\mathrm{~W}$ & $3.0 \times 10.0$ & 90 & 2 & 9 & $4350-5530$ & n59158wa & & & & & & & & & & \\
\hline 1993 Jun 19 & $21: 20$ & 9158.39 & W & $3.0 \times 10.0$ & 90 & 2 & 9 & $6080-7200$ & n59158wb & & & & & & & & & & \\
\hline 1993 Jun 20 & $03: 55$ & 9158.66 & $\mathrm{~F}$ & $3.2 \times 6.4$ & 90 & & 5 & $4660-7170$ & n59158f & & & & & & & & & & \\
\hline 1993 Jun 20 & $20: 15$ & 9159.34 & $\mathrm{~W}$ & $3.0 \times 10.0$ & 90 & 1.5 & 9 & $6080-7230$ & n59159wb & & & & & & & & & & \\
\hline 1993 Jun 20 & $21: 15$ & 9159.38 & $\mathrm{~W}$ & $3.0 \times 10.0$ & 90 & 1.5 & 9 & $4340-5550$ & n59159wa & & & & & & & & & & \\
\hline
\end{tabular}

NOTE.-Codes for data origin in col. (4): (A) $1.8 \mathrm{~m}$ Perkins telescope + Ohio State CCD spectrograph; (B) $1.0 \mathrm{~m}$ Wise telescope + CCD spectrograph; (E) $1.8 \mathrm{~m}$ DAO telescope + CCD spectrograph; (F) $1.6 \mathrm{~m}$ Mount Hopkins telescope + Reticon scanner; $(\mathrm{H}) 3.0 \mathrm{~m}$ Shane telescope + Kast spectrograph; $(\mathrm{J}) 2.1 \mathrm{~m}$ McDonald telescope + Cassegrain Grating spectrograph; (L) $6.0 \mathrm{~m}$ Special Astrophysical Observatory + TV scanner; (M) $2.2 \mathrm{~m}$ Calar Alto Observatory + CCD spectrographs; (R) $1.5 \mathrm{~m}$ Loiano telescope + CCD spectrograph; (T) $4.2 \mathrm{~m}$ William Herschel Telescope + CCD spectrograph; (U) $1.9 \mathrm{~m} \mathrm{SAAO}$ telescope + Reticon; (W) $2.6 \mathrm{~m}$ Shajn telescope + CCD spectrograph; (Y) $2.2 \mathrm{~m}$ Beijing Astronomical Observatory telescope + CCD spectrograph; (Z) $2.2 \mathrm{~m}$ ESO telescope + CCD spectrograph. 
$V=0.71, V-R=0.39$, and $R-I=0.31 \mathrm{mag}$. As described by Romanishin et al., it is also possible to calibrate high-quality CCD images by measuring the brightness of the host galaxy in carefully chosen annuli. When possible, we used such measurements as a check on the absolute calibration. Formal errors, based on counting statistics, are in every case $\sim 0.01$ mag unless otherwise noted.

CCD images were obtained in BVRI with the Lowell Observatory $1.1 \mathrm{~m}$ telescope, as given in Table 4 . The filters and response function are as described by Beckert \& Newberry (1989). Columns (1) and (2) give the UT date and time of observation, and the corresponding Julian Date is given in column (3). The magnitudes, as measured through the bandpass and aperture radius given in columns (4) and (5), respectively, are given in column (6).

In Table 5 we give $V$-band measurements made with a CCD camera on the Michigan State University $0.6 \mathrm{~m}$ telescope in East Lansing, Michigan. This system is described by Smith et al. (1994). Table 6 gives the measurements from images obtained with a CCD camera on the $2.0 \mathrm{~m}$ telescope at San Pedro Mártir, Baja California. The detector is a Thompson THX31 156 chip with a Metachrome II UV coating. The pixel scale is 0.26 pixel $^{-1}$. Measurements made with the OPTEC CCD system on the Behlen Observatory $0.76 \mathrm{~m}$ telescope near Mead, Nebraska, are given in Table 7.

A program of photoelectric aperture photometry was carried out with the $0.60 \mathrm{~m}$ telescope of the Crimean Laboratory

TABLE 4

LOWELL $1.1 \mathrm{~m}$ CCD PHOTOMETRY

\begin{tabular}{cccccc}
\hline UT & & Julian Date & & Aperture & \\
Date & Time & $(2440000+)$ & Filter & $($ arcsec $)$ & magnitude \\
(1) & $(2)$ & $(3)$ & $(4)$ & $(5)$ & $(6)$ \\
\hline 1993 Mar 17 & $07: 27$ & 9063.81 & $B$ & 8.0 & 14.16 \\
1993 Mar 17 & $07: 40$ & 9063.82 & $V$ & 8.0 & 13.49 \\
1993 Mar 17 & $07: 52$ & 9063.83 & $R$ & 8.0 & 12.88 \\
1993 Mar 17 & $08: 03$ & 9063.84 & $I$ & 8.0 & 12.50 \\
1993 Apr 1 & $09: 26$ & 9078.89 & $B$ & 8.0 & 14.26 \\
1993 Apr 1 & $09: 37$ & 9078.90 & $V$ & 8.0 & 13.57 \\
1993 Apr 1 & $09: 45$ & 9078.91 & $R$ & 8.0 & 12.94 \\
1993 Apr 1 & $09: 56$ & 9078.91 & $I$ & 8.0 & 12.54 \\
1993 Aug 14 & $02: 54$ & 9213.62 & $V$ & 8.0 & 13.52 \\
1993 Aug 14 & $02: 54$ & 9213.62 & $V$ & 25.0 & 12.93 \\
1993 Aug 14 & $03: 16$ & 9213.64 & $R$ & 8.0 & 12.93 \\
1993 Aug 14 & $03: 16$ & 9213.64 & $R$ & 25.0 & 12.36 \\
1993 Aug 14 & $03: 37$ & 9213.65 & $I$ & 8.0 & 12.53 \\
1993 Aug 14 & $03: 37$ & 9213.65 & $I$ & 25.0 & 11.91 \\
1993 Aug 14 & $04: 03$ & 9213.67 & $B$ & 8.0 & 14.18 \\
1993 Aug 14 & $04: 03$ & 9213.67 & $B$ & 25.0 & 13.63 \\
1993 Aug 15 & $02: 47$ & 9214.62 & $I$ & 8.0 & 12.52 \\
1993 Aug 15 & $02: 47$ & 9214.62 & $I$ & 25.0 & 11.89 \\
1993 Aug 15 & $03: 14$ & 9214.63 & $B$ & 8.0 & 14.17 \\
1993 Aug 15 & $03: 14$ & 9214.63 & $B$ & 25.0 & 13.63 \\
1993 Aug 15 & $03: 39$ & 9214.65 & $V$ & 8.0 & 13.51 \\
1993 Aug 15 & $03: 39$ & 9214.65 & $V$ & 25.0 & 12.93 \\
1993 Aug 15 & $04: 00$ & 9214.67 & $R$ & 8.0 & 12.92 \\
1993 Aug 15 & $04: 00$ & 9214.67 & $R$ & 25.0 & 12.37 \\
1993 Aug 16 & $02: 43$ & 9215.61 & $I$ & 8.0 & 12.53 \\
1993 Aug 16 & $02: 43$ & 9215.61 & $I$ & 25.0 & 11.88 \\
1993 Aug 16 & $03: 04$ & 9215.63 & $R$ & 8.0 & 12.92 \\
1993 Aug 16 & $03: 04$ & 9215.63 & $R$ & 25.0 & 12.37 \\
1993 Aug 16 & $03: 30$ & 9215.65 & $B$ & 8.0 & 14.16 \\
1993 Aug 16 & $03: 30$ & 9215.65 & $B$ & 25.0 & 13.64 \\
1993 Aug 16 & $03: 56$ & 9215.66 & $V$ & 8.0 & 13.50 \\
1993 Aug 16 & $03: 56$ & 9215.66 & $V$ & 25.0 & 12.93 \\
\hline
\end{tabular}

TABLE 5

MSU $0.5 \mathrm{~m} V$-BAND CCD PHOTOMETRY

\begin{tabular}{ccccc}
\hline \hline UT & & Julian Date & \multicolumn{2}{c}{$V$ (magnitudes) } \\
Date & Time & $(2440000+)$ & $\left(R=7^{\prime \prime}\right)$ & $\left(R=9^{\prime \prime} .8\right)$ \\
$(1)$ & $(2)$ & $(3)$ & $(4)$ & $(5)$ \\
\hline 1993 Apr 18 & $04: 32$ & 9095.69 & 13.62 & 13.43 \\
1993 Apr 22 & $04: 38$ & 9099.69 & 13.59 & 13.39 \\
1993 Apr 22 & $04: 42$ & 9099.70 & 13.59 & 13.37 \\
1993 Apr 23 & $04: 42$ & 9100.70 & 13.59 & 13.39 \\
1993 Apr 27 & $06: 03$ & 9104.75 & 13.54 & 13.36 \\
1993 May 14 & $04: 41$ & 9121.69 & 13.49 & 13.31 \\
1993 May 17 & $04: 31$ & 9124.69 & 13.49 & 13.33 \\
1993 May 26 & $03: 54$ & 9133.66 & 13.54 & 13.35 \\
1993 Jun 16 & $04: 34$ & 9154.69 & 13.62 & 13.40 \\
\hline
\end{tabular}

Sternberg Institute (CLSI) at Nauchny, and the $V$-band measurements are given in Table 8. Photoelectric aperture photometry was also undertaken with the $1.25 \mathrm{~m}$ telescope of the Crimean Astrophysical Observatory (CAO), and the $V$-band measurements obtained are given in Table 9. Here the calibration is based on stars from the list of Lyutyi (1972).

$V$-band measurements obtained with an RCA CCD on the Center for Basement Astrophysics (CBA) $0.3 \mathrm{~m}$ telescope in Laurel, Maryland, are given in Table 10. This system is described by Skillman \& Patterson (1993).

\section{ANALYSIS OF THE DATA}

\subsection{Intercalibration of the HST FOS and the IUE SWP Spectra}

In order that we may combine the light curves from the IUE and HST campaigns, the two sets of data should be on as similar flux scales as possible. Because the NEWSIPS calibration of the IUE SWP data is based upon a white dwarf model atmosphere of G191-B2B, the major step involved converting the FOS flux calibration scale to the same basis. The conversion function between the two calibration bases is known (Bohlin 1993a, b) and was simply multiplied into the FOS data. The uncertainty in this new absolute scale is still under investigation but is thought to lie between $2 \%$ and $4 \%$, the larger value applicable to the sub-Ly $\alpha$ region of the spectra presented here (Bohlin 1994). In the top panel of Figure 5 we show a direct comparison between the mean SWP and corrected FOS spectra smoothed to SWP spectral resolution in the 20 cases where there were contemporaneous observations in both instruments. In the bottom panel of Figure 5 we show the ratio. The SWP spectra were first shifted onto the peak of the CrV emis-

TABLE 6

SAN PEDRo MÁrTIR $2.0 \mathrm{~m} V$-BAND CCD PHOTOMETRY

\begin{tabular}{ccccc}
\hline \hline UT & Julian Date & \multicolumn{2}{c}{$V$ (magnitudes) } \\
Date & Time & $\begin{array}{c}\text { (2440000+) } \\
\left(R=8^{\prime \prime}\right)\end{array}$ & $\begin{array}{c}\left(R=25^{\prime \prime}\right) \\
(4)\end{array}$ & $(5)$ \\
\hline 1993 Apr 20 & $09: 38$ & 9097.90 & 13.62 & 12.97 \\
1993 Apr 21 & $11: 25$ & 9098.98 & 13.60 & 12.96 \\
1993 Apr 22 & $07: 22$ & 9099.81 & 13.60 & 12.96 \\
1993 Apr 24 & $09: 27$ & 9101.89 & 13.59 & 12.96 \\
\hline
\end{tabular}


TABLE 7

BEHLEN OBSERVATORY $0.76 \mathrm{~m} V$-BAND CCD PHOTOMETRY

\begin{tabular}{cccc}
\hline \hline UT & Julian Date & $V$ (magnitudes) \\
$\begin{array}{c}\text { Date } \\
(1)\end{array}$ & $\begin{array}{c}\text { Time } \\
(2)\end{array}$ & $\begin{array}{c}\text { (2440000+) } \\
(3)\end{array}$ & $(4)$ \\
\hline 1993 Mar 27 & $10: 46$ & 9073.95 & $13.50 \pm 0.02$ \\
1993 Apr 21 & $06: 07$ & 9098.75 & $13.55 \pm 0.04$ \\
1993 Apr 24 & $06: 10$ & 9101.76 & $13.48 \pm 0.02$ \\
1993 May 13 & $08: 44$ & 9120.86 & $13.44 \pm 0.04$ \\
1993 May 14 & $07: 04$ & 9121.79 & $13.38 \pm 0.02$ \\
1993 May 20 & $08: 26$ & 9127.85 & $13.41 \pm 0.03$ \\
\hline
\end{tabular}

sion line, whose wavelength was set by the mean FOS spectrum. A similar ratio with some dispersion is observed in the individual spectra as well. The ratio varies at about the $\pm 6 \%$ level, with some systematic trend that the SWP/FOS ratio is greater than 1 at the longer wavelengths and less than 1 at the shorter wavelengths. We note that SWP 47505 was the only SWP spectrum which had a lower flux at every wavelength than its contemporaneous FOS spectrum (JD 2,449,097).

Looking at the ratio in Figure 5 in more detail, the systematically lower SWP flux at the shorter wavelengths is almost certainly due to the SWP nonlinearity at low count rates. The NEWSIPS calibration removed part of this effect, but some residual nonlinearity is certainly still present, especially in the lower state spectra. Some of the structure in the ratio is due to artifacts in the SWP spectra (e.g., between roughly 1470 and $1500 \AA$ ). The "flip-flop" effect in the region near the position of the C IV emission line is not simply a problem of zero-point wavelength misalignment, although the uncertainty in the NEWSIPS SWP wavelength scale could in turn produce an error in the flux calibration in this region. The higher SWP flux at longer wavelengths is not understood. The offset is apparently multiplicative, since it is the same for the $\mathrm{C}$ III broad emission feature as it is in the neighboring continuum (which in principle could have an extended nonnuclear contribution in the

TABLE 8

CRIMEAN LABORATORY STERNBERG INSTITUTE $0.6 \mathrm{~m} V$-BAND PHOTOELECTRIC PHOTOMETRY

\begin{tabular}{cccc}
\hline UT & & Julian Date & $V$ (magnitudes) \\
Date & Time & $(2440000+)$ & $\left(R=7^{\prime \prime} .15\right)$ \\
$(1)$ & $(2)$ & $(3)$ & $(4)$ \\
\hline 1993 Jan 4 & $02: 49$ & 8991.62 & $13.47 \pm 0.01$ \\
1993 Mar 1 & $02: 19$ & 9047.60 & $13.59 \pm 0.02$ \\
1993 Mar 12 & $21: 32$ & 9059.40 & $13.56 \pm 0.02$ \\
1993 Mar 15 & $01: 47$ & 9061.57 & $13.56 \pm 0.02$ \\
1993 Mar 16 & $01: 24$ & 9062.56 & $13.57 \pm 0.02$ \\
1993 Mar 17 & $00: 52$ & 9063.54 & $13.59 \pm 0.01$ \\
1993 Mar 22 & $22: 42$ & 9069.45 & $13.61 \pm 0.02$ \\
1993 Apr 13 & $20: 46$ & 9091.37 & $13.68 \pm 0.03$ \\
1993 Apr 21 & $22: 19$ & 9099.43 & $13.67 \pm 0.01$ \\
1993 Apr 22 & $21: 39$ & 9100.40 & $13.63 \pm 0.02$ \\
1993 Apr 24 & $21: 12$ & 9102.38 & $13.64 \pm 0.02$ \\
1993 May 23 & $21: 10$ & 9131.38 & $13.55 \pm 0.02$ \\
1993 Jun 7 & $19: 14$ & 9146.30 & $13.59 \pm 0.01$ \\
1993 Jun 9 & $19: 34$ & 9148.32 & $13.62 \pm 0.02$ \\
1993 Jun 10 & $21: 00$ & 9149.37 & $13.65 \pm 0.02$ \\
1993 Jun 11 & $20: 40$ & 9150.36 & $13.63 \pm 0.02$ \\
\hline
\end{tabular}

TABLE 9

CRIMEAN ASTROPHYSICAL OBSERVATORY $1.25 \mathrm{~m} V$-BAND PHOTOELECTRIC PHOTOMETRY

\begin{tabular}{|c|c|c|c|}
\hline \multicolumn{2}{|l|}{ UT } & \multirow{2}{*}{$\begin{array}{c}\text { Julian Date } \\
(2440000+) \\
(3)\end{array}$} & \multirow{2}{*}{$\begin{array}{c}V \text { (magnitudes) } \\
\left(R=7^{\prime \prime} .5\right) \\
(4)\end{array}$} \\
\hline $\begin{array}{c}\text { Date } \\
(1)\end{array}$ & $\begin{array}{c}\text { Time } \\
(2)\end{array}$ & & \\
\hline 1993 Apr 26 & $20: 57$ & 9104.37 & $13.56 \pm 0.01$ \\
\hline 1993 Apr 27 & $20: 29$ & 9105.35 & $13.51 \pm 0.01$ \\
\hline 1993 May 13 & $21: 22$ & 9121.39 & $13.50 \pm 0.03$ \\
\hline 1993 Jun 10 & $19: 51$ & 9149.33 & $13.57 \pm 0.01$ \\
\hline 1993 Jun 20 & $20: 55$ & 9159.37 & $13.58 \pm 0.02$ \\
\hline 1993 Jun 23 & $20: 22$ & 9162.35 & $13.57 \pm 0.01$ \\
\hline
\end{tabular}

larger aperture SWP spectra). Whatever the origins of the differences, they are acceptably small. We compare spectral measurements for contemporaneous FOS SWP spectra in $\S 5.3$ below.

Finally, we note that, based upon the presence of the strong narrow He II $\lambda 1640$ feature observed peaking near $1667 \AA$ in the FOS spectra, a significant fraction of the same feature observed in the SWP spectra must also be narrow He II emission, and not just the SWP reseau artifact nominally located at $1663 \AA$.

\subsection{The FOS Spectral Measurements}

In this paper all ultraviolet measurements were made in the observed frame and then converted to and presented in the rest frame. Wavelengths referring to spectral regions, such as measurement windows, will be quoted in the observed frame for easy referencing with the spectra. The component fluxes and wavelengths designating the name of an emission line or continuum band will be quoted in the rest frame, for $z=$ 0.0174 . The continuum fluxes $\left(F_{\lambda}\right)$ and emission-line fluxes will be higher than those measured in the observed frame by factors of $(1+z)^{3}$ and $(1+z)^{2}$, respectively. Two methods of emission-line and continuum measurements were employed here: direct integration and spectral fitting. We describe these below.

\subsubsection{Direct Integration}

Eight "continuum" windows, generally $20 \AA$ wide in the observed frame, were chosen by inspection of the mean FOS spectrum. We emphasize that none of the so-called continuum windows measure a true continuum level; broad emission line wings, the Balmer recombination continuum, and the Fe II

TABLE 10

CBA $0.3 \mathrm{~m} V$-BAND CCD PHOTOMETRY

\begin{tabular}{cccc}
\hline \hline UT & & Julian Date & $V$ (magnitudes) \\
Date & Time & $(2440000+)$ & $\left(R=8^{\prime \prime}\right)$ \\
$(1)$ & $(2)$ & $(3)$ & $(4)$ \\
\hline 1993 Mar 7 & $06: 45$ & 9053.78 & $13.62 \pm 0.06$ \\
1993 Apr 24 & $05: 25$ & 9101.73 & $13.68 \pm 0.06$ \\
1993 Apr 30 & $01: 14$ & 9107.55 & $13.68 \pm 0.06$ \\
1993 May 7 & $02: 06$ & 9114.59 & $13.67 \pm 0.06$ \\
\hline
\end{tabular}



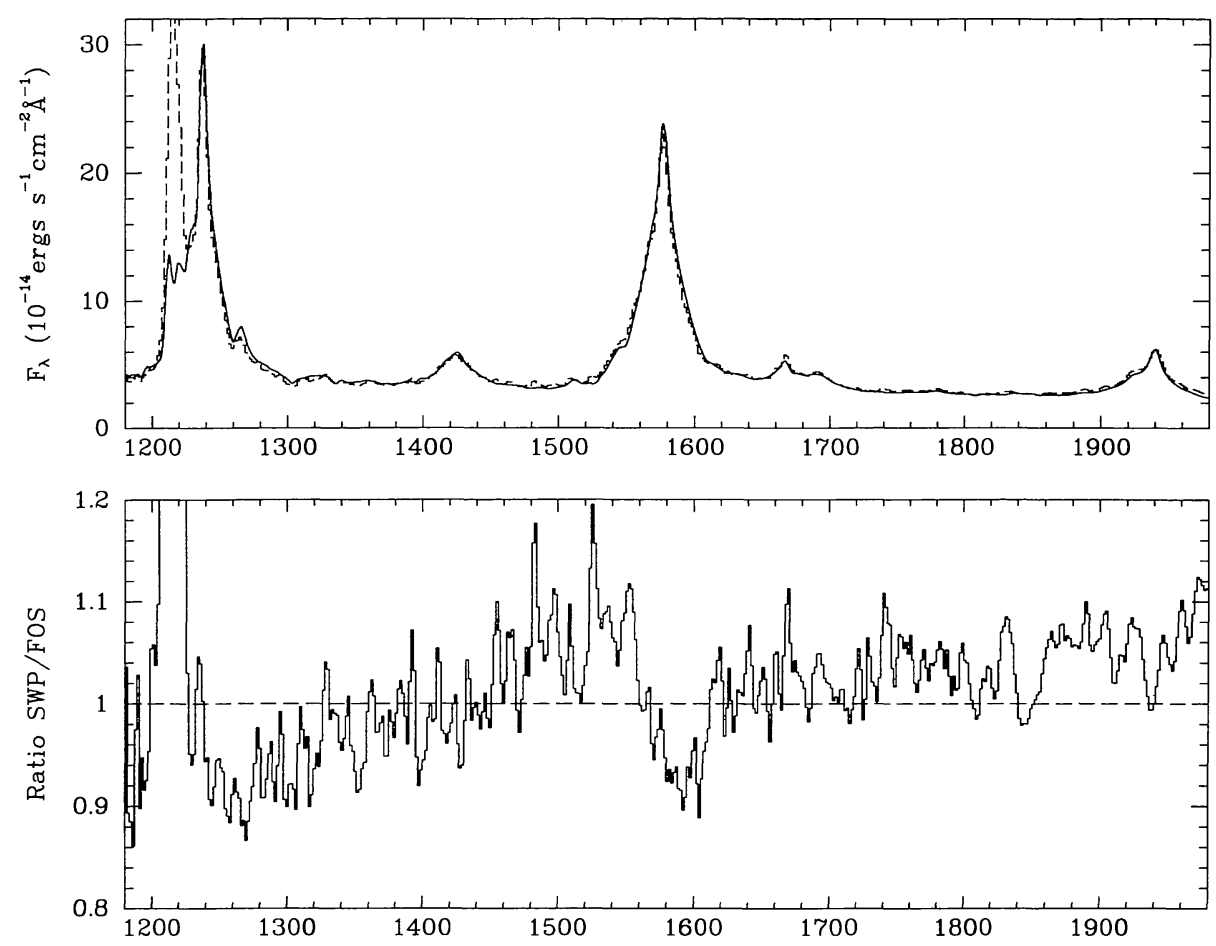

FIG. 5.-Top panel: Comparison of the mean FOS spectrum (solid line), smoothed to the SWP resolution, with the mean SWP spectrum (dashed line). Only contemporaneous FOS SWP observations were included in the means. Bottom panel: Ratio of the mean SWP spectrum to the mean FOS spectrum. The abscissa has units of observed wavelength $(\AA)$.

pseudo-continuum contaminate these windows to various extents. These continuum windows will be designated by the mean rest wavelength within each window. Table 11 lists and defines these continuum windows. A mean flux per unit wavelength was determined in each continuum window. The fluxes in six of the continuum windows are tabulated in the rest frame with their statistical errors in Table 12 , in units of $10^{-14}$ ergs $\mathrm{s}^{-1} \mathrm{~cm}^{-2} \AA^{-1}$. The statistical errors in this and all subsequent ultraviolet light-curve tables are given to the same number of significant figures as the flux value. The Julian Date in this and all subsequent FOS light-curve tables refers to the mean starting time for the two separate $\mathrm{G} 130 \mathrm{H}$ and $\mathrm{G} 190 \mathrm{H}$ exposures. We plot these continuum light curves in Figure 6. We stress that we place most confidence in light-curve "events" of durations 3 days or more. Single-day "events," such as the dimples which occur near JD 2,449,101 and JD 2,449,131, are considered suspect because of the systematic photometric errors encountered in the FOS spectra (see $\S 2$ and Appendix D). The $2195 \AA$ band suffers the most from these errors.

The continuum level underneath an emission line was determined by a linear interpolation between the nearest two continuum windows on either side of the emission line. The emission-line flux was then integrated within its designated wavelength interval after removing this interpolated continuum. Table 11 lists the wavelength intervals over which the various emission features were integrated. We followed the lead of past attempts of measuring the kinematics of the C IV $\lambda 1549$ emission-line region (Clavel 1991) and defined red and blue cores corresponding to emission-line flux falling within $3000 \mathrm{~km} \mathrm{~s}^{-1}$ redward and blueward, respectively, of the emission-line peak at approximately $1576 \AA$ in the observed frame. The blue wing was then arbitrarily defined to extend to $1519 \AA$, near the local minimum just redward of the $N$ IV ] $\lambda 1486$ emission line. We defined the red wing to extend to a velocity symmetric about the peak with the blue wing, near

TABLE 11

FOS DIRECT-INTEGRATION WAVELENGTH WINDOWS

\begin{tabular}{|c|c|}
\hline Component $^{a}$ & Wavelength Window ${ }^{b}$ \\
\hline$F_{\lambda}(1145 \AA)$ & $1155-1175 \AA$ \\
\hline$F_{\lambda}(1350 \AA)$ & $1370-1380 \AA$ \\
\hline$F_{\lambda}(1460 \AA)$ & $1475-1495 \AA$ \\
\hline$F_{\lambda}(1720 \AA)$ & $1740-1760 \AA$ \\
\hline$F_{\lambda}(1790 \AA)$ & $1810-1830 \AA$ \\
\hline$F_{\lambda}(1985 \AA)$ & $2010-2030 \AA$ \\
\hline$F_{\lambda}(2030 \AA)$ & $2056-2076 \AA$ \\
\hline$F_{\lambda}(2195 \AA)$ & $2224-2242 \AA$ \\
\hline Ly $\alpha \lambda 1216$ (core) & $1223-1250 \AA$ \\
\hline $\mathrm{NV} \lambda 1240$ & $1260-1300 \AA$ \\
\hline Si IV $\lambda 1400+$ O IV] $\lambda 1402$ & $1380-1455 \AA$ \\
\hline $\mathrm{C}$ гV $\lambda 1549$ & $1519-1633 \AA$ \\
\hline Crv Blue Wing & $1519-1560 \AA$ \\
\hline Crv Blue Core & $1560-1576 \AA$ \\
\hline Civ Red Core & $1576-1592 \AA$ \\
\hline Crv Red Wing & $1592-1633 \AA$ \\
\hline He II $\lambda 1640+$ OIII] $\lambda 1663$ & $1645-1730 \AA$ \\
\hline $\mathrm{C}_{\text {III] }} \lambda 1909+\mathrm{Si}$ III] $\lambda 1893$ & $1865-2010 \AA$ \\
\hline
\end{tabular}

${ }^{a}$ Component name uses rest-frame wavelengths.

${ }^{\mathrm{b}}$ Window given in observed frame. 
TABLE 12

FOS CONTINUUM BANDS ${ }^{\mathrm{a}}$

\begin{tabular}{|c|c|c|c|c|c|c|}
\hline $\begin{array}{l}\text { Julian Date } \\
(2440000+)\end{array}$ & $F_{\lambda}(1145 \AA)$ & $F_{\lambda}(1350 \AA)$ & $F_{\lambda}(1460 \AA)$ & $F_{\lambda}(1790 \AA)$ & $F_{\lambda}(2030 \AA)$ & $F_{\lambda}(2195 \AA)$ \\
\hline 9097.00 & $2.56 \pm 0.123$ & $2.48 \pm 0.0468$ & $2.25 \pm 0.0262$ & $1.95 \pm 0.0183$ & $1.61 \pm 0.0119$ & $1.56 . \pm 0.0101$ \\
\hline 9098.07 & $2.76 \pm 0.126$ & $2.68 \pm 0.0486$ & $2.42 \pm 0.0271$ & $2.09 \pm 0.0189$ & $1.70 \pm 0.0122$ & $1.63 \pm 0.0103$ \\
\hline 9099.01 & $2.75 \pm 0.240$ & $2.77 \pm 0.0769$ & $2.52 \pm 0.0405$ & $2.23 \pm 0.0195$ & $1.84 \pm 0.0127$ & $1.72 \pm 0.0106$ \\
\hline 9100.01 & $3.00 \pm 0.132$ & $3.01 \pm 0.0514$ & $2.68 \pm 0.0285$ & $2.27 \pm 0.0197$ & $1.81 \pm 0.0126$ & $1.66 \pm 0.0104$ \\
\hline 9101.02 & $3.09 \pm 0.132$ & $2.88 \pm 0.0502$ & $2.60 \pm 0.0281$ & $2.22 \pm 0.0195$ & $1.75 \pm 0.0124$ & $1.62 \pm 0.0103$ \\
\hline 9102.02 & $3.04 \pm 0.133$ & $3.35 \pm 0.0542$ & $3.00 \pm 0.0301$ & $2.49 \pm 0.0206$ & $1.94 \pm 0.0130$ & $1.80 \pm 0.0108$ \\
\hline 9103.09 & $3.87 \pm 0.283$ & $3.43 \pm 0.0964$ & $3.11 \pm 0.0509$ & $2.63 \pm 0.0212$ & $2.08 \pm 0.0135$ & $1.93 \pm 0.0112$ \\
\hline 9104.03 & $3.88 \pm 0.145$ & $3.60 \pm 0.0561$ & $3.24 \pm 0.0313$ & $2.67 \pm 0.0214$ & $2.19 \pm 0.0138$ & $2.04 \pm 0.0115$ \\
\hline 9105.03 & $3.83 \pm 0.145$ & $3.70 \pm 0.0568$ & $3.38 \pm 0.0320$ & $2.81 \pm 0.0219$ & $2.21 \pm 0.0139$ & $2.05 \pm 0.0115$ \\
\hline 9106.03 & $4.23 \pm 0.150$ & $3.74 \pm 0.0571$ & $3.29 \pm 0.0315$ & $2.80 \pm 0.0219$ & $2.20 \pm 0.0139$ & $1.94 \pm 0.0112$ \\
\hline 9106.97 & $3.62 \pm 0.144$ & $3.45 \pm 0.0550$ & $3.13 \pm 0.0308$ & $2.63 \pm 0.0212$ & $2.12 \pm 0.0136$ & $1.94 \pm 0.0112$ \\
\hline 9107.98 & $4.17 \pm 0.152$ & $3.51 \pm 0.0555$ & $3.23 \pm 0.0313$ & $2.72 \pm 0.0216$ & $2.19 \pm 0.0139$ & $1.99 \pm 0.0114$ \\
\hline 9108.98 & $4.27 \pm 0.151$ & $3.88 \pm 0.0583$ & $3.57 \pm 0.0328$ & $2.88 \pm 0.0222$ & $2.35 \pm 0.0144$ & .0117 \\
\hline 9110.05 & $4.65 \pm 0.155$ & $4.08 \pm 0.0595$ & $3.72 \pm 0.0334$ & $3.04 \pm 0.0228$ & $2.39 \pm 0.0145$ & $2.13 \pm 0.0118$ \\
\hline 9110.99 & $4.67 \pm 0.157$ & $4.11 \pm 0.0598$ & $3.74 \pm 0.0335$ & $3.11 \pm 0.0231$ & $2.42 \pm 0.0146$ & $2.13 \pm 0.0118$ \\
\hline 9111.99 & $3.94 \pm 0.150$ & $3.72 \pm 0.0572$ & $3.48 \pm 0.0324$ & $3.01 \pm 0.0227$ & $2.38 \pm 0.0144$ & $2.16 \pm 0.0119$ \\
\hline 9113.00 & $3.67 \pm 0.142$ & $3.47 \pm 0.0551$ & $3.17 \pm 0.0310$ & $2.75 \pm 0.0217$ & $2.17 \pm 0.0138$ & $1.94 \pm 0.0112$ \\
\hline 9114.00 & $3.78 \pm 0.150$ & $3.49 \pm 0.0539$ & $3.20 \pm 0.0305$ & $2.73 \pm 0.0213$ & $2.16 \pm 0.0133$ & $1.95 \pm 0.0107$ \\
\hline 9115.00 & $3.77 \pm 0.147$ & $3.56 \pm 0.0559$ & $3.29 \pm 0.0316$ & $2.77 \pm 0.0218$ & $2.13 \pm 0.0137$ & $1.94 \pm 0.0112$ \\
\hline 9116.01 & $4.05 \pm 0.146$ & $3.62 \pm 0.0541$ & $3.31 \pm 0.0307$ & $2.76 \pm 0.0210$ & $2.16 \pm 0.0131$ & $1.97 \pm 0.0106$ \\
\hline 9116.95 & $3.88 \pm 0.146$ & $3.47 \pm 0.0545$ & $3.31 \pm 0.0313$ & $2.75 \pm 0.0214$ & $2.17 \pm 0.0134$ & $1.96 \pm 0.0109$ \\
\hline 9117.88 & $3.94 \pm 0.151$ & $3.76 \pm 0.0574$ & $3.31 \pm 0.0316$ & $2.79 \pm 0.0219$ & $2.19 \pm 0.0139$ & $1.98 \pm 0.0114$ \\
\hline 9118.95 & $4.01 \pm 0.285$ & $4.01 \pm 0.0805$ & $3.53 \pm 0.0416$ & $2.94 \pm 0.0225$ & $2.37 \pm 0.0144$ & $2.17 \pm 0.0119$ \\
\hline 9119.96 & $4.17 \pm 0.218$ & $4.00 \pm 0.0747$ & $3.79 \pm 0.0406$ & $3.14 \pm 0.0232$ & $2.52 \pm 0.0149$ & $2.33 \pm 0.0123$ \\
\hline 9120.96 & $4.62 \pm 0.168$ & $4.30 \pm 0.0619$ & $3.93 \pm 0.0347$ & $3.22 \pm 0.0235$ & $2.57 \pm 0.0150$ & $2.37 \pm 0.0124$ \\
\hline 9121.97 & $4.87 \pm 0.169$ & $4.45 \pm 0.0627$ & $4.05 \pm 0.0351$ & $3.24 \pm 0.0236$ & $2.61 \pm 0.0151$ & $2.40 \pm 0.0125$ \\
\hline 9122.97 & $5.25 \pm 0.167$ & $4.62 \pm 0.0637$ & $4.12 \pm 0.0353$ & $3.29 \pm 0.0238$ & $2.58 \pm 0.0150$ & $2.31 \pm 0.0123$ \\
\hline 9123.98 & $4.95 \pm 0.165$ & $4.48 \pm 0.0628$ & $4.08 \pm 0.0352$ & $3.33 \pm 0.0239$ & $2.58 \pm 0.0150$ & $2.37 \pm 0.0124$ \\
\hline 9124.92 & $4.76 \pm 0.163$ & $4.33 \pm 0.0616$ & $3.84 \pm 0.0341$ & $3.15 \pm 0.0232$ & $2.49 \pm 0.0148$ & $2.26 \pm 0.0121$ \\
\hline 9125.92 & $4.01 \pm 0.153$ & $4.04 \pm 0.0598$ & $3.58 \pm 0.0330$ & $3.00 \pm 0.0227$ & $2.41 \pm 0.0145$ & $2.18 \pm 0.0119$ \\
\hline 9126.93 & $4.31 \pm 0.156$ & $3.86 \pm 0.0583$ & $3.52 \pm 0.0327$ & $2.92 \pm 0.0224$ & $2.32 \pm 0.0143$ & $2.10 \pm 0.0117$ \\
\hline 9127.93 & $3.92 \pm 0.148$ & $3.79 \pm 0.0576$ & $3.36 \pm 0.0319$ & $2.86 \pm 0.0222$ & $2.20 \pm 0.0139$ & $1.99 \pm 0.0114$ \\
\hline 9129.00 & $3.85 \pm 0.363$ & $3.50 \pm 0.105$ & $3.19 \pm 0.0550$ & $2.76 \pm 0.0217$ & $2.13 \pm 0.0137$ & $1.92 \pm 0.0112$ \\
\hline 9129.94 & $3.59 \pm 0.149$ & $3.41 \pm 0.0544$ & $3.03 \pm 0.0306$ & $2.59 \pm 0.0218$ & $2.08 \pm 0.0133$ & $1.89 \pm 0.0106$ \\
\hline 9130.94 & $3.46 \pm 0.144$ & $3.63 \pm 0.0566$ & $3.13 \pm 0.0308$ & $2.61 \pm 0.0208$ & $2.06 \pm 0.0132$ & $1.90 \pm 0.0108$ \\
\hline 9131.88 & $3.04 \pm 0.139$ & $3.21 \pm 0.0535$ & $2.89 \pm 0.0298$ & $2.55 \pm 0.0209$ & $2.01 \pm 0.0131$ & $1.86 \pm 0.0107$ \\
\hline 9132.95 & $3.25 \pm 0.141$ & $3.16 \pm 0.0530$ & $2.90 \pm 0.0298$ & $2.45 \pm 0.0205$ & $1.93 \pm 0.0131$ & $1.82 \pm 0.0109$ \\
\hline 9133.76 & $3.13 \pm 0.140$ & $2.95 \pm 0.0513$ & $2.64 \pm 0.0285$ & $2.40 \pm 0.0203$ & $1.93 \pm 0.0130$ & $1.78 \pm 0.0108$ \\
\hline 9135.09 & $2.73 \pm 0.131$ & $2.93 \pm 0.0510$ & $2.48 \pm 0.0276$ & $2.25 \pm 0.0197$ & $1.83 \pm 0.0127$ & $1.70 \pm 0.0105$ \\
\hline
\end{tabular}

${ }^{\text {a }}$ Rest-frame (direct-integration) flux densities, in units of $10^{-14} \mathrm{ergs} \mathrm{s}^{-1} \mathrm{~cm}^{-2} \AA^{-1}$.

$1633 \AA$. The $C$ IV wings were thus defined to lie between velocity offsets of 3000 and $10,840 \mathrm{~km} \mathrm{~s}^{-1}$. We illustrate these divisions in Figure 7. The red wing may be contaminated with very broad $\mathrm{He}$ II emission as well as possible emission from $\mathrm{Fe}$ II (e.g., $\lambda 1608$ ). The wavelength interval for the $\operatorname{Ly} \alpha$ flux was chosen to avoid geocoronal emission as well as emission from $\mathrm{N} \mathrm{V}$ and has approximately the same width as the core of C IV. The wavelength interval for $\mathrm{N} v$ was chosen to avoid most of the $\mathrm{Ly} \alpha$ core emission; however, the measured flux is missing most of its blue-side flux and is certain to lie atop the red wing of $\mathrm{Ly} \alpha$. The wavelength interval for Si IV + O IV ] was chosen to avoid the the unidentified emission near $1465 \AA$. Finally, the wavelength interval designated as $\mathrm{C} \mathrm{III]}+\mathrm{Si}$ III] contains a small contribution from Al III $\lambda 1859$, and possibly weak emission from Fe II.

The rest-frame emission-line fluxes and their uncertainties are tabulated in Table 13 for the strong lines ( $\operatorname{Ly} \alpha, \mathrm{C}$ IV $\lambda 1549$, and $\left.\mathrm{C}_{\mathrm{III}}\right] \lambda 1909+\mathrm{Si}$ III] $\lambda 1893$ ), in Table 14 for the weak lines (N v $\lambda 1240$, Si IV $\lambda 1400+\mathrm{O}$ IV ] $\lambda 1402$, and He II $\lambda 1640+$ $\mathrm{O}$ III] $\lambda 1663$ ), and in Table 15 for the C IV emission-line com- ponents. The uncertainty in the emission-line flux is the statistical one, and includes a contribution from the statistical uncertainty in the continuum placement. The uncertainty does not include the contributions from the systematic errors discussed in $\S 2$ and Appendices $C$ and $D$. These are additional sources of uncertainty whose combined amplitude is likely to be at the $2 \%-4.5 \%$ levels for most observations. All values are given in units of $10^{-14} \mathrm{ergs} \mathrm{s}^{-1} \mathrm{~cm}^{-2}$. We plot the strong and weak emission-line light curves in Figure 8, and the C IV emission-line components in Figure 9.

\subsubsection{Spectral Fitting}

The spectral fitting was done in much the same way as in Papers I and V, i.e., by using the IRAF task SPECFIT (Kriss 1994 ) to perform a multidimensional $\chi^{2}$ minimization utilizing alternating iterations of a Simplex algorithm and a Levenberg-Marquardt algorithm, with the errors properly propagated through the fit. The weakest emission lines (i.e., O I $\lambda 1302, \mathrm{C}$ II $\lambda 1335, \mathrm{~N}$ IV ] $\lambda 1486, \mathrm{~N}$ III] $\lambda 1750, \mathrm{Al}$ III $\lambda \lambda 1855$, 

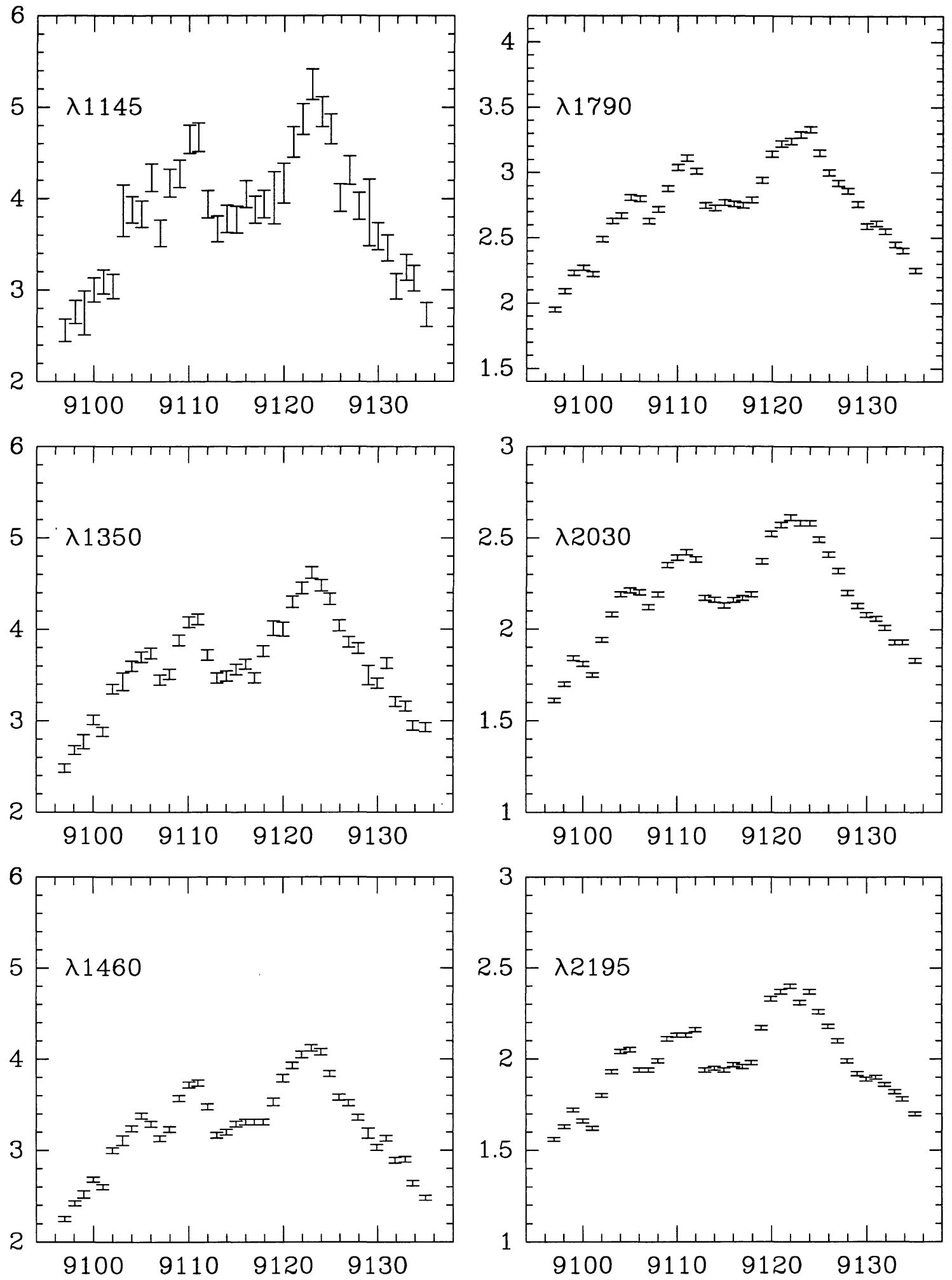

FIG. 6.-Six FOS continuum light curves, as given in Table 12. The ordinate has units of $10^{-14} \mathrm{ergs} \mathrm{s}^{-1} \mathrm{~cm}^{-2} \AA^{-1}$, and the abscissa shows Julian Date minus $2,440,000$. 


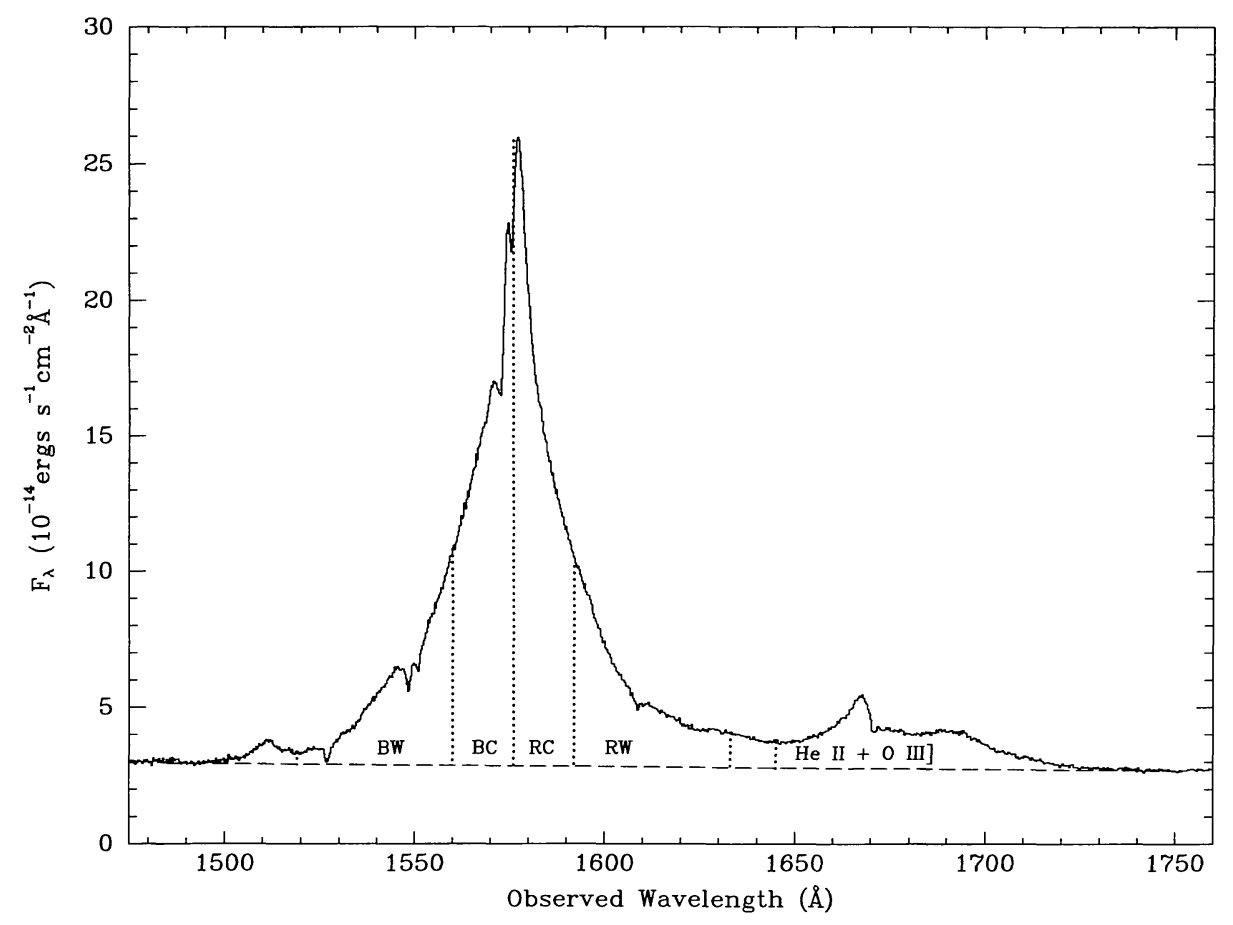

FIG. 7.-Mean FOS C IV emission-line profile, marking the divisions of the profile components: blue wing (BW), blue core (BC), red core (RC), and red wing (RW). Also shown is the designated He II $+\mathrm{O}$ III] region, and the interpolated linear continuum running through the two continuum windows straddling these emission lines.

1863, and various weak Si II and Fe II features) were modeled with single Gaussians, while the stronger emission lines (i.e., Ly $\alpha \lambda 1216$, N v $\lambda 1240$, C IV $\lambda 1549$, He II $\lambda 1640$, O III] $\lambda 1663$, Si III] $\lambda 1893$, and C III] $\lambda 1909$ ) were modeled with three Gaussians, described by narrow, broad, and very broad widths. We found that the weaker components of blended emission lines were required to be constrained by various means. For example, the widths of the broad and very broad components of He II were tied to those determined for C IV, their central wavelengths were forced to be identical (but not necessarily the same as that of the narrow component), and their flux ratio was forced to be that determined from the fit to the mean C IV profile. $\mathrm{N} v$ was forced to have a fixed profile which was determined from the mean spectrum. Galactic absorption lines were modeled with the $\mathrm{G} 130 \mathrm{H}$ and $\mathrm{G} 190 \mathrm{H}$ line-spread functions (LSFs; Evans 1993), and their equivalent widths were fixed to those values derived from the fit to the mean spectrum. The broader intrinsic blueshifted absorption lines ( Ly $\alpha, \mathrm{N} \mathrm{V}$, and C IV ) were modeled with Gaussians, and their equivalent widths were allowed to vary. We assumed that the intrinsic features absorbed continuum plus all emission components present within the absorption profile. See Paper V for more details regarding the spectral fitting technique.

The one important difference in the spectral fitting technique employed here and that employed in Papers I and $\mathrm{V}$ is that these spectra were fitted piecewise, the individual fits occurring between two continuum windows ( see $\S 5.2 .1$ ) straddling the emission lines to be fitted. Simple power-law continua, along with the aforementioned emission-line compo- nents, were fitted simultaneously in three spectral regions separately: 1155-1380 $\mathrm{A}, 1475-1760 \AA$, and 1740-2030 $\AA$. This method forces the continua to go through or near the observed data points within the continuum windows. When the emission lines and continuum were fitted simultaneously over the entire wavelength range in the spectra, the resulting fits to the continua lay well below the observed pixel values, except at the very ends of the spectra (where the systematic errors in the FOS spectra have their greatest amplitudes). The emission-line wings, in our simple parameterization of their profiles stated above, overlapped everywhere. The continuum level and shape in this fit are not well constrained by these data, considering the uncertainties in the strengths of the very broad emission-line wings and in the various possible continuum contributions (e.g., power-law, accretion disk, Balmer recombination continuum, Fe II pseudo-continuum). Thus, in the spirit of our long-standing philosophy of keeping the interpretation a minimum in papers describing the monitoring data we have obtained, a simple piecewise fitting procedure was adopted. The remaining advantages of this spectral fitting method over the direct integration are twofold, but model-dependent: the ability to "deblend" weakly blended emission lines and "remove" absorption features. More sophisticated spectral modeling is left for future work.

The mean FOS spectrum was fitted first to derive fitting templates, which were then applied as initial guesses to fitting the three pieces for each of the individual spectra. We emphasize that we ascribe no physical significance to the individual Gaussian components comprising an individual emission line. 
TABLE 13

FOS STRONG EMISSION LINES

\begin{tabular}{|c|c|c|c|c|c|c|}
\hline \multirow{2}{*}{$\begin{array}{l}\text { Julian Date } \\
(2440000+)\end{array}$} & \multicolumn{2}{|c|}{ Ly $\alpha \lambda 1216$} & \multicolumn{2}{|c|}{ C IV $\lambda 1549$} & \multicolumn{2}{|c|}{$\mathrm{C}_{\text {III] }]} \lambda 1909+\mathrm{Si}{ }_{111]} \lambda 1893$} \\
\hline & (Direct) & (Fit) & (Direct) & (Fit) & & \\
\hline 9097.00 & $332 . \pm 2.44$ & $553 . \pm 14.6$ & $554 . \pm 2.03$ & $579 . \pm 4.33$ & $116 . \pm 1.03$ & $115 . \pm 1.17$ \\
\hline 9098.07 & $326 . \pm 2.44$ & $534 . \pm 15.0$ & $541 . \pm 2.04$ & $568 . \pm 4.29$ & $114 . \pm 1.05$ & $115 . \pm 1.29$ \\
\hline 9099.01 & $325 . \pm 3.94$ & $592 . \pm 17.7$ & $558 . \pm 2.72$ & $573 . \pm 5.50$ & $113 . \pm 1.08$ & $117 . \pm 1.24$ \\
\hline 9100.01 & $333 . \pm 2.49$ & $562 . \pm 17.2$ & $564 . \pm 2.10$ & $596 . \pm 4.39$ & $111 . \pm 1.08$ & $115 . \pm 1.28$ \\
\hline 9101.02 & $322 . \pm 2.46$ & $536 . \pm 16.5$ & $569 . \pm 2.10$ & $600 . \pm 4.53$ & $109 . \pm 1.07$ & $111 . \pm 1.34$ \\
\hline 9102.02 & $353 . \pm 2.56$ & $623 . \pm 17.1$ & $572 . \pm 2.16$ & $598 . \pm 5.15$ & $109 . \pm 1.11$ & $112 . \pm 1.40$ \\
\hline 9103.09 & $342 . \pm 4.59$ & $630 . \pm 25.3$ & $598 . \pm 3.16$ & $614 . \pm 7.60$ & $114 . \pm 1.15$ & $117 . \pm 1.43$ \\
\hline 9104.03 & $368 . \pm 2.65$ & $657 . \pm 23.9$ & $612 . \pm 2.24$ & $642 . \pm 5.60$ & $119 . \pm 1.16$ & $119 . \pm 1.50$ \\
\hline 9105.03 & $370 . \pm 2.66$ & $618 . \pm 15.2$ & $612 . \pm 2.26$ & $644 . \pm 4.34$ & $118 . \pm 1.18$ & $119 . \pm 1.57$ \\
\hline 9106.03 & $363 . \pm 2.66$ & $609 . \pm 30.4$ & $633 . \pm 2.27$ & $658 . \pm 4.48$ & $116 . \pm 1.17$ & $117 . \pm 1.52$ \\
\hline 9106.97 & $385 . \pm 2.68$ & $660 . \pm 16.5$ & $652 . \pm 2.27$ & $684 . \pm 4.73$ & $120 . \pm 1.15$ & $119 . \pm 1.66$ \\
\hline 9107.98 & $388 . \pm 2.72$ & $658 . \pm 15.0$ & $654 . \pm 2.29$ & $689 . \pm 4.92$ & $119 . \pm 1.17$ & $122 . \pm 1.54$ \\
\hline 9108.98 & $399 . \pm 2.76$ & $686 . \pm 18.3$ & $657 . \pm 2.33$ & $687 . \pm 4.63$ & $128 . \pm 1.20$ & $123 . \pm 1.53$ \\
\hline 9110.05 & $394 . \pm 2.77$ & $672 . \pm 19.9$ & $658 . \pm 2.35$ & $685 . \pm 4.50$ & $123 . \pm 1.22$ & $125 . \pm 1.63$ \\
\hline 9110.99 & $382 . \pm 2.82$ & $666 . \pm 19.9$ & $659 . \pm 2.36$ & $688 . \pm 4.62$ & $118 . \pm 1.22$ & $119 . \pm 1.47$ \\
\hline 9111.99 & $394 . \pm 2.81$ & $702 . \pm 17.0$ & $670 . \pm 2.34$ & $699 . \pm 5.20$ & $121 . \pm 1.21$ & $124 . \pm 1.64$ \\
\hline 9113.00 & $387 . \pm 2.76$ & $688 . \pm 20.3$ & $669 . \pm 2.30$ & $698 . \pm 4.96$ & $122 . \pm 1.17$ & $121 . \pm 1.43$ \\
\hline 9114.00 & $408 . \pm 2.72$ & $739 . \pm 21.6$ & $670 . \pm 2.26$ & $698 . \pm 5.70$ & $126 . \pm 1.15$ & $124 . \pm 1.49$ \\
\hline 9115.00 & $393 . \pm 2.79$ & $735 . \pm 15.4$ & $673 . \pm 2.32$ & $703 . \pm 5.24$ & $126 . \pm 1.18$ & $126 . \pm 1.71$ \\
\hline 9116.01 & $399 . \pm 2.70$ & $678 . \pm 22.2$ & $683 . \pm 2.27$ & $719 . \pm 5.04$ & $121 . \pm 1.13$ & $121 . \pm 1.45$ \\
\hline 9116.95 & $419 . \pm 2.79$ & $766 . \pm 22.2$ & $689 . \pm 2.31$ & $720 . \pm 4.96$ & $131 . \pm 1.16$ & $130 . \pm 1.43$ \\
\hline 9117.88 & $434 . \pm 2.92$ & $798 . \pm 16.7$ & $701 . \pm 2.35$ & $734 . \pm 5.05$ & $127 . \pm 1.19$ & $128 . \pm 1.56$ \\
\hline 9118.95 & $433 . \pm 4.18$ & $792 . \pm 29.5$ & $709 . \pm 2.74$ & $739 . \pm 6.08$ & $132 . \pm 1.22$ & $131 . \pm 1.50$ \\
\hline 9119.96 & $429 . \pm 3.88$ & $759 . \pm 28.2$ & $717 . \pm 2.73$ & $743 . \pm 4.91$ & $133 . \pm 1.25$ & $132 . \pm 1.53$ \\
\hline 9120.96 & $461 . \pm 3.05$ & $784 . \pm 26.9$ & $704 . \pm 2.43$ & $729 . \pm 4.58$ & $137 . \pm 1.26$ & $138 . \pm 1.64$ \\
\hline 9121.97 & $462 . \pm 3.07$ & $810 . \pm 17.3$ & $719 . \pm 2.46$ & $750 . \pm 4.72$ & $141 . \pm 1.28$ & $138 . \pm 1.63$ \\
\hline 9122.97 & $445 . \pm 3.02$ & $736 . \pm 21.9$ & $724 . \pm 2.47$ & $755 . \pm 4.54$ & $136 . \pm 1.27$ & $135 . \pm 1.68$ \\
\hline 9123.98 & $465 . \pm 3.06$ & $749 . \pm 22.8$ & $730 . \pm 2.47$ & $771 . \pm 5.43$ & $137 . \pm 1.27$ & $136 . \pm 1.42$ \\
\hline 9124.92 & $465 . \pm 3.05$ & $804 . \pm 16.9$ & $740 . \pm 2.46$ & $769 . \pm 5.14$ & $140 . \pm 1.26$ & $137 . \pm 1.67$ \\
\hline 9125.92 & $458 . \pm 2.99$ & $805 . \pm 18.4$ & $735 . \pm 2.42$ & $760 . \pm 4.43$ & $143 . \pm 1.24$ & $140 . \pm 1.72$ \\
\hline 9126.93 & $462 . \pm 3.01$ & $795 . \pm 20.9$ & $739 . \pm 2.41$ & $768 . \pm 4.95$ & $138 . \pm 1.22$ & $137 . \pm 1.52$ \\
\hline 9127.93 & 457.土2.97 & $819 . \pm 19.0$ & $732 . \pm 2.39$ & $760 . \pm 4.16$ & $136 . \pm 1.20$ & $136 . \pm 1.53$ \\
\hline 9129.00 & $480 . \pm 5.96$ & $834 . \pm 43.5$ & $728 . \pm 3.64$ & $742 . \pm 6.59$ & $135 . \pm 1.19$ & $137 . \pm 1.54$ \\
\hline 9129.94 & $453 . \pm 2.89$ & $821 . \pm 15.2$ & $724 . \pm 2.40$ & $754 . \pm 5.04$ & $137 . \pm 1.19$ & $134 . \pm 1.54$ \\
\hline 9130.94 & $467 . \pm 2.97$ & $808 . \pm 15.3$ & $725 . \pm 2.34$ & $757 . \pm 5.12$ & $141 . \pm 1.15$ & $140 . \pm 1.61$ \\
\hline 9131.88 & $480 . \pm 2.97$ & $862 . \pm 13.9$ & $741 . \pm 2.34$ & $774 . \pm 5.13$ & $143 . \pm 1.16$ & $141 . \pm 1.46$ \\
\hline 9132.95 & $471 . \pm 2.96$ & $834 . \pm 28.5$ & $718 . \pm 2.31$ & 757.土5.02 & $138 . \pm 1.15$ & $139 . \pm 1.40$ \\
\hline 9133.76 & $459 . \pm 2.92$ & $830 . \pm 39.5$ & $720 . \pm 2.29$ & $754 . \pm 5.02$ & $144 . \pm 1.14$ & $145 . \pm 1.48$ \\
\hline 9135.09 & $443 . \pm 2.85$ & $796 . \pm 18.5$ & $706 . \pm 2.26$ & $740 . \pm 5.26$ & $143 . \pm 1.12$ & $142 . \pm 1.49$ \\
\hline
\end{tabular}

${ }^{\text {a }}$ Rest-frame fluxes in units of $10^{-14} \mathrm{ergs} \mathrm{s}^{-1} \mathrm{~cm}^{-2}$.

They merely represent a convenient way of parameterizing the line profile in order to measure the line flux. This may also be said of two classes of severely blended emission lines: (1) weak emission lying atop much stronger emission (e.g., $\mathrm{N} v \lambda 1240$ ) and (2) two emission lines of comparable flux (e.g., C III] $\lambda 1909+\mathrm{Si}$ III $] \lambda 1893)$. While every effort was made to deblend these lines, no such attempt was made for the Si IV $\lambda 1400+$ O IV ] $\lambda 1402$ complex. We present here the results of the fits to the two strongest emission lines, least affected by blending ( $\operatorname{Ly} \alpha, C$ IV and its profile components), and also that of the blended emission of $\mathrm{He}$ II $+\mathrm{O}$ III]. Although weak, the He II + $\mathrm{O}$ III] blend is sufficiently well separated from $\mathrm{C}$ IV to derive a meaningful light curve. We also present the fitted fluxes of the summed emission from the C III] $\lambda 1909+\mathrm{Si}$ III] $\lambda 1893$ blend, which includes a small contribution from $\mathrm{Al}$ III $\lambda 1859$, in order to show the comparison between the two methods of measuring the emission-line fluxes when they are measuring the same emission-line features. Notice that in this case the blue-side continuum window used for interpolation under the emission feature differed from that used in the direct integration.

The fluxes derived from the fits are tabulated next to the corresponding direct-integration values in Tables 13-15. The fitted emission-line fluxes are the total fluxes, integrated over the sum of the Gaussian components which have been fitted to these emission lines. In Figure 8 we show the fitted flux only for the total Ly $\alpha$ emission line, because it was the only tabulated fitted emission-line component whose light curve differed significantly from its corresponding direct-integration light curve. In contrast to the sum of the fitted components (labeled "Ly $\alpha$ total" in Fig. 8), the direct integration of $\operatorname{Ly} \alpha$ (labeled "Ly $\alpha$ core") did not include the wings and did not compensate for significant intrinsic Ly $\alpha$ absorption in its core. We note that the fit to the $\mathrm{N} v$ emission line gave results which were consistent with, but noisier than, the direct integration. For the purpose of comparison, in Figure 9 we show the fitted results of the C IV components plotted on top of the corresponding direct 
TABLE 14

FOS WEAK EMISSION LINES ${ }^{\mathrm{a}}$

\begin{tabular}{ccccc} 
Julian Date & N v $\lambda 1240$ & Si IV $\lambda 1400+$ O IV $]$ & $\lambda 1402$ & He II $\lambda 1640+$ O III $\lambda 1663$ \\
$(2440000+)$ & (Direct) & (Direct) & (Direct) & (Fit) \\
& & & & \\
\hline 9097.00 & $57.8 \pm 1.40$ & $59.1 \pm 1.27$ & $63.0 \pm 1.13$ & $70.8 \pm 2.96$ \\
9098.07 & $60.8 \pm 1.44$ & $56.4 \pm 1.30$ & $59.3 \pm 1.16$ & $68.7 \pm 3.27$ \\
9099.01 & $71.6 \pm 2.45$ & $59.3 \pm 2.01$ & $65.4 \pm 1.21$ & $76.0 \pm 4.21$ \\
9100.01 & $66.2 \pm 1.51$ & $56.4 \pm 1.36$ & $66.0 \pm 1.20$ & $78.4 \pm 3.22$ \\
9101.02 & $62.1 \pm 1.49$ & $62.4 \pm 1.35$ & $65.9 \pm 1.20$ & $78.2 \pm 4.03$ \\
9102.02 & $78.5 \pm 1.58$ & $62.8 \pm 1.43$ & $72.0 \pm 1.27$ & $83.3 \pm 4.34$ \\
9103.09 & $72.2 \pm 2.96$ & $73.4 \pm 2.54$ & $78.3 \pm 1.34$ & $88.9 \pm 4.98$ \\
9104.03 & $75.7 \pm 1.66$ & $69.4 \pm 1.49$ & $82.3 \pm 1.34$ & $92.4 \pm 3.44$ \\
9105.03 & $83.7 \pm 1.69$ & $69.4 \pm 1.51$ & $82.0 \pm 1.36$ & $93.2 \pm 4.15$ \\
9106.03 & $73.5 \pm 1.68$ & $67.0 \pm 1.50$ & $90.8 \pm 1.37$ & $105 . \pm 4.08$ \\
9106.97 & $80.9 \pm 1.65$ & $74.1 \pm 1.48$ & $91.8 \pm 1.34$ & $107 . \pm 3.96$ \\
9107.98 & $73.7 \pm 1.67$ & $77.3 \pm 1.50$ & $88.6 \pm 1.35$ & $101 . \pm 4.93$ \\
9108.98 & $83.9 \pm 1.73$ & $74.4 \pm 1.55$ & $89.5 \pm 1.40$ & $104 . \pm 3.61$ \\
9110.05 & $80.0 \pm 1.76$ & $75.4 \pm 1.58$ & $90.5 \pm 1.42$ & $102 . \pm 4.82$ \\
9110.99 & $78.0 \pm 1.76$ & $74.7 \pm 1.58$ & $91.9 \pm 1.43$ & $107 . \pm 4.12$ \\
9111.99 & $88.5 \pm 1.72$ & $79.8 \pm 1.54$ & $98.0 \pm 1.42$ & $111 . \pm 4.04$ \\
9113.00 & $77.6 \pm 1.64$ & $77.1 \pm 1.49$ & $93.6 \pm 1.37$ & $109 . \pm 4.71$ \\
9114.00 & $74.1 \pm 1.58$ & $80.1 \pm 1.47$ & $91.9 \pm 1.35$ & $107 . \pm 3.96$ \\
9115.00 & $76.2 \pm 1.66$ & $76.9 \pm 1.51$ & $89.1 \pm 1.37$ & $102 . \pm 4.33$ \\
9116.01 & $79.3 \pm 1.62$ & $76.6 \pm 1.46$ & $89.2 \pm 1.33$ & $102 . \pm 3.93$ \\
9116.95 & $80.7 \pm 1.64$ & $82.7 \pm 1.49$ & $91.5 \pm 1.36$ & $106 . \pm 4.25$ \\
9117.88 & $78.3 \pm 1.69$ & $76.6 \pm 1.53$ & $90.9 \pm 1.38$ & $107 . \pm 4.07$ \\
9118.95 & $85.4 \pm 2.54$ & $80.7 \pm 2.09$ & $92.2 \pm 1.42$ & $108 . \pm 4.24$ \\
9119.96 & $94.0 \pm 2.36$ & $79.0 \pm 1.96$ & $99.4 \pm 1.46$ & $114 . \pm 4.10$ \\
9120.96 & $97.0 \pm 1.85$ & $84.0 \pm 1.65$ & $98.4 \pm 1.48$ & $116 . \pm 4.38$ \\
9121.97 & $95.2 \pm 1.87$ & $82.9 \pm 1.66$ & $106 . \pm 1.50$ & $124 . \pm 4.24$ \\
9122.97 & $92.6 \pm 1.88$ & $87.2 \pm 1.68$ & $105 . \pm 1.51$ & $120 . \pm 5.25$ \\
9123.98 & $101 . \pm 1.88$ & $93.0 \pm 1.68$ & $106 . \pm 1.50$ & $118 . \pm 3.85$ \\
9124.92 & $98.7 \pm 1.85$ & $90.2 \pm 1.65$ & $107 . \pm 1.48$ & $117 . \pm 3.85$ \\
9125.92 & $96.4 \pm 1.78$ & $85.7 \pm 1.60$ & $108 . \pm 1.45$ & $122 . \pm 3.40$ \\
9126.93 & $90.9 \pm 1.77$ & $89.3 \pm 1.58$ & $103 . \pm 1.42$ & $120 . \pm 3.77$ \\
9127.93 & $90.4 \pm 1.73$ & $90.0 \pm 1.56$ & $99.7 \pm 1.40$ & $114 . \pm 3.71$ \\
9129.00 & $100 . \pm 3.50$ & $91.0 \pm 2.80$ & $98.9 \pm 1.41$ & $118 . \pm 5.05$ \\
9129.94 & $85.0 \pm 1.64$ & $86.0 \pm 1.49$ & $94.8 \pm 1.39$ & $108 . \pm 3.86$ \\
9130.94 & $88.2 \pm 1.68$ & $79.9 \pm 1.51$ & $93.7 \pm 1.32$ & $105 . \pm 3.62$ \\
9131.88 & $95.4 \pm 1.65$ & $92.7 \pm 1.49$ & $94.8 \pm 1.32$ & $108 . \pm 4.22$ \\
9132.95 & $84.4 \pm 1.62$ & $86.2 \pm 1.46$ & $86.7 \pm 1.30$ & $99.8 \pm 3.63$ \\
9133.76 & $82.0 \pm 1.59$ & $90.2 \pm 1.44$ & $87.1 \pm 1.27$ & $97.3 \pm 3.54$ \\
9135.09 & $74.4 \pm 1.52$ & $77.4 \pm 1.39$ & $80.2 \pm 1.23$ & $91.4 \pm 3.15$ \\
& & & &
\end{tabular}

${ }^{\mathrm{a}}$ Rest-frame fluxes in units of $10^{-14} \mathrm{ergs} \mathrm{s}^{-1} \mathrm{~cm}^{-2}$.

integrations. The two separate red-core measurements are virtually identical, and the small differences in the other components are easily understood. The differences between the two blue-wing measurements arise because the fitted version extends to higher radial velocity and corrects for a small amount of Galactic absorption. The intrinsic absorption in the blue core accounts for the differences there. In the red wing the fit removed a small amount of possible contaminating emission, but otherwise the two light curves for the red wing are nearly identical.

Finally, based upon these fits, the intrinsic absorption lines had roughly constant equivalent widths ( that of C IV was approximately $0.8 \AA$ ). Together with the Galactic absorption, they constituted from $\sim 2 \%$ to $\sim 10 \%$ of the flux in any of the emission-line measurements tabulated here, with the exception of $\operatorname{Ly} \alpha$. In this case, the absorbed line flux amounted to $\sim 25 \%$ of the directly measured core flux and $\sim 15 \%$ of the fitted total Ly $\alpha$ flux. We note here that the intrinsic absorption is apparently much weaker than that found in the 1988-1989 campaign for C IV by Shull \& Sachs (1993), but we defer more detailed analyses of the intrinsic absorption to later work.

\subsection{The IUE SWP Spectral Measurements}

Before making measurements, the SWP spectra were shifted in wavelength so as to produce alignment with the peak of the $\mathrm{C}$ IV emission line, whose position is based upon a smoothed mean FOS spectrum. Emission-line and continuum fluxes were measured using the direct-integration and fitting techniques discussed above. The major difference in the direct-integration technique applied to the SWP spectra was that broader, $40 \AA$ continuum windows were used. The major difference in fitting the SWP spectra was that, as in Papers I and $\mathrm{V}$, the full SWP spectrum was fitted, with components based upon the results of the fit to the mean FOS spectrum. The weakest lines were omitted from or held fixed during the spectral fitting, and absorption lines were modeled with Gaussians whose fixed widths corresponded to the IUE spectral resolution. The direct integrations of the $\mathrm{C} \mathrm{III]} \mathrm{and} \mathrm{Ly} \alpha+$ $\mathrm{N} v$ regions subtracted a constant value in continuum flux $\left(F_{\lambda}\right)$, based upon the value derived in the nearest continuum window. Continuum windows on both sides of these two regions were not available for a linear interpolation.

The error bars on the SWP measurements were evaluated as explained in detail in Paper V. As in Paper V, the statistical error bars on the SWP pixel values were found to be too small, and a $\chi^{2}$ analysis has shown that each NEWSIPS pixel is probably correlated with approximately two neighboring pixels. The NEWSIPS error bars on the pixel values were, therefore, increased by a factor equal to the square root of the number of correlated pixels, $\sim 1.6$. Next, the measurement error bars were evaluated. Unlike the case in Paper V, contemporaneous pairs of SWP spectra were not available. Instead, in the 20 instances where the SWP and FOS observations were contemporaneous, the median value of the ratio (FOS measurement)/ (SWP measurement) was computed. After temporarily scaling the SWP measurement by this ratio, a distribution of the following quantity was found: $\left(F_{\mathrm{SWP}}-F_{\mathrm{FOS}}\right)^{2} /\left(\sigma_{\mathrm{SWP}}^{2}+\sigma_{\mathrm{FOS}}^{2}\right)$. For Gaussian errors this should look like a $\chi^{2}$ distribution peaking near 1 , if the error bars on the measured flux values are the correct size. We found their distributions were approximately $\chi^{2}$, but their peaks were generally greater than unity ( $\sim 2$ for the well-measured values). The measurement error bars were scaled by the square root of the median value of their distributions. The actual flux measurements reported here were left unchanged.

The SWP continuum and emission-line measurements are listed in Tables 16 and 17 and are plotted in Figures 10 and 11, respectively. The Julian Dates associated with the SWP measurements are those at the start of the exposure. We present in these tables and figures the fitted measurements only, with the exception of the $\mathrm{N} v \lambda 1240$ fluxes which were derived from direct integration, described above; in the case of the $\mathrm{N} \mathrm{v}$ fluxes, the direct-integration measurements are used because the fitted fluxes are not well constrained in the relatively low signal-to-noise IUE spectra and thus show considerable scatter. We found that the fitting procedure minimized the effects 
TABLE 15

FOS C IV $\lambda 1549$ COMPONENTS $^{\mathrm{a}}$

\begin{tabular}{|c|c|c|c|c|c|c|c|c|}
\hline \multirow{2}{*}{$\begin{array}{l}\text { Julian Date } \\
(2440000+)\end{array}$} & \multicolumn{2}{|c|}{ Blue Wing } & \multicolumn{2}{|c|}{ Blue Core } & \multicolumn{2}{|c|}{ Red Core } & \multicolumn{2}{|c|}{ Red Wing } \\
\hline & (Direct) & (Fit) & (Direct) & (Fit) & (Direct) & (Fit) & (Direct) & (Fit) \\
\hline 9097.00 & $98.3 \pm 0.985$ & $110 . \pm 0.822$ & $184 . \pm 1.06$ & $203 . \pm 1.51$ & $180 . \pm 0.981$ & $182 . \pm 1.37$ & $91.1 \pm 1.04$ & $84.2 \pm 0.629$ \\
\hline 9098.07 & $94.0 \pm 0.993$ & $105 . \pm 0.789$ & $175 . \pm 1.04$ & $191 . \pm 1.45$ & $179 . \pm 0.986$ & $182 . \pm 1.38$ & $92.2 \pm 1.06$ & $89.1 \pm 0.672$ \\
\hline 9099.01 & $96.0 \pm 1.43$ & $107 . \pm 1.02$ & $177 . \pm 1.46$ & $183 . \pm 1.76$ & $185 . \pm 1.39$ & $186 . \pm 1.79$ & $99.6 \pm 1.14$ & $97.6 \pm 0.937$ \\
\hline 9100.01 & $97.8 \pm 1.03$ & $109 . \pm 0.800$ & $182 . \pm 1.07$ & $203 . \pm 1.49$ & $187 . \pm 1.01$ & $190 . \pm 1.40$ & $98.3 \pm 1.10$ & $94.3 \pm 0.695$ \\
\hline 9101.02 & $98.7 \pm 1.02$ & $110 . \pm 0.832$ & $178 . \pm 1.06$ & $197 . \pm 1.49$ & $190 . \pm 1.02$ & $195 . \pm 1.47$ & $103 . \pm 1.11$ & $99.3 \pm 0.749$ \\
\hline 9102.02 & $97.3 \pm 1.06$ & $110 . \pm 0.940$ & $181 . \pm 1.08$ & $195 . \pm 1.68$ & $193 . \pm 1.04$ & $197 . \pm 1.69$ & $100 . \pm 1.14$ & $97.7 \pm 0.842$ \\
\hline 9103.09 & $102 . \pm 1.72$ & $112 . \pm 1.39$ & $191 . \pm 1.72$ & $197 . \pm 2.44$ & $196 . \pm 1.60$ & $200 . \pm 2.46$ & $109 . \pm 1.24$ & $106 . \pm 1.30$ \\
\hline 9104.03 & $104 . \pm 1.10$ & $115 . \pm 1.00$ & $192 . \pm 1.11$ & $213 . \pm 1.86$ & $204 . \pm 1.07$ & $207 . \pm 1.80$ & $113 . \pm 1.20$ & $108 . \pm 0.937$ \\
\hline 9105.03 & $99.3 \pm 1.10$ & $112 . \pm 0.755$ & $193 . \pm 1.12$ & $212 . \pm 1.43$ & $205 . \pm 1.08$ & $210 . \pm 1.41$ & $114 . \pm 1.21$ & $111 . \pm 0.742$ \\
\hline 9106.03 & $107 . \pm 1.11$ & $118 . \pm 0.802$ & $199 . \pm 1.13$ & $216 . \pm 1.47$ & $207 . \pm 1.08$ & $210 \pm 1.43$ & $120 . \pm 1.22$ & $114 . \pm 0.774$ \\
\hline 9106.97 & $110 . \pm 1.10$ & $122 . \pm 0.847$ & $203 . \pm 1.13$ & $224 . \pm 1.54$ & $218 . \pm 1.09$ & $224 . \pm 1.54$ & $122 . \pm 1.21$ & $115 . \pm 0.791$ \\
\hline 9107.98 & $107 . \pm 1.11$ & $118 . \pm 0.841$ & $204 . \pm 1.14$ & $229 . \pm 1.63$ & $222 . \pm 1.11$ & $227 . \pm 1.61$ & $121 . \pm 1.22$ & $117 . \pm 0.830$ \\
\hline 9108.98 & $105 . \pm 1.13$ & $116 . \pm 0.784$ & $208 . \pm 1.16$ & $230 . \pm 1.55$ & $223 . \pm 1.12$ & $228 . \pm 1.53$ & $120 . \pm 1.24$ & $114 . \pm 0.764$ \\
\hline 9110.05 & $107 . \pm 1.15$ & $118 . \pm 0.778$ & $208 . \pm 1.16$ & $227 . \pm 1.49$ & $221 . \pm 1.12$ & $226 . \pm 1.48$ & $122 . \pm 1.26$ & $115 . \pm 0.753$ \\
\hline 9110.99 & $110 . \pm 1.16$ & $123 . \pm 0.828$ & $207 . \pm 1.16$ & $227 . \pm 1.52$ & $219 . \pm 1.12$ & $223 . \pm 1.49$ & $122 . \pm 1.27$ & $116 . \pm 0.776$ \\
\hline 9111.99 & $108 . \pm 1.13$ & $119 . \pm 0.886$ & $210 . \pm 1.16$ & $233 . \pm 1.73$ & $223 . \pm 1.12$ & $227 . \pm 1.69$ & $128 . \pm 1.26$ & $120 . \pm 0.894$ \\
\hline 9113.00 & $113 . \pm 1.11$ & $124 . \pm 0.883$ & $207 . \pm 1.15$ & $229 . \pm 1.63$ & $224 . \pm 1.11$ & $229 . \pm 1.63$ & $125 . \pm 1.23$ & $116 . \pm 0.826$ \\
\hline 9114.00 & $112 . \pm 1.09$ & $123 . \pm 1.01$ & $210 . \pm 1.13$ & $231 . \pm 1.89$ & $226 . \pm 1.09$ & $230 . \pm 1.87$ & $122 . \pm 1.20$ & $114 . \pm 0.928$ \\
\hline 9115.00 & $114 . \pm 1.13$ & $126 . \pm 0.938$ & $213 . \pm 1.16$ & $235 . \pm 1.75$ & $224 . \pm 1.11$ & $228 . \pm 1.70$ & $122 . \pm 1.23$ & $114 . \pm 0.851$ \\
\hline 9116.01 & $118 . \pm 1.11$ & $130 . \pm 0.912$ & $215 . \pm 1.14$ & $243 . \pm 1.70$ & $229 . \pm 1.10$ & $234 . \pm 1.64$ & $121 . \pm 1.19$ & $114 . \pm 0.794$ \\
\hline 9116.95 & $118 . \pm 1.12$ & $129 . \pm 0.894$ & $218 . \pm 1.16$ & $240 . \pm 1.66$ & $232 . \pm 1.11$ & $236 . \pm 1.63$ & $122 . \pm 1.21$ & $115 . \pm 0.788$ \\
\hline 9117.88 & $119 . \pm 1.14$ & $130 . \pm 0.895$ & $220 . \pm 1.18$ & $246 . \pm 1.70$ & $239 . \pm 1.15$ & $242 . \pm 1.67$ & $123 . \pm 1.23$ & $115 . \pm 0.791$ \\
\hline 9118.95 & $121 . \pm 1.41$ & $132 . \pm 1.09$ & $223 . \pm 1.42$ & $242 . \pm 1.99$ & $242 . \pm 1.37$ & $246 . \pm 2.03$ & $124 . \pm 1.28$ & $117 . \pm 0.966$ \\
\hline 9119.96 & $119 . \pm 1.39$ & $131 . \pm 0.869$ & $228 . \pm 1.42$ & $245 . \pm 1.63$ & $243 . \pm 1.36$ & $248 . \pm 1.65$ & $127 . \pm 1.30$ & $117 . \pm 0.776$ \\
\hline 9120.96 & $114 . \pm 1.19$ & $126 . \pm 0.791$ & $228 . \pm 1.22$ & $245 . \pm 1.54$ & $241 . \pm 1.17$ & $243 . \pm 1.53$ & $121 . \pm 1.28$ & $115 . \pm 0.718$ \\
\hline 9121.97 & $114 . \pm 1.20$ & $126 . \pm 0.792$ & $229 . \pm 1.23$ & $253 . \pm 1.58$ & $248 . \pm 1.18$ & $253 . \pm 1.59$ & $128 . \pm 1.31$ & $120 . \pm 0.754$ \\
\hline 9122.97 & $118 . \pm 1.21$ & $129 . \pm 0.782$ & $232 . \pm 1.23$ & $256 . \pm 1.54$ & $246 . \pm 1.18$ & $249 . \pm 1.50$ & $128 . \pm 1.31$ & $119 . \pm 0.718$ \\
\hline 9123.98 & $118 . \pm 1.21$ & $130 . \pm 0.917$ & $233 . \pm 1.23$ & $266 . \pm 1.87$ & $249 . \pm 1.19$ & $254 . \pm 1.79$ & $130 . \pm 1.31$ & $121 . \pm 0.853$ \\
\hline 9124.92 & $121 . \pm 1.19$ & $131 . \pm 0.880$ & $237 . \pm 1.23$ & $262 . \pm 1.75$ & $251 . \pm 1.18$ & $254 . \pm 1.70$ & $132 . \pm 1.30$ & $122 . \pm 0.816$ \\
\hline 9125.92 & $119 . \pm 1.17$ & $130 . \pm 0.762$ & $231 . \pm 1.21$ & $250 . \pm 1.46$ & $246 . \pm 1.17$ & $250 . \pm 1.46$ & $139 . \pm 1.29$ & $129 . \pm 0.753$ \\
\hline 9126.93 & $122 . \pm 1.17$ & $134 . \pm 0.862$ & $236 . \pm 1.22$ & $257 . \pm 1.66$ & $249 . \pm 1.17$ & $252 . \pm 1.63$ & $132 . \pm 1.27$ & $125 . \pm 0.809$ \\
\hline 9127.93 & $122 . \pm 1.15$ & $134 . \pm 0.730$ & $232 . \pm 1.21$ & $254 . \pm 1.39$ & $246 . \pm 1.16$ & $249 . \pm 1.37$ & $131 . \pm 1.26$ & $124 . \pm 0.680$ \\
\hline 9129.00 & $118 . \pm 1.91$ & $129 . \pm 1.15$ & $236 . \pm 2.03$ & $240 . \pm 2.13$ & $244 . \pm 1.91$ & $247 . \pm 2.20$ & $130 . \pm 1.35$ & $125 . \pm 1.12$ \\
\hline 9129.94 & $117 . \pm 1.13$ & $127 . \pm 0.851$ & $228 . \pm 1.22$ & $252 . \pm 1.68$ & $247 . \pm 1.18$ & $250 . \pm 1.68$ & $132 . \pm 1.27$ & $125 . \pm 0.837$ \\
\hline 9130.94 & $119 . \pm 1.12$ & $130 . \pm 0.882$ & $229 . \pm 1.19$ & $252 . \pm 1.71$ & $250 . \pm 1.16$ & $254 . \pm 1.72$ & $127 . \pm 1.20$ & $120 . \pm 0.818$ \\
\hline 9131.88 & $122 . \pm 1.11$ & $132 . \pm 0.879$ & $234 . \pm 1.19$ & $258 . \pm 1.71$ & $252 . \pm 1.16$ & $256 . \pm 1.70$ & $134 . \pm 1.21$ & $128 . \pm 0.851$ \\
\hline 9132.95 & $118 . \pm 1.10$ & $128 . \pm 0.853$ & $228 . \pm 1.18$ & $256 . \pm 1.70$ & $245 . \pm 1.14$ & $250 . \pm 1.67$ & $127 . \pm 1.20$ & $122 . \pm 0.812$ \\
\hline 9133.76 & $119 . \pm 1.08$ & $127 . \pm 0.849$ & $229 . \pm 1.18$ & $255 . \pm 1.70$ & $244 . \pm 1.14$ & $247 . \pm 1.65$ & $128 . \pm 1.18$ & $124 . \pm 0.829$ \\
\hline 9135.09 & $118 . \pm 1.06$ & $128 . \pm 0.910$ & $225 . \pm 1.16$ & $246 . \pm 1.75$ & $238 . \pm 1.12$ & $243 . \pm 1.73$ & $125 . \pm 1.16$ & $123 . \pm 0.874$ \\
\hline
\end{tabular}

${ }^{a}$ Rest-frame fluxes in units of $10^{-14} \mathrm{ergs} \mathrm{s}^{-1} \mathrm{~cm}^{-2}$.

of some of the localized artifacts, localized abnormally low fluxes, and cosmic rays prevalent with this SWP data set. We note that in most cases the fitted power-law continua went through many of the data points which lay within the three SWP continuum windows: 1350,1460 , and $1790 \AA$.

In Figure 10 we overplot in heavy symbols the corresponding FOS continuum light curves. Note the close correspondence of these measurements. The SWP recorded every major feature of the continuum variability of the $H S T$ campaign, confirming their reality. In the lower right-hand corner of each panel in Figure 10 we show the median flux ratio $F_{\text {FOS }} / F_{\text {SwP }}$ and its dispersion determined from the error-bar analysis discussed above. Most of the differences between these light curves are accounted for in the ratios given or from a perusal of Figure 5. The closest match occurs in the $1350 \AA$ continuum band, as expected. We note that the $1460 \AA$ Aight curves match somewhat better than Figure 5 would indicate because the power-law continuum fit usually fell beneath the artifact features often present in the SWP spectra within this continuum window. In Figure 11 we overplot in heavy symbols the appropriate (i.e., fitted) FOS emission-line measurements. In some cases, there is a correspondence between the differences in FOS and SWP light curves and the differences seen in the mean spectral comparisons shown in Figure 5. However, there are many instances where the differences cannot be due entirely to mean instrumental differences. Many of the single-day outliers in Figures 10 and 11 are noted in $\S 3.3$ and in Table $2 \mathrm{~A}$ as spectra with suspected problems. They were noted as such upon visual inspection of and comparison among the individual SWP spectra before the measurements were made. One such outlier, JD 2,449,071, falls off the plot of the SWP He II $+\mathrm{O}$ III] light curve in Figure 11, lying well above the points shown. The scatter in the $\mathrm{C}$ III] $+\mathrm{Si}$ III] light curve is indicative of the quality of this portion of the SWP spectra.

Taken in combination, Figures 5,10 , and 11 quantitatively illustrate (1) the mean instrumental differences remaining between the FOS and SWP and (2) the amplitude of systematic errors present in this SWP data set. 

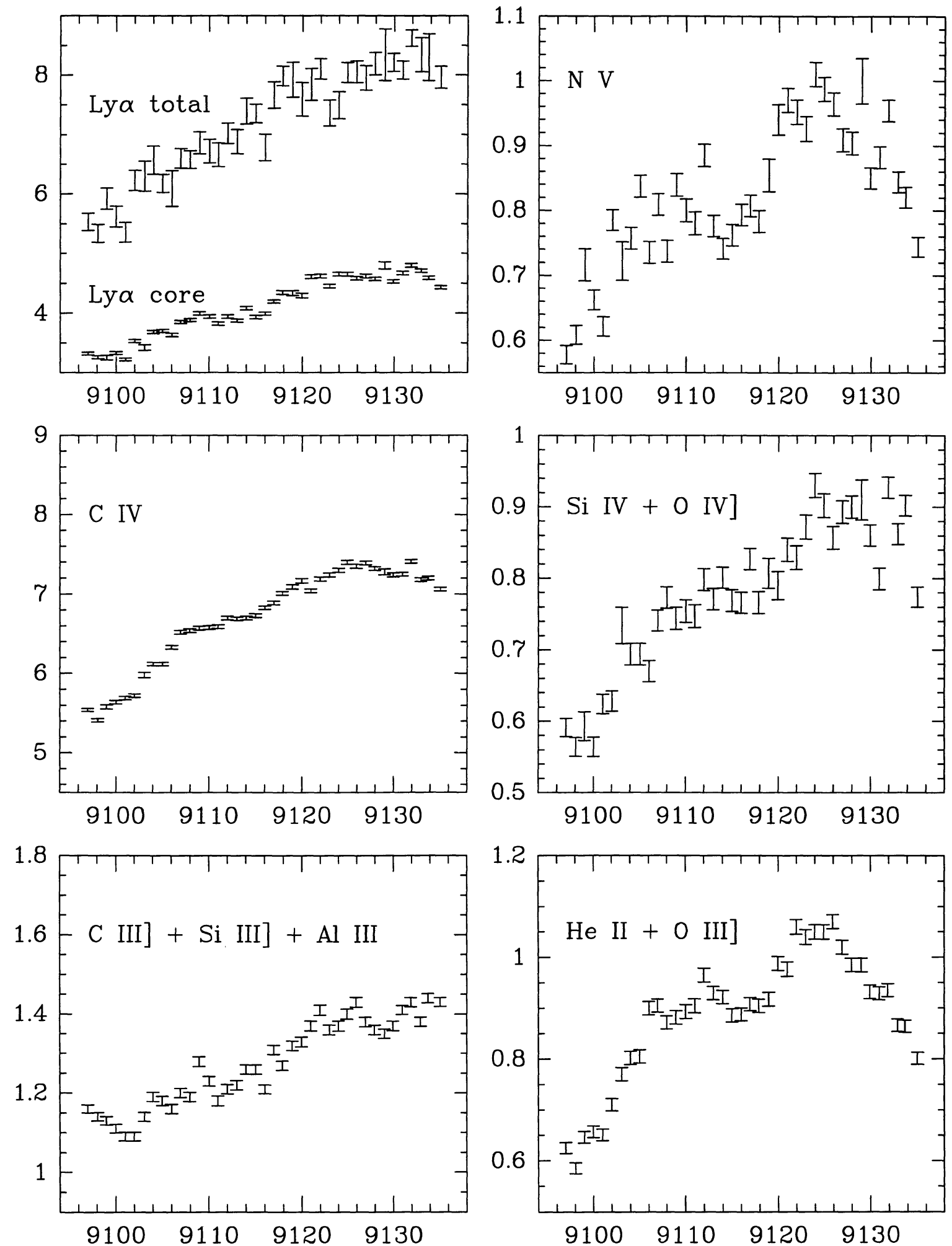

FIG. 8.-FOS direct-integration emission-light curves, as given in Tables 13 and 14. The light curve designated "Ly $\alpha$ total" was derived from the multicomponent fit. The fluxes have units of $10^{-12} \mathrm{ergs} \mathrm{s}^{-1} \mathrm{~cm}^{-2}$; the abscissa shows Julian Date minus 2,440,000. 

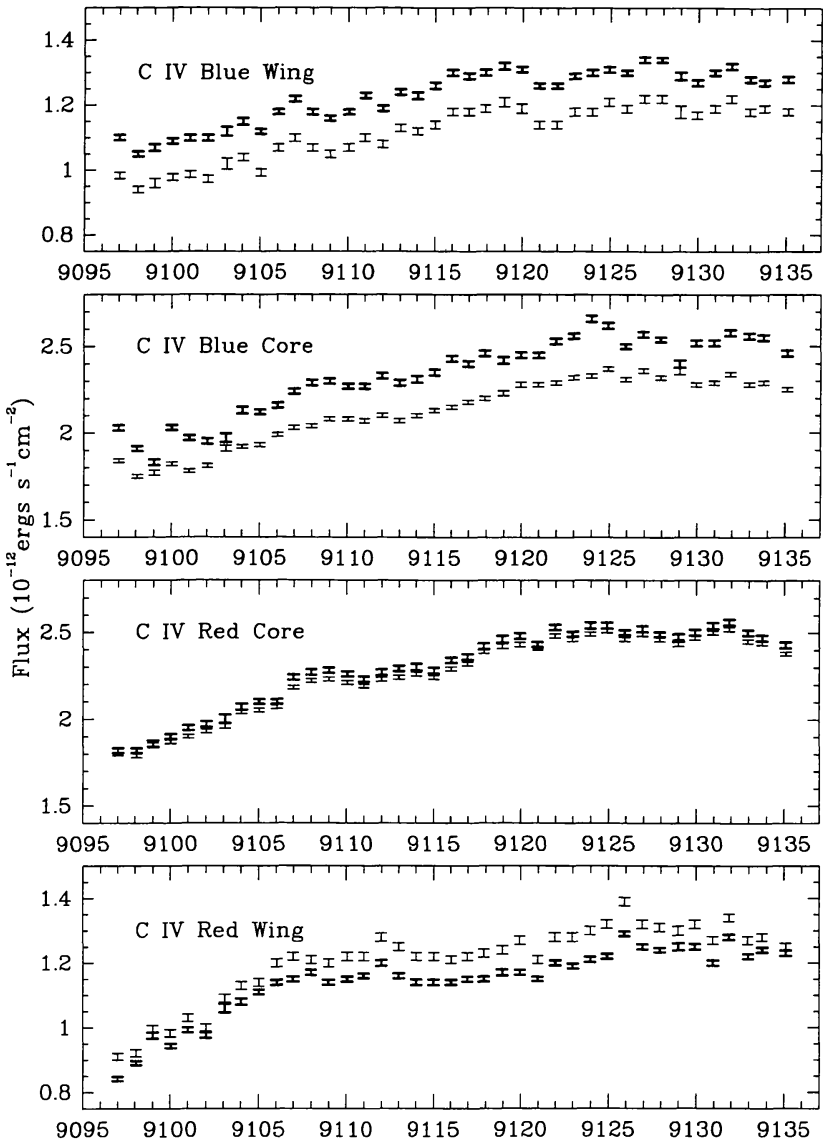

FIG. 9.-FOS emission-line light curves of the C IV profile components, as given in Table 15. The abscissa is Julian Date minus 2,440,000. The heavy symbols show the sum of the emission-line components in the multicomponent fits, and the light symbols are for the direct integrations, as described in the text.

\subsection{Absolute Calibration of the Optical Spectra}

The absolute calibration of the optical spectra is based on the flux of the [OIII] $\lambda 5007$ narrow emission line, which is assumed to be constant over the duration of the monitoring program. This is a well-founded assumption, since both the light-travel time across the narrow-line region (NLR) and the recombination time exceed 100 years. This assumption can be checked by measuring the flux in the [O III ] $\lambda 5007$ line in those spectra that were obtained under suitable conditions-specifically, when the observing conditions appeared to be of photometric quality and for which a fairly large entrance aperture was used (to reduce the importance of seeing effects). The integrated [O III] $\lambda 5007$ fluxes measured in such spectra are given in Table 18, along with the mean for the year 5 spectra. All measurements here have been transformed to the rest frame of NGC 5548, whose redshift is taken to be $z=0.0174$; the observed-frame fluxes are lower by a factor of $(1+z)^{2}$. We also give the mean values measured in previous years, as reported in Paper VII and earlier references. The year 5 [O III] $\lambda 5007$ flux is about $5 \%$ lower than the value obtained from the year 1 data, but the difference is not statistically significant. We continue to use the absolute flux given in Paper II [ F([O III] $\lambda 5007)=5.58 \times 10^{-13} \mathrm{ergs} \mathrm{s}^{-1} \mathrm{~cm}^{-2}$ ] in order to keep all of the measurements for all 5 years on the same flux scale, even though the increasing number of measurements begins to suggest that this value is too high by approximately $2 \%$.

\subsection{Optical Spectral Measurements}

Continuum (at $5100 \AA$ in the rest frame of NGC 5548) and $\mathrm{H} \beta$ emission-line measurements were made from the spectra listed in Table 3 as described in Papers II and III. The continuum and $\mathrm{H} \beta$ emission-line fluxes measured from each spectrum in which these features appear are listed in Table 19, grouped by individual homogeneous data sets. The Julian Dates associated with these measurements are those at the start of the exposure.

\subsection{Intercalibration of the Optical Spectra}

While the larger data sets in Table 19 reveal similar patterns of variability in both the continuum and the $\mathrm{H} \beta$ emission line,

TABLE 16

SWP CONTINUUM BANDS ${ }^{\mathrm{a}}$

\begin{tabular}{|c|c|c|c|}
\hline $\begin{array}{l}\text { Julian Date } \\
(2440000+)\end{array}$ & $F_{\lambda}(1350 \AA)$ & $F_{\lambda}(1460 \AA)$ & $F_{\lambda}(1790 \AA)$ \\
\hline 9060.64 & $3.66 \pm 0.176$ & $3.44 \pm 0.105$ & $2.93 \pm 0.0521$ \\
\hline 9063.04 & $2.78 \pm 0.375$ & $2.65 \pm 0.232$ & $2.37 \pm 0.110$ \\
\hline 9065.01 & $3.52 \pm 0.238$ & $3.32 \pm 0.145$ & $2.85 \pm 0.0993$ \\
\hline 9067.00 & $3.20 \pm 0.227$ & $3.08 \pm 0.140$ & $2.77 \pm 0.0687$ \\
\hline 9069.00 & $3.50 \pm 0.193$ & $3.33 \pm 0.120$ & $2.92 \pm 0.0807$ \\
\hline 9071.01 & $3.40 \pm 0.218$ & $3.20 \pm 0.136$ & $2.73 \pm 0.0804$ \\
\hline 9073.00 & $2.94 \pm 0.191$ & $2.84 \pm 0.119$ & $2.61 \pm 0.0776$ \\
\hline 9074.99 & $2.85 \pm 0.179$ & $2.73 \pm 0.111$ & $2.43 \pm 0.0657$ \\
\hline 9077.16 & $2.29 \pm 0.279$ & $2.15 \pm 0.165$ & $1.83 \pm 0.0633$ \\
\hline 9079.32 & $2.76 \pm 0.373$ & $2.61 \pm 0.226$ & $2.28 \pm 0.110$ \\
\hline 9081.41 & $2.31 \pm 0.236$ & $2.22 \pm 0.142$ & $2.02 \pm 0.0960$ \\
\hline 9082.68 & $1.44 \pm 0.131$ & $1.42 \pm 0.0830$ & $1.37 \pm 0.0500$ \\
\hline 9084.80 & $2.13 \pm 0.155$ & $2.03 \pm 0.0938$ & $1.79 \pm 0.0584$ \\
\hline 9086 & .132 & .0836 & $1.82 \pm 0.0573$ \\
\hline 9088.63 & $1.85 \pm 0.163$ & $1.81 \pm 0.100$ & $1.73 \pm 0.0729$ \\
\hline 9090.58 & $2.28 \pm 0.128$ & $2.11 \pm 0.0820$ & $1.72 \pm 0.0508$ \\
\hline 9092.93 & $2.17 \pm 0.194$ & $2.09 \pm 0.120$ & $1.90 \pm 0.0594$ \\
\hline 9094.93 & $2.18 \pm 0.184$ & $2.04 \pm 0.114$ & $1.72 \pm 0.0715$ \\
\hline 9096.93 & $1.94 \pm 0.154$ & $1.82 \pm 0.0932$ & $1.54 \pm 0.0591$ \\
\hline 9098.93 & $2.98 \pm 0.165$ & $2.77 \pm 0.0986$ & $2.29 \pm 0.0629$ \\
\hline 9100.92 & $3.17 \pm 0.170$ & $2.91 \pm 0.101$ & $2.34 \pm 0.0619$ \\
\hline 9102.93 & $3.28 \pm 0.184$ & $3.12 \pm 0.113$ & $2.72 \pm 0.0729$ \\
\hline 9104.91 & $3.69 \pm 0.223$ & $3.46 \pm 0.137$ & $2.96 \pm 0.0623$ \\
\hline 9106.91 & $3.11 \pm 0.225$ & $3.00 \pm 0.139$ & $2.73 \pm 0.0711$ \\
\hline 9108.84 & $3.81 \pm 0.160$ & $3.52 \pm 0.0952$ & $2.85 \pm 0.0603$ \\
\hline 9110.84 & $4.28 \pm 0.225$ & $3.97 \pm 0.134$ & $3.28 \pm 0.0713$ \\
\hline 9112.56 & $4.08 \pm 0.193$ & $3.74 \pm 0.112$ & $2.98 \pm 0.0682$ \\
\hline 9114.72 & $3.73 \pm 0.167$ & $3.48 \pm 0.100$ & $2.89 \pm 0.0659$ \\
\hline 9116.72 & $3.62 \pm 0.173$ & $3.39 \pm 0.103$ & $2.86 \pm 0.0678$ \\
\hline 9118.55 & $3.74 \pm 0.156$ & $3.51 \pm 0.0950$ & $2.96 \pm 0.0615$ \\
\hline 9120.57 & $4.08 \pm 0.157$ & $3.79 \pm 0.0936$ & $3.12 \pm 0.0638$ \\
\hline 9122.84 & $4.44 \pm 0.175$ & $4.17 \pm 0.105$ & $3.54 \pm 0.0675$ \\
\hline 9124.84 & $4.22 \pm 0.161$ & $3.92 \pm 0.101$ & $3.21 \pm 0.0627$ \\
\hline 9126.82 & $3.50 \pm 0.175$ & $3.32 \pm 0.107$ & $2.89 \pm 0.0682$ \\
\hline 9128.53 & $3.71 \pm 0.177$ & $3.48 \pm 0.106$ & $2.92 \pm 0.0642$ \\
\hline 9128.87 & $3.51 \pm 0.200$ & $3.37 \pm 0.123$ & $3.03 \pm 0.0640$ \\
\hline 9130.87 & $3.15 \pm 0.173$ & $3.03 \pm 0.107$ & $2.75 \pm 0.0669$ \\
\hline 9132.68 & $3.26 \pm 0.165$ & $3.05 \pm 0.101$ & $2.59 \pm 0.0629$ \\
\hline 9134.50 & $2.85 \pm 0.171$ & $2.65 \pm 0.102$ & $2.22 \pm 0.0598$ \\
\hline
\end{tabular}

${ }^{a}$ Rest-frame flux densities, in units of $10^{-14} \mathrm{ergs}$ $\mathrm{s}^{-1} \mathrm{~cm}^{-2} \AA^{-1}$. 
TABLE 17

SWP EMISSION LINES ${ }^{\mathrm{a}}$

\begin{tabular}{|c|c|c|c|c|c|c|}
\hline $\begin{array}{l}\text { Julian Date } \\
(2440000+)\end{array}$ & $\begin{array}{l}\text { Ly } \alpha \\
\text { (Fit) }\end{array}$ & $\begin{array}{c}\mathrm{Nv} \\
\text { (Direct) }\end{array}$ & $\begin{array}{c}\text { Si IV + O IV] } \\
\text { (Fit) }\end{array}$ & $\begin{array}{l}\text { C IV } \\
\text { (Fit) }\end{array}$ & $\begin{array}{l}\mathrm{He} I \mathrm{II}+\mathrm{OIII}] \\
\quad \text { (Fit) }\end{array}$ & $\underset{\text { (Fit) }}{\mathrm{C} I I I]+\mathrm{Si} I \mathrm{III}]}$ \\
\hline 9060.64 & $786 . \pm 22.0$ & $95.9 \pm 5.68$ & $80.0 \pm 9.19$ & $717 . \pm 14.8$ & $125 . \pm 4.44$ & $118 . \pm 2.17$ \\
\hline 9063.04 & $777 . \pm 30.1$ & $51.0 \pm 11.8$ & $116 . \pm 26.2$ & $702 . \pm 29.0$ & $112 . \pm 9.48$ & $142 . \pm 5.53$ \\
\hline 9065.01 & $821 . \pm 21.4$ & $75.4 \pm 7.89$ & $72.7 \pm 11.9$ & $732 . \pm 26.3$ & $113 . \pm 7.06$ & $105 . \pm 4.54$ \\
\hline 9067.00 & $765 . \pm 22.5$ & $70.6 \pm 6.27$ & $102 . \pm 14.6$ & $739 . \pm 20.4$ & $136 . \pm 5.13$ & $135 . \pm 3.55$ \\
\hline 9069.00 & $734 . \pm 17.0$ & $66.9 \pm 6.74$ & $91.9 \pm 11.5$ & $753 . \pm 25.6$ & $120 . \pm 5.97$ & $122 . \pm 4.00$ \\
\hline 9071.01 & $708 . \pm 23.0$ & $74.2 \pm 6.43$ & $112 . \pm 14.1$ & $715 . \pm 32.5$ & $193 . \pm 8.09$ & $155 . \pm 3.99$ \\
\hline 9073.00 & $729 . \pm 21.6$ & $68.6 \pm 6.08$ & $83.1 \pm 10.8$ & $761 . \pm 19.7$ & $119 . \pm 5.67$ & $125 . \pm 3.82$ \\
\hline 9074.99 & $705 . \pm 22.0$ & $66.3 \pm 6.24$ & $57.8 \pm 9.95$ & $670 . \pm 17.5$ & $91.7 \pm 4.73$ & $153 . \pm 3.38$ \\
\hline 9077.16 & $641 . \pm 20.8$ & $50.5 \pm 6.56$ & $71.3 \pm 16.6$ & $616 . \pm 24.6$ & $96.5 \pm 5.08$ & $139 . \pm 3.43$ \\
\hline 9079.32 & $635 . \pm 24.9$ & $47.1 \pm 8.83$ & $110 . \pm 24.5$ & $578 . \pm 29.5$ & $70.7 \pm 7.26$ & $117 . \pm 4.64$ \\
\hline 9081.41 & $610 . \pm 22.3$ & $50.1 \pm 6.10$ & $90.0 \pm 13.6$ & $623 . \pm 26.3$ & $96.9 \pm 6.42$ & $127 . \pm 5.71$ \\
\hline 9082.68 & $417 . \pm 14.0$ & $33.0 \pm 4.05$ & $37.6 \pm 7.38$ & $426 . \pm 13.6$ & $56.4 \pm 3.66$ & $66.5 \pm 2.62$ \\
\hline 9084.80 & $566 . \pm 19.5$ & $29.4 \pm 4.53$ & $51.7 \pm 7.67$ & $576 . \pm 21.1$ & $64.8 \pm 3.81$ & $115 . \pm 2.95$ \\
\hline 9086.59 & $503 . \pm 21.2$ & $32.5 \pm 4.24$ & $48.3 \pm 7.87$ & $506 . \pm 20.4$ & $42.5 \pm 4.15$ & $114 . \pm 3.03$ \\
\hline 9088.63 & $499 . \pm 17.7$ & $42.8 \pm 4.64$ & $60.8 \pm 7.17$ & $519 . \pm 23.8$ & $81.9 \pm 5.38$ & $144 . \pm 4.41$ \\
\hline 9090.58 & $489 . \pm 14.4$ & $32.8 \pm 4.53$ & $40.5 \pm 6.10$ & $514 . \pm 17.1$ & $62.4 \pm 3.61$ & $126 . \pm 2.68$ \\
\hline 9092.93 & $498 . \pm 15.9$ & $45.2 \pm 5.09$ & $45.2 \pm 10.8$ & $497 . \pm 16.7$ & $64.9 \pm 3.75$ & $104 . \pm 2.65$ \\
\hline 9094.93 & $416 . \pm 18.8$ & $35.4 \pm 5.73$ & $36.5 \pm 10.4$ & $391 . \pm 22.0$ & $63.7 \pm 4.56$ & $69.1 \pm 2.67$ \\
\hline 9096.93 & $418 . \pm 16.5$ & $44.6 \pm 5.06$ & $33.8 \pm 8.20$ & $415 . \pm 21.3$ & $68.6 \pm 4.63$ & $83.9 \pm 3.09$ \\
\hline 9098.93 & $529 . \pm 17.1$ & $51.5 \pm 5.68$ & $43.3 \pm 8.42$ & $567 . \pm 20.0$ & $78.8 \pm 4.56$ & $124 . \pm 3.30$ \\
\hline 9100.92 & $506 . \pm 16.3$ & $41.6 \pm 5.50$ & $48.8 \pm 8.10$ & $608 . \pm 21.4$ & $69.8 \pm 4.24$ & $110 . \pm 3.01$ \\
\hline 9102.93 & $556 . \pm 23.0$ & $68.4 \pm 7.12$ & $58.0 \pm 8.86$ & $613 . \pm 21.0$ & $94.9 \pm 4.86$ & $119 . \pm 3.51$ \\
\hline 9104.91 & $603 . \pm 17.4$ & $62.6 \pm 6.11$ & $82.4 \pm 14.8$ & $644 . \pm 27.5$ & $80.2 \pm 3.84$ & $94.8 \pm 2.60$ \\
\hline 9106.91 & $676 . \pm 17.6$ & $74.1 \pm 6.03$ & $85.6 \pm 13.2$ & $690 . \pm 22.9$ & $140 . \pm 5.13$ & $120 . \pm 3.51$ \\
\hline 9108.84 & $635 . \pm 18.4$ & $63.9 \pm 5.97$ & $68.7 \pm 8.58$ & $631 . \pm 20.0$ & $110 . \pm 4.71$ & $130 . \pm 3.04$ \\
\hline 9110.84 & $698 . \pm 22.0$ & $67.3 \pm 6.50$ & $79.7 \pm 12.7$ & $672 . \pm 30.2$ & $121 . \pm 5.08$ & $112 . \pm 3.37$ \\
\hline 9112.56 & $638 . \pm 21.2$ & $78.1 \pm 6.19$ & $61.4 \pm 9.45$ & $657 . \pm 19.4$ & $116 . \pm 5.43$ & $124 . \pm 3.49$ \\
\hline 9114.72 & $639 . \pm 17.9$ & $65.8 \pm 6.11$ & $70.7 \pm 8.67$ & $689 . \pm 17.0$ & $125 . \pm 5.48$ & $135 . \pm 3.71$ \\
\hline 9116.72 & $627 . \pm 13.0$ & $59.1 \pm 5.74$ & $74.7 \pm 9.50$ & $654 . \pm 19.0$ & $97.1 \pm 5.38$ & $134 . \pm 3.72$ \\
\hline 9118.55 & $661 . \pm 25.2$ & $64.2 \pm 6.51$ & $64.4 \pm 8.44$ & $695 . \pm 15.1$ & $97.1 \pm 5.38$ & $137 . \pm 3.12$ \\
\hline 9120.57 & $615 . \pm 15.8$ & $64.2 \pm 6.10$ & $58.8 \pm 7.90$ & $666 . \pm 14.5$ & $96.4 \pm 4.71$ & $122 . \pm 3.14$ \\
\hline 9122.84 & $790 . \pm 22.3$ & $75.5 \pm 7.12$ & $77.3 \pm 8.95$ & $723 . \pm 18.6$ & $116 . \pm 5.33$ & $109 . \pm 3.16$ \\
\hline 9124.84 & $745 . \pm 18.1$ & $85.6 \pm 6.78$ & $89.8 \pm 9.37$ & $756 . \pm 15.6$ & $134 . \pm 4.82$ & $143 . \pm 3.26$ \\
\hline 9126.82 & $692 . \pm 23.4$ & $65.2 \pm 6.38$ & $54.7 \pm 9.10$ & $670 . \pm 19.0$ & $96.0 \pm 4.94$ & $99.1 \pm 3.15$ \\
\hline 9128.53 & $729 . \pm 24.7$ & $65.2 \pm 6.14$ & $69.4 \pm 9.48$ & $737 . \pm 16.8$ & $123 . \pm 5.24$ & $132 . \pm 3.29$ \\
\hline 9128.87 & $746 . \pm 31.9$ & $71.5 \pm 6.43$ & $85.8 \pm 8.26$ & $758 . \pm 18.3$ & $98.4 \pm 4.69$ & $118 . \pm 2.96$ \\
\hline 9130.87 & $773 . \pm 21.8$ & $78.7 \pm 6.32$ & $91.0 \pm 10.3$ & $758 . \pm 18.3$ & $163 . \pm 5.43$ & $139 . \pm 3.53$ \\
\hline 9132.68 & $726 . \pm 23.4$ & $66.7 \pm 5.98$ & $113 . \pm 10.4$ & $719 . \pm 18.7$ & $124 . \pm 4.99$ & $149 . \pm 3.40$ \\
\hline 9134.50 & $704 . \pm 16.6$ & $50.8 \pm 5.44$ & $97.7 \pm 11.1$ & $719 . \pm 18.4$ & $126 . \pm 5.24$ & $147 . \pm 3.28$ \\
\hline
\end{tabular}

${ }^{a}$ Rest-frame fluxes in units of $10^{-14} \mathrm{ergs} \mathrm{s}^{-1} \mathrm{~cm}^{-2}$.

the light curves produced from the individual sets are slightly offset in flux from one another. As described in earlier papers in this series, we attribute these differences primarily to aperture effects, and as in our previous papers we apply an empirically determined correction to each of the data sets to adjust them to a common flux scale. We adopt set A, which is fairly well sampled in time and internally very homogeneous, as a standard and apply corrections to the other sets that bring measurements from the two sets that are closely spaced in time into agreement. We define a point-source correction factor $\varphi$ by the equation

$$
F(\mathrm{H} \beta)=\varphi F(\mathrm{H} \beta)_{\text {obs }},
$$

where $F(\mathrm{H} \beta)_{\text {obs }}$ is the measured $\mathrm{H} \beta$ flux from Table 19. The factor $\varphi$ accounts for the fact that different apertures result in different amounts of light loss for the PSF (which describes the surface brightness distribution of both the broad lines and the AGN continuum source) and the partially extended NLR. The correction factor is in principle a function of seeing; since no attempt is made to correct for seeing effects, this is probably our largest single source of uncertainty.

After correcting for aperture effects on the PSF to narrow line ratio, another correction needs to be applied to adjust for the different amounts of starlight admitted by different apertures. We define for this purpose an extended source correction $G$ by the equation

$$
F_{\lambda}(5100 \AA)=\varphi F_{\lambda}(5100 \AA)_{\text {obs }}-G .
$$

Determination of the constants $\varphi$ and $G$ is accomplished by comparing pairs of nearly simultaneous (to within 2 days) observations from different data sets. The formal uncertainties in $\varphi$ and $G$ reflect the uncertainties in the individual data sets, so we can determine the nominal uncertainties for each data set if we assume that the errors add in quadrature. We note that any real variability that occurs on timescales shorter than 2 days tends to be (but is not completely) suppressed by the intercalibration process that allows us to merge the different data sets. 

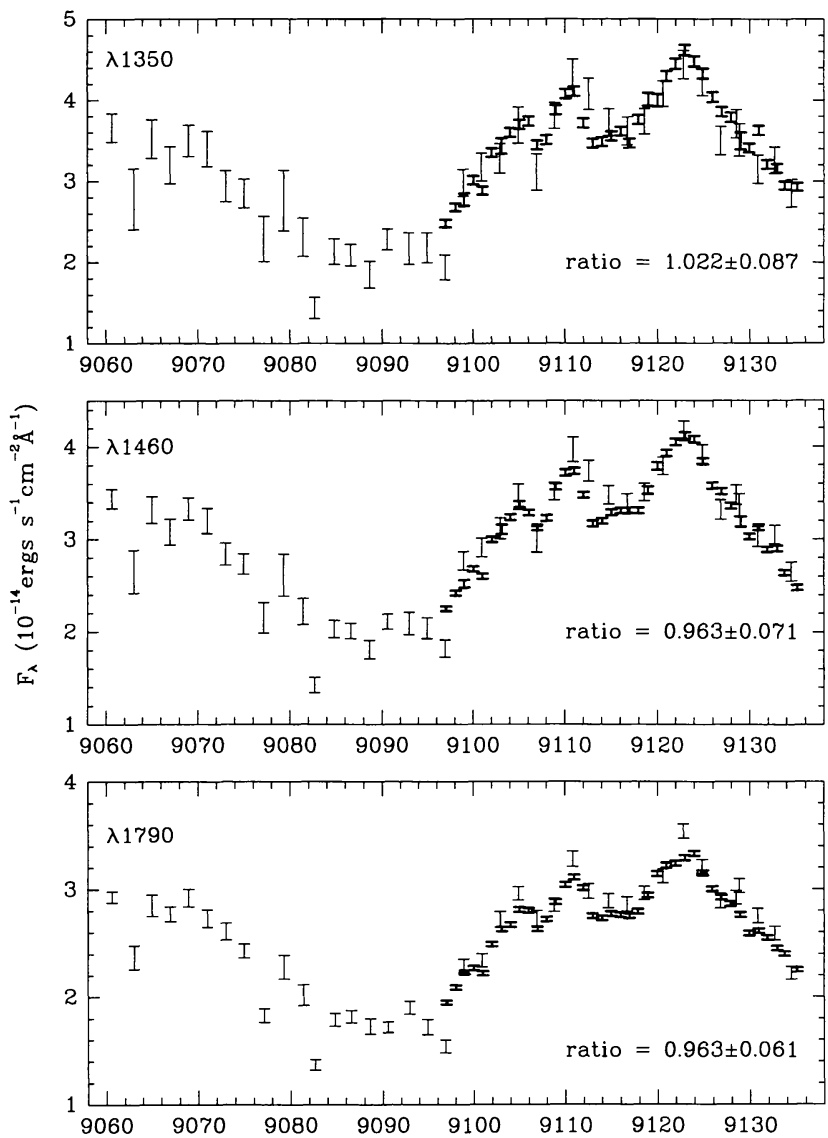

FIG. 10.-SWP continuum light curves ( light symbols), as given in Table 16, with the FOS continuum light curves overplotted (heavy symbols). The abscissa is Julian Date minus 2,440,000. In the lower right-hand corner of each panel is the median value of the distribution of the flux ratio $F(\mathrm{FOS}) / F$ (SWP) and the dispersion, for each of the 20 pairs of contemporaneous FOS and SWP spectra.

In practice, the intercalibration process is carried out by starting with the largest and most similar data sets and gradually building up a large homogenized database relative to which the smaller sets are calibrated. Fractional uncertainties of $\sigma_{\text {cont }} / F_{\lambda}(5100 \AA) \approx 0.025$ in the continuum and $\sigma_{\text {line }} /$ $F(\mathrm{H} \beta) \approx 0.025$ in the $\mathrm{H} \beta$ line are adopted for the similar, large-aperture, high-quality data sets $\mathrm{A}$ and $\mathrm{H}$, based on the differences between closely spaced observations within these sets; these values are somewhat smaller than those used previously, which we believe were too conservative. A few of the data sets are well sampled on short timescales (i.e., several pairs of observations separated by less than 2 days), and the internal fractional errors for these sets can be determined independently from the differences in the continuum and line fluxes between closely spaced pairs of observations. The fractional errors of the set $\mathrm{F}$ data are thus found to be 0.046 and 0.032 for the continuum and the $\mathrm{H} \beta$ line, respectively. Similarly, the fractional errors for set $\mathrm{W}$ are 0.025 and 0.040 , again for the continuum and line, respectively, and for set $\mathrm{Y}$ the fractional errors are 0.036 in both parameters. For set $\mathrm{B}$ the fractional errors are taken to be as given in Netzer et al. (1990), i.e., 0.040 in the continuum and 0.050 in the line. For the other data sets it is possible to estimate the mean uncertainties in the measurements by comparing them with measurements from other sets for which the uncertainties are known and by assuming that the uncertainties for each set add in quadrature. In some cases where this is not possible, the adopted fractional errors are based on the similarity of the quality of the spectra to those of other data sets for which the errors have been more reliably determined.

The intercalibration constants we use for each data set are given in Table 20, and these constants are used with equations (2) and (3) to adjust the measurements given in Table 19 to a common flux scale, which corresponds to measurements through the $5 " .0 \times 7 " .5$ spectrograph entrance aperture used in set $\mathrm{A}$. The adjusted values of the continuum flux, $F_{\lambda}(5100 \AA)$, and the $\mathrm{H} \beta$ line flux, $F(\mathrm{H} \beta)$, are given in Table 21. A final check of our uncertainty estimates can be performed by examining the ratios of all 223 pairs of observations in Table 21 that are separated by 2 days or less. The dispersion about the mean (unity), divided by $\sqrt{2}$, provides an estimate of the typical uncertainty in a single measurement. For the continuum, we find that the mean fractional error in a given measurement is 0.030 , whereas the average fractional uncertainty quoted for these points is 0.036 . Similarly, for the $\mathrm{H} \beta$ line, the mean fractional error we compute from the 223 pairs is 0.029 , slightly lower than the average value of 0.034 quoted for these same points. Thus, analysis indicates that, on average, our quoted errors are probably quite good, and if anything are slightly conservative.

\subsection{Intercalibration of the Optical Photometry}

The broadband optical fluxes given in Tables 4-10 show qualitative agreement with the behavior of the $5100 \AA$ continuum variability as determined from the spectra, but again with some systematic differences between what are taken to be internally homogeneous data sets. Most of the differences among the various data can be accounted for by the differences in the amount of starlight that enters apertures of different sizes. However, we find that after correcting for the differences in starlight contamination (as described below), small systematic

TABLE 18

absolute Calibration Check for Optical Spectra

\begin{tabular}{|c|c|}
\hline $\begin{array}{c}F([\mathrm{O} \mathrm{III}] \lambda 5007) \\
\left(10^{-13} \mathrm{ergs} \mathrm{cm}^{-2} \mathrm{~s}^{-1}\right) \\
(1)\end{array}$ & $\begin{array}{l}\text { FILE NAME } \\
\text { (2) }\end{array}$ \\
\hline $5.62 \ldots \ldots \ldots \ldots \ldots \ldots \ldots$ & n59091h \\
\hline 5.12 & $\mathrm{n} 59163 \mathrm{a}$ \\
\hline 5.40 & $\mathrm{n} 59166 \mathrm{~h}$ \\
\hline 5.41 & n59176a \\
\hline 5.31 & $\mathrm{n} 59182 \mathrm{~h}$ \\
\hline 5.05 & n59183a \\
\hline $5.22 \ldots \ldots \ldots \ldots \ldots \ldots \ldots \ldots \ldots \ldots$ & $\mathrm{n} 59212 \mathrm{~h}$ \\
\hline 5.30 & $\mathrm{n} 59240 \mathrm{~h}$ \\
\hline 5.19 & n59243h \\
\hline $5.29 \pm 0.17 \ldots \ldots \ldots \ldots \ldots$ & Mean value from year 5 \\
\hline $5.45 \pm 0.23 \ldots \ldots \ldots \ldots \ldots$ & Mean value from years 3-4 \\
\hline $5.48 \pm 0.24 \ldots \ldots \ldots \ldots \ldots \ldots$ & Mean value from year 2 \\
\hline $5.58 \pm 0.27 \ldots \ldots \ldots \ldots \ldots \ldots$ & $\begin{array}{l}\text { Mean value from year } 1 \\
\text { (adopted absolute flux) }\end{array}$ \\
\hline
\end{tabular}



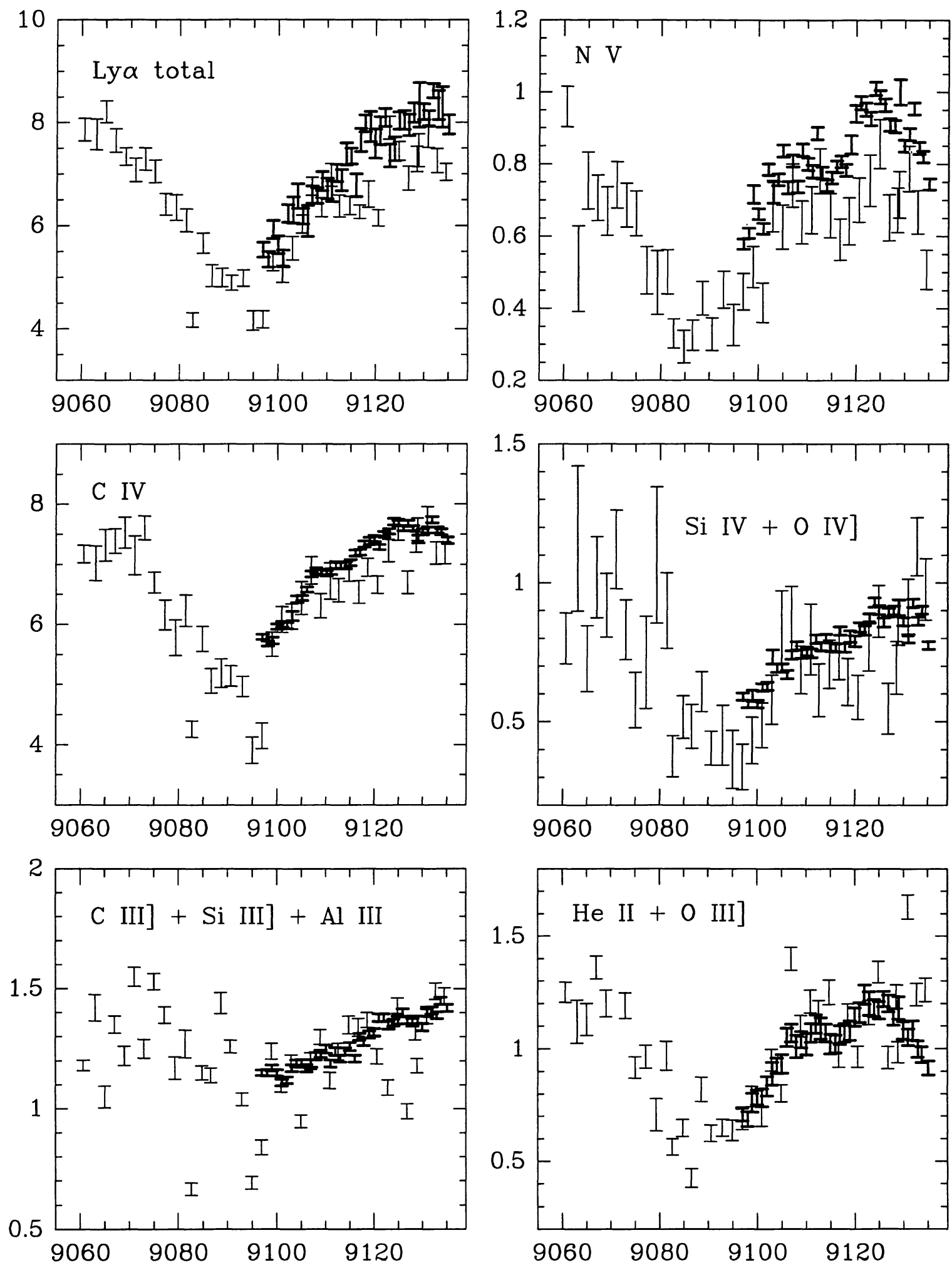

FIG. 11.-SWP emission-line light curves (light symbols), as given in Table 17, with the corresponding FOS emission light curves overplotted (heavy symbols); the fluxes shown are based on the sum of the fitted components for each line except $\mathrm{N} v$, for which the direct-integration results are shown. The fluxes have units of $10^{-12} \mathrm{ergs} \mathrm{s}^{-1} \mathrm{~cm}^{-2}$; the abscissa is Julian Date minus 2,440,000. 
TABLE 19

MEASUREMENTS OF SPECTRA

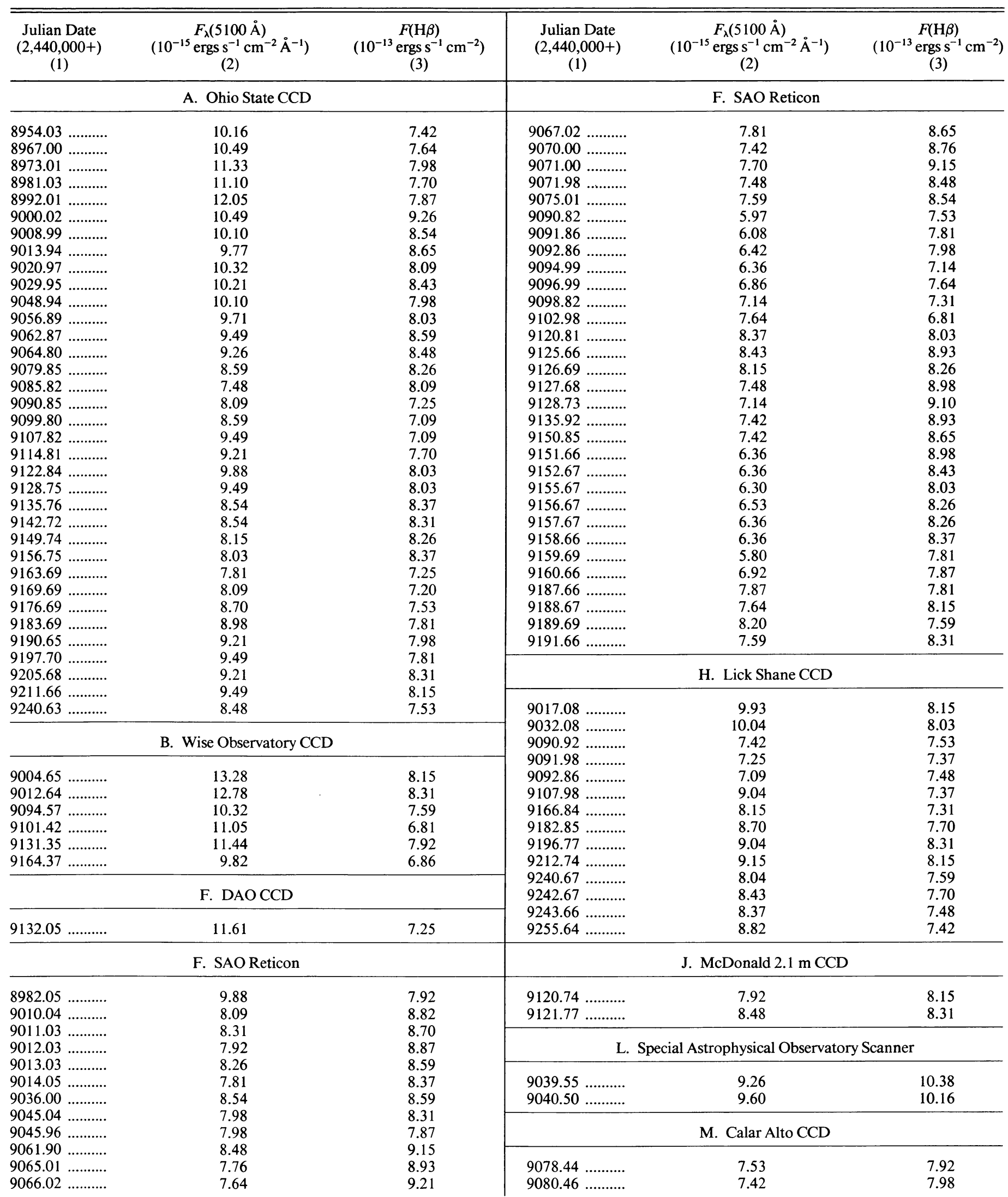


TABLE 19-Continued

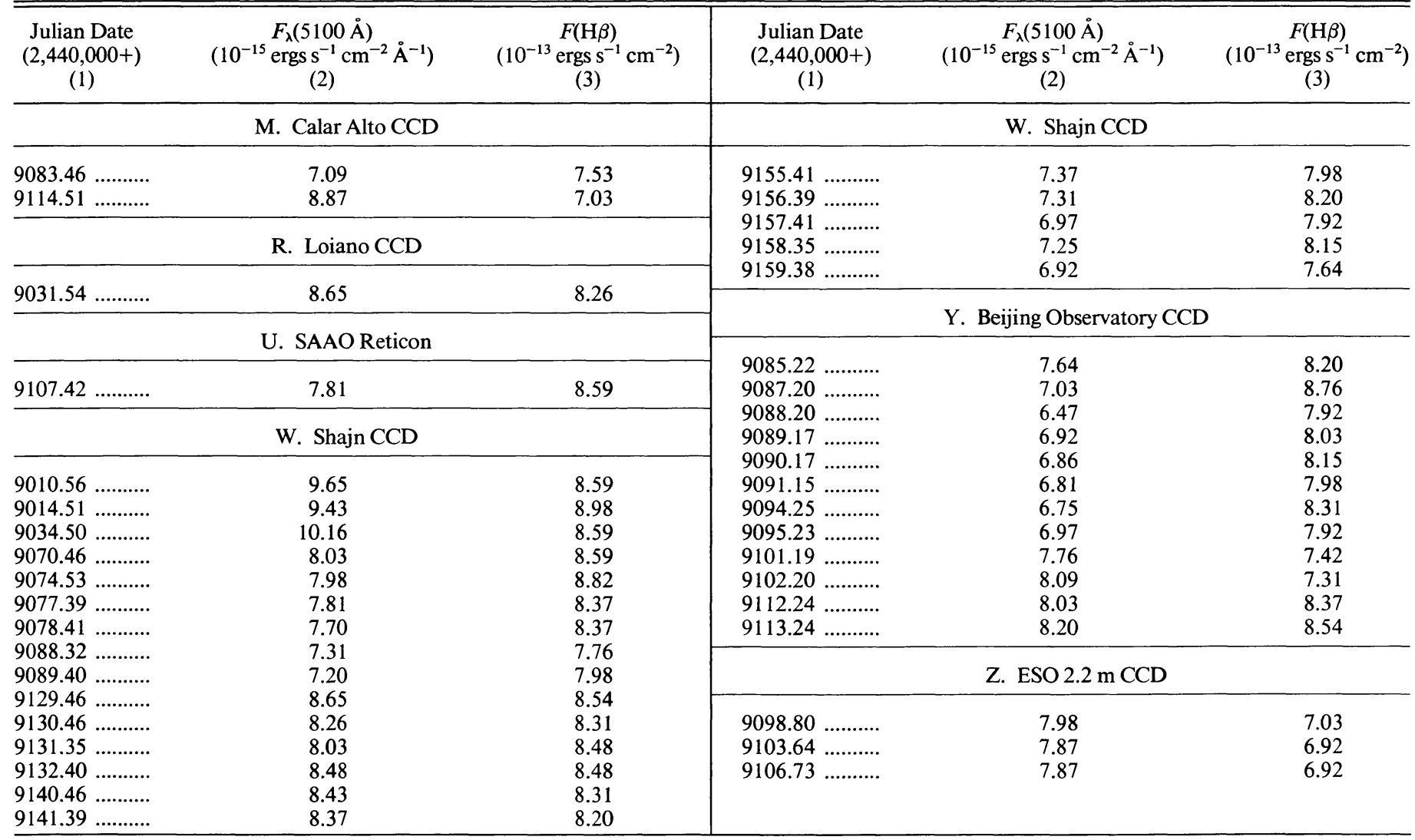

offsets remain. We ascribe these differences to somewhat different wavelength sensitivities of the different filter and detector systems that were employed at the various observatories, and we therefore compute a small empirical correction for each data set.

In order to account for the different amounts of starlight affecting the various data, we use the carefully constructed model of the starlight surface brightness distribution of Romanishin et al. (1994). This model gives as the stellar contri-

TABLE 20

FLUX SCALE FACTORS FOR OPTICAL SPECTRA

\begin{tabular}{lcc}
\hline \hline & & \\
Data & Point-Source \\
Set & $\begin{array}{c}\text { Scale Factor } \\
\varphi\end{array}$ & $\begin{array}{c}\text { Extended Source } \\
\text { Correction } G \\
\left(10^{-15} \text { ergs s}^{-1} \mathrm{~cm}^{-2} \AA^{-1}\right)\end{array}$ \\
$(1)$ & $(2)$ & $(3)$ \\
\hline $\mathrm{A}$ & 1.000 & 0.000 \\
$\mathrm{~B}$ & $1.016 \pm 0.041$ & $2.691 \pm 0.433$ \\
$\mathrm{E}$ & $1.139 \pm 0.020$ & $4.264 \pm 0.193$ \\
$\mathrm{~F}$ & $0.967 \pm 0.046$ & $-1.849 \pm 0.384$ \\
$\mathrm{H}$ & $0.981 \pm 0.025$ & $-0.711 \pm 0.231$ \\
$\mathrm{~J}$ & $0.954 \pm 0.015$ & $-2.025 \pm 0.335$ \\
$\mathrm{~L}$ & 0.803 & -2.687 \\
$\mathrm{M}$ & $1.058 \pm 0.040$ & $0.448 \pm 0.494$ \\
$\mathrm{R}$ & $0.987 \pm 0.046$ & $-1.847 \pm 0.250$ \\
$\mathrm{U}$ & $0.827 \pm 0.014$ & $-2.893 \pm 0.320$ \\
$\mathrm{~W}$ & $0.988 \pm 0.048$ & $-0.833 \pm 0.293$ \\
$\mathrm{Y}$ & $0.931 \pm 0.037$ & $-1.507 \pm 0.336$ \\
$\mathrm{Z}$ & $1.011 \pm 0.034$ & $-1.030 \pm 0.482$ \\
\hline
\end{tabular}

bution through the nominal spectroscopic aperture $(5$ ".0 $\times$ 7".5) $V_{\text {gal }}=14.99 \mathrm{mag}$, or $F_{\lambda}(5100 \AA)=3.4 \times 10^{-15} \mathrm{ergs} \mathrm{s}^{-1}$ $\mathrm{cm}^{-2} \mathrm{~A}^{-1}$. The starlight contribution through the nominal broadband aperture of radius $R=8.0$ is $V_{\text {gal }}=14.09$ mag.

We compute a zero-point photometric correction for each of the data sets in Tables 4-10 by comparing these measurements with the spectroscopic continuum measurements given in Table 21. We first convert the fluxes in Table 21 to nuclear (i.e., starlight-free) magnitudes $V_{\text {nuc }}$ by using the empirical relation between $F_{\lambda}(5100 \AA)$ and nuclear flux given by Romanishin et al. (1994) and then converting to a $V$-magnitude scale (Johnson 1966). For each of the photometric data sets, we then compute a comparison light curve by adding to the $V_{\text {nuc }}$ light curve an appropriate constant starlight contribution for the aperture employed. We can now compare directly the photometrically measured values with the spectroscopically derived comparison values to obtain a systematic correction to the photometric values. We define a zero-point correction $\Delta m$ by

$$
V_{\text {corr }}=V_{\text {obs }}+\Delta m
$$

where the $V_{\text {obs }}$ are the observed values given in Tables 4-10. The values of $\Delta m$ are empirically derived by comparing the observed values $V_{\text {obs }}$ with spectroscopically derived values that are separated in time by no more than 2 days. The appropriate values of $\Delta m$ for each photometric data set are given in Table 22. In general, the photometric zero-point adjustments are found to be very small. As in the case of the spectroscopic mea- 


\begin{tabular}{|c|c|c|c|c|c|}
\hline 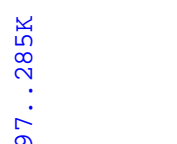 & & $\begin{array}{r}\text { TAB } \\
\text { PTICAl ContinuUm }\end{array}$ & $\begin{array}{l}\mathrm{E} 21 \\
\text { AND } \mathrm{H} \beta \mathrm{LIG}\end{array}$ & HT Curves & \\
\hline $\begin{array}{l}\text { DUulian Date } \\
\text { is }\end{array}$ & $\begin{array}{c}F_{\lambda}(5100 \AA) \\
\left(10^{-15} \mathrm{ergs} \mathrm{s}^{-1} \mathrm{~cm}^{-2} \AA^{-1}\right) \\
(2)\end{array}$ & $\begin{array}{c}F(\mathrm{H} \beta) \\
\left(10^{-13} \mathrm{ergs} \mathrm{s}^{-1} \mathrm{~cm}^{-2}\right) \\
(3)\end{array}$ & $\begin{array}{c}\text { Julian Date } \\
(2440000+) \\
(1)\end{array}$ & $\begin{array}{c}F_{\lambda}(5100 \AA) \\
\left(10^{-15} \mathrm{ergs} \mathrm{s}^{-1} \mathrm{~cm}^{-2} \AA^{-1}\right) \\
(2)\end{array}$ & $\begin{array}{c}F(\mathrm{H} \beta) \\
\left(10^{-13} \mathrm{ergs} \mathrm{s}^{-1} \mathrm{~cm}^{-2}\right) \\
(3)\end{array}$ \\
\hline भू 8954.03 & $10.16 \pm 0.25$ & $7.42 \pm 0.19$ & 9099.80 & $8.59 \pm 0.22$ & $7.09 \pm 0.18$ \\
\hline I斤 8967.00 & $10.49 \pm 0.26$ & $7.64 \pm 0.19$ & 9101.19 & $8.73 \pm 0.31$ & $6.91 \pm 0.25$ \\
\hline レ 8973.01 & $11.33 \pm 0.28$ & $7.98 \pm 0.20$ & 9101.42 & $8.53 \pm 0.34$ & $6.92 \pm 0.35$ \\
\hline 8981.03 & $11.10 \pm 0.28$ & $7.70 \pm 0.19$ & 9102.20 & $9.04 \pm 0.32$ & $6.81 \pm 0.25$ \\
\hline 8982.05 & $11.43 \pm 0.53$ & $7.69 \pm 0.26$ & 9102.98 & $9.26 \pm 0.43$ & $6.60 \pm 0.22$ \\
\hline 8992.01 & $12.05 \pm 0.30$ & $7.87 \pm 0.20$ & 9103.64 & $8.98 \pm 0.45$ & $6.99 \pm 0.25$ \\
\hline 9000.02 & $10.49 \pm 0.26$ & $9.26 \pm 0.23$ & 9106.73 & $8.98 \pm 0.45$ & $6.99 \pm 0.25$ \\
\hline 9004.65 & $10.80 \pm 0.43$ & $8.28 \pm 0.41$ & 9107.42 & $9.35 \pm 0.47$ & $7.11 \pm 0.28$ \\
\hline 9008.99 & $10.10 \pm 0.25$ & $8.54 \pm 0.21$ & 9107.82 & $9.49 \pm 0.24$ & $7.09 \pm 0.18$ \\
\hline 9010.04 & $9.69 \pm 0.45$ & $8.55 \pm 0.29$ & 9107.98 & $9.58 \pm 0.24$ & $7.23 \pm 0.18$ \\
\hline 9010.56 & $10.37 \pm 0.26$ & $8.49 \pm 0.34$ & 9112.24 & $8.99 \pm 0.32$ & $7.79 \pm 0.28$ \\
\hline 9011.03 & $9.91 \pm 0.46$ & $8.44 \pm 0.29$ & 9113.24 & $9.14 \pm 0.33$ & $7.95 \pm 0.29$ \\
\hline 9012.03 & $9.53 \pm 0.44$ & $8.61 \pm 0.29$ & 9114.51 & $9.84 \pm 0.49$ & $7.44 \pm 0.26$ \\
\hline 9012.64 & $10.29 \pm 0.41$ & $8.45 \pm 0.42$ & 9114.81 & $9.21 \pm 0.23$ & $7.70 \pm 0.19$ \\
\hline 9013.03 & $9.85 \pm 0.45$ & $8.34 \pm 0.28$ & 9120.74 & $9.58 \pm 0.48$ & $7.77 \pm 0.27$ \\
\hline 9013.94 & $9.77 \pm 0.24$ & $8.65 \pm 0.22$ & 9120.81 & $9.96 \pm 0.46$ & $7.79 \pm 0.26$ \\
\hline 9014.05 & $9.42 \pm 0.43$ & $8.12 \pm 0.28$ & 9121.77 & $10.12 \pm 0.51$ & $7.93 \pm 0.28$ \\
\hline 9014.51 & $10.15 \pm 0.25$ & $8.88 \pm 0.35$ & 9122.84 & $9.88 \pm 0.25$ & $8.03 \pm 0.20$ \\
\hline 9017.08 & $10.45 \pm 0.26$ & $7.99 \pm 0.20$ & 9125.66 & $10.02 \pm 0.46$ & $8.66 \pm 0.29$ \\
\hline 9020.97 & $10.32 \pm 0.26$ & $8.09 \pm 0.20$ & 9126.69 & $9.75 \pm 0.45$ & $8.01 \pm 0.27$ \\
\hline 9029.95 & $10.21 \pm 0.25$ & $8.43 \pm 0.21$ & 9127.68 & $9.10 \pm 0.42$ & $8.71 \pm 0.30$ \\
\hline 9031.54 & $10.38 \pm 0.52$ & $8.15 \pm 0.33$ & 9128.73 & $8.77 \pm 0.40$ & $8.82 \pm 0.30$ \\
\hline 9032.08 & $10.56 \pm 0.26$ & $7.88 \pm 0.20$ & 9128.75 & $9.49 \pm 0.24$ & $8.03 \pm 0.20$ \\
\hline 9034.50 & $10.87 \pm 0.27$ & $8.49 \pm 0.34$ & 9129.46 & $9.38 \pm 0.23$ & $8.44 \pm 0.34$ \\
\hline 9036.00 & $10.13 \pm 0.47$ & $8.34 \pm 0.28$ & 9130.46 & $8.99 \pm 0.22$ & $8.21 \pm 0.33$ \\
\hline 9039.55 & $10.12 \pm 0.61$ & $8.33 \pm 0.54$ & 9131.35 & $8.82 \pm 0.19$ & $8.25 \pm 0.26$ \\
\hline 9040.50 & $10.39 \pm 0.62$ & $8.15 \pm 0.53$ & 9132.05 & $8.95 \pm 0.54$ & $8.26 \pm 0.62$ \\
\hline 9045.04 & $9.59 \pm 0.44$ & $8.06 \pm 0.27$ & 9132.40 & $9.21 \pm 0.23$ & $8.38 \pm 0.34$ \\
\hline 9045.96 & $9.59 \pm 0.44$ & $7.63 \pm 0.26$ & 9135.76 & $8.54 \pm 0.21$ & $8.37 \pm 0.21$ \\
\hline 9048.94 & $10.10 \pm 0.25$ & $7.98 \pm 0.20$ & 9135.92 & $9.04 \pm 0.42$ & $8.66 \pm 0.29$ \\
\hline 9056.89 & $9.71 \pm 0.24$ & $8.03 \pm 0.20$ & 9140.46 & $9.16 \pm 0.23$ & $8.21 \pm 0.33$ \\
\hline 9061.90 & $10.07 \pm 0.46$ & $8.88 \pm 0.30$ & 9141.39 & $9.10 \pm 0.23$ & $8.10 \pm 0.32$ \\
\hline 9062.87 & $9.49 \pm 0.24$ & $8.59 \pm 0.22$ & 9142.72 & $8.54 \pm 0.21$ & $8.31 \pm 0.21$ \\
\hline 9064.80 & $9.26 \pm 0.23$ & $8.48 \pm 0.21$ & 9149.74 & $8.15 \pm 0.20$ & $8.26 \pm 0.21$ \\
\hline 9065.01 & $9.37 \pm 0.43$ & $8.66 \pm 0.29$ & 9150.85 & $9.04 \pm 0.42$ & $8.39 \pm 0.28$ \\
\hline 9066.02 & $9.26 \pm 0.43$ & $8.93 \pm 0.30$ & 9151.66 & $8.02 \pm 0.37$ & $8.71 \pm 0.30$ \\
\hline 9067.02 & $9.42 \pm 0.43$ & $8.39 \pm 0.28$ & 9152.67 & $8.02 \pm 0.37$ & $8.17 \pm 0.28$ \\
\hline 9070.00 & $9.04 \pm 0.42$ & $8.50 \pm 0.29$ & 9155.41 & $8.11 \pm 0.20$ & $7.88 \pm 0.31$ \\
\hline 9070.46 & $8.77 \pm 0.22$ & $8.49 \pm 0.34$ & 9155.67 & $7.96 \pm 0.37$ & $7.79 \pm 0.26$ \\
\hline 9071.00 & $9.31 \pm 0.43$ & $8.88 \pm 0.30$ & 9156.39 & $8.06 \pm 0.20$ & $8.10 \pm 0.32$ \\
\hline 9071.98 & $9.10 \pm 0.42$ & $8.23 \pm 0.28$ & 9156.67 & $8.18 \pm 0.38$ & $8.01 \pm 0.27$ \\
\hline 9074.53 & $8.72 \pm 0.22$ & $8.71 \pm 0.35$ & 9156.75 & $8.03 \pm 0.20$ & $8.37 \pm 0.21$ \\
\hline 9075.01 & $9.21 \pm 0.42$ & $8.28 \pm 0.28$ & 9157.41 & $7.72 \pm 0.19$ & $7.83 \pm 0.31$ \\
\hline 9077.39 & $8.55 \pm 0.21$ & $8.27 \pm 0.33$ & 9157.67 & $8.02 \pm 0.37$ & $8.01 \pm 0.27$ \\
\hline 9078.41 & $8.44 \pm 0.21$ & $8.27 \pm 0.33$ & 9158.35 & $8.00 \pm 0.20$ & $8.05 \pm 0.32$ \\
\hline 9078.44 & $8.42 \pm 0.42$ & $8.38 \pm 0.29$ & 9158.66 & $8.02 \pm 0.37$ & $8: 12 \pm 0.28$ \\
\hline 9079.85 & $8.59 \pm 0.22$ & $8.26 \pm 0.21$ & 9159.38 & $7.67 \pm 0.19$ & $7.55 \pm 0.30$ \\
\hline 9080.46 & $8.30 \pm 0.41$ & $8.44 \pm 0.29$ & 9159.69 & $7.47 \pm 0.34$ & $7.58 \pm 0.26$ \\
\hline 9083.46 & $7.95 \pm 0.40$ & $7.97 \pm 0.28$ & 9160.66 & $8.56 \pm 0.39$ & $7.63 \pm 0.26$ \\
\hline 9085.22 & $8.62 \pm 0.31$ & $7.64 \pm 0.28$ & 9163.69 & $7.81 \pm 0.19$ & $7.25 \pm 0.18$ \\
\hline 9085.82 & $7.48 \pm 0.19$ & $8.09 \pm 0.20$ & 9164.37 & $7.29 \pm 0.29$ & $6.97 \pm 0.35$ \\
\hline 9087.20 & $8.05 \pm 0.29$ & $8.16 \pm 0.29$ & 9166.84 & $8.70 \pm 0.22$ & $7.17 \pm 0.18$ \\
\hline 9088.20 & $7.53 \pm 0.27$ & $7.38 \pm 0.27$ & 9169.69 & $8.09 \pm 0.20$ & $7.20 \pm 0.18$ \\
\hline 9088.32 & $8.06 \pm 0.20$ & $7.66 \pm 0.31$ & 9176.69 & $8.70 \pm 0.22$ & $7.53 \pm 0.19$ \\
\hline 9089.17 & $7.95 \pm 0.29$ & $7.48 \pm 0.27$ & 9182.85 & $9.25 \pm 0.23$ & $7.55 \pm 0.19$ \\
\hline 9089.40 & $7.95 \pm 0.20$ & $7.88 \pm 0.31$ & 9183.69 & $8.98 \pm 0.22$ & $7.81 \pm 0.19$ \\
\hline 9090.17 & $7.90 \pm 0.28$ & $7.59 \pm 0.27$ & 9187.66 & $9.48 \pm 0.44$ & $7.58 \pm 0.26$ \\
\hline 9090.82 & $7.64 \pm 0.35$ & $7.31 \pm 0.25$ & 9188.67 & $9.26 \pm 0.43$ & $7.90 \pm 0.27$ \\
\hline 9090.85 & $8.09 \pm 0.20$ & $7.25 \pm 0.18$ & 9189.69 & $9.80 \pm 0.45$ & $7.36 \pm 0.25$ \\
\hline 9090.92 & $7.99 \pm 0.20$ & $7.39 \pm 0.19$ & 9190.65 & $9.21 \pm 0.23$ & $7.98 \pm 0.20$ \\
\hline 9091.15 & $7.84 \pm 0.28$ & $7.43 \pm 0.27$ & 9191.66 & $9.21 \pm 0.42$ & $8.06 \pm 0.27$ \\
\hline 9091.86 & $7.74 \pm 0.36$ & $7.58 \pm 0.26$ & 9196.77 & $9.58 \pm 0.24$ & $8.16 \pm 0.20$ \\
\hline 9091.98 & $7.83 \pm 0.20$ & $7.23 \pm 0.18$ & 9197.70 & $9.49 \pm 0.24$ & $7.81 \pm 0.19$ \\
\hline 9092.86 & $7.75 \pm 0.17$ & $7.46 \pm 0.15$ & 9205.68 & $9.21 \pm 0.23$ & $8.31 \pm 0.21$ \\
\hline 9094.25 & $7.79 \pm 0.28$ & $7.74 \pm 0.28$ & 9211.66 & $9.49 \pm 0.24$ & $8.15 \pm 0.20$ \\
\hline 9094.57 & $7.80 \pm 0.31$ & $7.71 \pm 0.39$ & 9212.74 & $9.69 \pm 0.24$ & $7.99 \pm 0.20$ \\
\hline 9094.99 & $8.02 \pm 0.37$ & $6.93 \pm 0.24$ & 9240.63 & $8.48 \pm 0.21$ & $7.53 \pm 0.19$ \\
\hline 9095.23 & $8.00 \pm 0.29$ & $7.38 \pm 0.27$ & 9240.67 & $8.59 \pm 0.22$ & $7.45 \pm 0.19$ \\
\hline 9096.99 & $8.50 \pm 0.39$ & $7.42 \pm 0.25$ & 9242.67 & $8.98 \pm 0.22$ & $7.55 \pm 0.19$ \\
\hline 9098.80 & $9.10 \pm 0.46$ & $7.11 \pm 0.25$ & 9243.66 & $8.92 \pm 0.22$ & $7.34 \pm 0.18$ \\
\hline 9098.82 & $8.77 \pm 0.40$ & $7.09 \pm 0.24$ & 9255.64 & $9.36 \pm 0.23$ & $7.28 \pm 0.18$ \\
\hline
\end{tabular}


TABLE 22

PHOTOMETRIC ZERO-POINT ADJUSTMENTS

\begin{tabular}{|c|c|}
\hline $\begin{array}{l}\text { Data Set } \\
\text { (1) }\end{array}$ & $\begin{array}{c}\Delta m \\
(\mathrm{mag}) \\
(2)\end{array}$ \\
\hline Lowell CCD (Table 4) & $-0.025 \pm 0.018$ \\
\hline MSU CCD (Table 5) .. & $0.019 \pm 0.020$ \\
\hline San Pedro Mártir CCD (Table 6) ........... & $-0.079 \pm 0.019$ \\
\hline 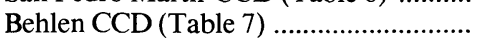 & $0.030 \pm 0.040$ \\
\hline 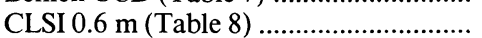 & $-0.045 \pm 0.037$ \\
\hline CAO 1.25 m (Table 9) ............ & $0.017 \pm 0.039$ \\
\hline CBA 0.3 m CCD (Table 10) .................... & $-0.180 \pm 0.027$ \\
\hline
\end{tabular}

surements, this intercalibration process also allows us to make more accurate estimates of the mean uncertainties in each data set.

After adjustment of the photometric zero point for each set of data, the appropriate starlight contribution is subtracted again to yield values of $V_{\text {nuc }}$ which are based on the photometric data. These values are given in Table 23 for each of the photometric measurements. It is then straightforward to convert these to values of $F_{\lambda}(5100 \AA)$, and these values are also given in Table 23.

The combined $5100 \AA$ continuum and $\mathrm{H} \beta$ emission-line light curves from Tables 21 and 23 are shown in Figure 12. Marked on Figure 12 are the time spans of the IUE and HST campaigns. As noted earlier, the beginning of the HST campaign was delayed by 2 weeks on account of a spacecraft safemode condition that was triggered by a solar-array problem. An unfortunate consequence of this delay is that the optical observations, which were arranged on the basis of the original $H S T$ schedule, were relatively sparse near the end of the $H S T$ campaign, and the $H S T$ observations did not overlap with the time period of the best temporal coverage of the ground-based campaign. By circumstance, the $H S T$ observations also just missed the large decline and turnaround in the continuum that occurred around JD 2,449,090.

\section{VARIABILITY ANALYSIS}

\subsection{Characteristics of the Ultraviolet Database}

The parameters we use to characterize the variability are $F_{\text {var }}$, the ratio of the rms fluctuation to the mean flux, corrected for the effect of measurement errors (see Paper I), and $R_{\max }$, the ratio of maximum to minimum flux. The mean flux and these two variability parameters are listed in columns (5)-(7) of Table 24 for many of the continuum and emission-line measurements. The parameters derived for the " $H S T$ only" features refer to the direct-integration measurements only (with the exception of the total Ly $\alpha$ flux ). When combining the IUE and HST measurements into a light curve for time-series analysis, the FOS measurements were simply appended to the SWP measurements that were obtained before the HST campaign. The combined light-curve data thus consist of those data that are featured in Figures 10 and 11, excluding the IUE data obtained after the beginning of the $H S T$ campaign. The inclusion of the later $I U E$ data would merely add non-Poissonian noise to the light curve without improving the temporal sampling. The measurements were not scaled or otherwise adjusted before being combined in this fashion. We designate these as the "combined ultraviolet" data set. For the purposes of this analysis, four additional SWP spectra also are excluded: SWP 47290 (JD 2,449,063), SWP 47387 (JD 2,449,077), SWP 47422 (JD 2,449,082), and SWP 47496 (JD 2,449,095). All of these either are underexposed or are suspected of having been misplaced in the aperture. These types of problems could induce errors between the emission lines and continuum which are correlated in time, thus biasing the derived cross-correlation lags toward zero time delay $(\S 6.3)$. Since the lags that we expect to measure from these light curves are small, the most

TABLE 23

\begin{tabular}{|c|c|c|}
\hline $\begin{array}{l}\text { Julian Date } \\
(2440000+) \\
(1)\end{array}$ & $\begin{array}{c}V_{n u c} \\
\text { (magnitudes) } \\
(2)\end{array}$ & $\begin{array}{c}F_{\lambda}(5100 \AA) \\
\left(10^{-15} \mathrm{ergs} \mathrm{s}^{-1} \mathrm{~cm}^{-2} \AA^{-1}\right) \\
(3)\end{array}$ \\
\hline 8991.62 & $14.17 \pm 0.06$ & $10.86 \pm 0.60$ \\
\hline 9047.60 & $14.42 \pm 0.06$ & $9.23 \pm 0.51$ \\
\hline 9053.78 & $14.31 \pm 0.06$ & $9.90 \pm 0.55$ \\
\hline 9059.40 & $14.33 \pm 0.06$ & $9.77 \pm 0.54$ \\
\hline 9061.57 & $14.36 \pm 0.06$ & $9.59 \pm 0.53$ \\
\hline 9062.56 & $14.36 \pm 0.06$ & $9.59 \pm 0.53$ \\
\hline 9063.54 & $14.40 \pm 0.06$ & $9.34 \pm 0.52$ \\
\hline 9063.82 & $14.38 \pm 0.02$ & $9.46 \pm 0.17$ \\
\hline 9069.45 & $14.47 \pm 0.06$ & $8.94 \pm 0.49$ \\
\hline 9073.95 & $14.52 \pm 0.02$ & $8.67 \pm 0.16$ \\
\hline 9078.90 & $14.57 \pm 0.02$ & $8.41 \pm 0.15$ \\
\hline 9091.37 & $14.63 \pm 0.06$ & $8.11 \pm 0.45$ \\
\hline 9095.69 & $14.61 \pm 0.02$ & $8.21 \pm 0.15$ \\
\hline 9097.90 & $14.54 \pm 0.02$ & $8.56 \pm 0.16$ \\
\hline 9098.75 & $14.65 \pm 0.04$ & $8.02 \pm 0.30$ \\
\hline 9098.98 & $14.50 \pm 0.02$ & $8.78 \pm 0.16$ \\
\hline 9099.43 & $14.58 \pm 0.06$ & $8.36 \pm 0.46$ \\
\hline 9099.69 & $14.53 \pm 0.02$ & $8.62 \pm 0.16$ \\
\hline 9099.70 & $14.53 \pm 0.02$ & $8.62 \pm 0.16$ \\
\hline 9099.81 & $14.50 \pm 0.02$ & $8.78 \pm 0.16$ \\
\hline 9100.40 & $14.51 \pm 0.06$ & $8.72 \pm 0.48$ \\
\hline 9100.70 & $14.53 \pm 0.02$ & $8.62 \pm 0.16$ \\
\hline 9101.73 & $14.45 \pm 0.06$ & $9.05 \pm 0.50$ \\
\hline 9101.76 & $14.47 \pm 0.02$ & $8.94 \pm 0.16$ \\
\hline 9101.89 & $14.47 \pm 0.02$ & $8.94 \pm 0.16$ \\
\hline 9102.38 & $14.54 \pm 0.06$ & $8.56 \pm 0.47$ \\
\hline 9104.37 & $14.53 \pm 0.01$ & $8.62 \pm 0.08$ \\
\hline 9104.75 & $14.42 \pm 0.02$ & $9.23 \pm 0.17$ \\
\hline 9105.35 & $14.43 \pm 0.01$ & $9.17 \pm 0.08$ \\
\hline 9107.55 & $14.45 \pm 0.06$ & $9.05 \pm 0.50$ \\
\hline 9114.59 & $14.42 \pm 0.06$ & $9.23 \pm 0.51$ \\
\hline $\begin{array}{l}9120.86 \\
9121.39\end{array}$ & $\begin{array}{l}14.38 \pm 0.04 \\
14.41 \pm 0.03\end{array}$ & $\begin{array}{l}9.46 \pm 0.35 \\
9.29 \pm 0.26\end{array}$ \\
\hline 9121.69 & $14.31 \pm 0.02$ & $9.90 \pm 0.18$ \\
\hline 9121.79 & $14.24 \pm 0.02$ & $10.37 \pm 0.19$ \\
\hline 9124.69 & $14.31 \pm 0.02$ & $9.90 \pm 0.18$ \\
\hline 9127.85 & $14.31 \pm 0.03$ & $9.90 \pm 0.27$ \\
\hline 9131.38 & $14.31 \pm 0.06$ & $9.90 \pm 0.55$ \\
\hline 9133.66 & $14.42 \pm 0.02$ & $9.23 \pm 0.17$ \\
\hline 9146.30 & $14.42 \pm 0.06$ & $9.23 \pm 0.51$ \\
\hline 9148.32 & $14.49 \pm 0.06$ & $8.83 \pm 0.49$ \\
\hline 9149.33 & $14.55 \pm 0.01$ & $8.51 \pm 0.08$ \\
\hline 9149.37 & $14.54 \pm 0.06$ & $8.56 \pm 0.47$ \\
\hline 9150.36 & $14.51 \pm 0.06$ & $8.72 \pm 0.48$ \\
\hline 9154.69 & $14.61 \pm 0.02$ & $8.21 \pm 0.15$ \\
\hline 9159.37 & $14.60 \pm 0.02$ & $8.26 \pm 0.15$ \\
\hline 9162.35 & $14.58 \pm 0.01$ & $8.36 \pm 0.08$ \\
\hline 9213.62 & $14.45 \pm 0.02$ & $9.05 \pm 0.17$ \\
\hline 9214.65 & $14.42 \pm 0.02$ & $9.23 \pm 0.17$ \\
\hline 9215.66 & $14.40 \pm 0.02$ & $9.34 \pm 0.17$ \\
\hline
\end{tabular}




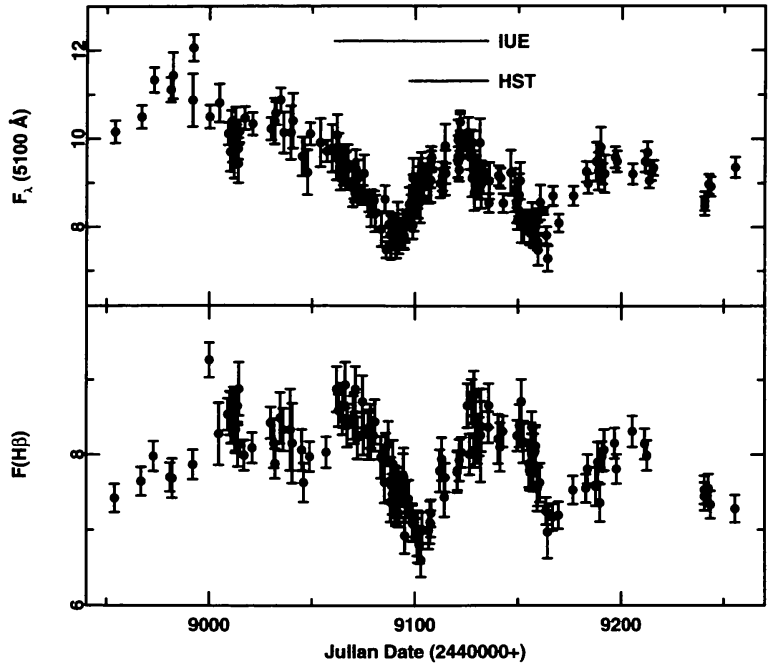

FIG. 12.-Continuum fluxes at $5100 \AA$ (top panel) and $\mathrm{H} \beta$ emissionline fluxes (bottom panel) for NGC 5548, as given in Tables 21 and 23 , from 1992 November through 1993 September. Fluxes are in the rest frame of NGC 5548, and are in units of $10^{-15}$ ergs s $\mathrm{cm}^{-1} \AA^{-1}$ for the continuum and $10^{-13} \mathrm{ergs} \mathrm{s}^{-1} \mathrm{~cm}^{-2}$ for the line. The periods during which NGC 5548 was monitored by IUE (UT 1993 March 19-1993 May 27) and by HST (UT 1993 April 19-1993 May 27) are also shown.

conservative approach is to reject a priori those points that could clearly bias the result.

Note the general characteristics of the FOS continuum light curves shown in Figure 6: an initial rise of $\sim 50 \%$ during the first 10 days is followed by a small decrease. This is followed by another increase of $\sim 20 \%$ over the next 4 days and then a similar decrease. Following a span of 4 days (JD 2,449,114-JD $2,449,117)$ during which the continuum was apparently relatively inactive, an increase of $\sim 30 \%$ occurred over about 5 days, followed by a monotonic decrease to nearly the level at the start of the HST campaign. Both $I U E$ and ground-based observations recorded a large decrease in the continuum just prior to the $H S T$ campaign (Figs. 10 and 12). It is apparent that the continuum reached a minimum just before the $H S T$ campaign began. The dynamic range in the continuum variations was significantly smaller during the combined HST/IUE campaign $\left(R_{\max }=2.5\right.$ at $\left.1350 \AA\right)$ than in the $1988-1989$ campaign $\left(R_{\max }=4.5\right.$ at $1350 \AA$; Paper I $)$.

Inspection of Figure 6 and Table 24 shows that the amplitude of variability appears to decrease with increasing continuum-band wavelength, just as was seen in 1988-1989 (Paper I). It is also apparent in Figure 6 that the small-scale features in the light curves (local maxima and minima) become less distinct or more smeared out at longer wavelengths. At least some of this effect may be due to dilution from some combination of the wings of very broad emission lines, the Balmer continuum, the Fe II pseudo-continuum, etc. However, some part of the effect may also be intrinsic to the continuum source. If this can be further substantiated, it is potentially important to our understanding of the nature of the continuum.

As in the 1988-1989 campaign, the high-ionization emission lines, He II and $\mathrm{N} v$, underwent the largest amplitude variations (see Figs. 8-11 and $F_{\text {var }}$ and $R_{\max }$ in Table 24), while the variations were smallest in the lower ionization lines of the $\mathrm{C}$ III ] complex. The $\mathrm{C}$ IV core and total fluxes had similar fluctuation amplitudes, in both cases larger than that of the C III] complex. The Ly $\alpha$ core showed fluctuations larger than seen in $\mathrm{C}$ IV, and the total Ly $\alpha$ line flux showed even larger fluctuations. The variability in the Si IV + O IV ] complex was intermediate between the high-ionization lines and Ly $\alpha$.

The light curves of the high-ionization lines mimicked the character of the continuum variations as described above. The true fluctuations in $\mathrm{N} \mathrm{v}$ were almost certainly larger than indicated here, since the $\mathrm{N} v$ direct-integration measurement includes a contribution from the Ly $\alpha$ wing. The FOS C IV and Ly $\alpha$ light curves were nearly "ramps" (i.e., monotonically increasing functions of time), before they flattened and possibly started to turn down at the end of the campaign. These two lines, at least in total flux, responded mainly to the longer timescale, larger amplitude continuum variations (a timesmoothed version of the continuum light curve during the $H S T$ campaign shows only a rise and a fall). The C IV and Ly $\alpha$ emission lines did not respond strongly to the shorter timescale, smaller amplitude continuum variations. This behavior is consistent with the average 6-10 day response times obtained in the 1988-1989 campaign. The HST campaign was not quite long enough to sample adequately the total-flux variations in these two emission lines.

Figure 8 shows that the $\mathrm{C}$ III] complex also responded to the continuum variations in a monotonically increasing fashion; however, during the first 6 days of the campaign, the flux in this blended feature underwent a small but steady decrease. This decrease may have been the result of the large decrease in the continuum recorded by the IUE and ground-based observations just prior to the $H S T$ campaign. If so, then one may infer an approximate lag of 17 days, which is consistent with the results reported in Paper I. Unfortunately, the poor quality of the SWP spectra in this region renders more detailed analysis of the $\mathrm{C}$ III] response rather uncertain.

\subsection{Characteristics of the Optical Database}

The optical data span a total of 286 days. The sampling characteristics and variability parameters for this fifth year of our optical monitoring program are summarized in Table 24; these can be compared directly with the results for previous years, as given in Table 6 of Paper VII. Neither of the variability parameters, $F_{\text {var }}$ and $R_{\max }$, has been adjusted for the effects of nonvarying components, such as the stellar continuum or the $\mathrm{H} \beta$ narrow line. The sampling and variability parameters for the optical database are also given separately for the periods corresponding to the $I U E$ and $H S T$ monitoring campaigns. It is notable that during year 5 of this monitoring program NGC 5548 showed less variability (as characterized by $F_{\mathrm{var}}$ and $R_{\max }$ ) in the optical continuum and $\mathrm{H} \beta$ emission line than in any of the previous years.

\subsection{Cross-Correlation Results}

As in our previous papers, we have employed two separate cross-correlation methods in a preliminary time-series analysis of the continuum and emission-line variability. The interpolation cross-correlation function (CCF; cf. Gaskell \& Sparke 1986; Gaskell \& Peterson 1987) and the discrete correlation 
TABLE 24

VARIABILITY PARAMETERS AND SAMPLING CHARACTERISTICS

\begin{tabular}{|c|c|c|c|c|c|c|}
\hline \multirow[b]{2}{*}{$\begin{array}{c}\text { Feature } \\
\text { (1) }\end{array}$} & \multirow{2}{*}{$\begin{array}{l}\text { Number } \\
\text { of Epochs } \\
(2)\end{array}$} & \multicolumn{2}{|c|}{ Sampling } & \multirow{2}{*}{$\begin{array}{c}\text { Mean } \\
\text { Flux } \\
(5) \\
\end{array}$} & \multirow[b]{2}{*}{$\begin{array}{c}F_{\text {var }} \\
(6)\end{array}$} & \multirow[b]{2}{*}{$\begin{array}{c}R_{\max } \\
(7)\end{array}$} \\
\hline & & $\begin{array}{c}\text { Average } \\
(3)\end{array}$ & $\begin{array}{c}\text { Median } \\
(4)\end{array}$ & & & \\
\hline$F_{\lambda}(1145 \AA) H S T$ only $\ldots \ldots \ldots \ldots$ & 39 & 1.00 & 1.00 & $3.83^{a}$ & 0.167 & 2.05 \\
\hline$F_{\lambda}(1350 \AA) H S T$ only $\ldots \ldots \ldots \ldots$ & 39 & 1.00 & 1.00 & $3.60^{a}$ & 0.139 & 1.86 \\
\hline$F_{\lambda}(1460 \AA) H S T$ only $\ldots \ldots \ldots \ldots$ & 39 & 1.00 & 1.00 & $3.26^{a}$ & 0.143 & 1.83 \\
\hline$F_{\lambda}(1790 \AA) H S T$ only $\ldots \ldots \ldots \ldots$ & 39 & 1.00 & 1.00 & $2.74^{a}$ & 0.122 & 1.71 \\
\hline$F_{\lambda}(2030 \AA) H S T$ only $\ldots \ldots \ldots$ & 39 & 1.00 & 1.00 & $2.17^{a}$ & 0.117 & 1.62 \\
\hline$F_{\lambda}(2195 \AA) H S T$ only $\ldots \ldots \ldots$ & 39 & 1.00 & 1.00 & $1.99^{a}$ & 0.109 & 1.53 \\
\hline Ly $\alpha \lambda 1216$ core $H S T$ only ....... & 39 & 1.00 & 1.00 & $410 .^{b}$ & 0.120 & 1.49 \\
\hline Ly $\alpha \lambda 1216$ total $H S T$ only . ....... & 39 & 1.00 & 1.00 & $718^{b}$ & 0.127 & 1.61 \\
\hline $\mathrm{N} \vee \lambda 1240 H S T$ only $\ldots \ldots \ldots \ldots \ldots$ & 39 & 1.00 & 1.00 & $82.2^{b}$ & 0.130 & 1.75 \\
\hline Si IV + O IV] $\lambda 1402 H S T$ only .... & 39 & 1.00 & 1.00 & $77.6^{b}$ & 0.129 & 1.65 \\
\hline C IV $\lambda 1549 H S T$ only ............ & 39 & 1.00 & 1.00 & $670 .^{b}$ & 0.090 & 1.37 \\
\hline $\mathrm{He}$ II $+\mathrm{O}_{\text {III }]} H S T$ only $\ldots \ldots \ldots \ldots$ & 39 & 1.00 & 1.00 & $89.3^{b}$ & 0.140 & 1.82 \\
\hline $\mathrm{C} \mathrm{III}]+\mathrm{Si} \mathrm{III}] H S T$ only $\ldots \ldots \ldots \ldots$ & 39 & 1.00 & 1.00 & $128 .^{b}$ & 0.085 & 1.32 \\
\hline$F_{\lambda}(1350 \AA)$ Combined IUE \& HST & 53 & 1.43 & 1.01 & $3.38^{a}$ & 0.187 & 2.50 \\
\hline Ly $\alpha \lambda 1216$ Combined IUE \& HST & 53 & 1.43 & 1.01 & $699 .^{b}$ & 0.146 & 1.76 \\
\hline C IV $\lambda 1549$ Combined IUE \& HST & 53 & 1.43 & 1.01 & $622 .^{b}$ & 0.109 & 1.53 \\
\hline \multicolumn{7}{|l|}{$F_{\lambda}(5100 \AA)$} \\
\hline $\begin{array}{l}\text { Year } 5 \text { (1992 Nov - } 1993 \mathrm{Sep}) \\
\text { IUE campaign (1993 Mar } 19\end{array}$ & 192 & 1.58 & 0.77 & $0.906^{a}$ & 0.093 & 1.65 \\
\hline $\begin{array}{l}-1993 \text { May } 27) \ldots \ldots \ldots \ldots \ldots \\
\text { HST campaign (1993 Apr } 19\end{array}$ & 97 & 0.73 & 0.51 & $0.881^{a}$ & 0.071 & 1.39 \\
\hline - 1993 May 27) .............. & 59 & 0.67 & 0.37 & $0.914^{a}$ & 0.050 & 1.29 \\
\hline \multicolumn{7}{|l|}{$\mathrm{H} \beta$} \\
\hline Year 5 (1992 Nov - 1993 Sep) .. & 142 & 2.14 & 0.99 & $79.3^{b}$ & 0.064 & 1.40 \\
\hline
\end{tabular}

a In units of $10^{-14} \mathrm{ergs}^{-1} \mathrm{~cm}^{-2} \AA^{-1}$

${ }^{b}$ In units of $10^{-14} \mathrm{ergs}^{-1} \mathrm{~cm}^{-2}$.

function (DCF; cf. Edelson \& Krolik 1988) are computed for various pairs of light curves as described by White \& Peterson (1994). The results are shown in Figures 13-17 and tabulated in Table 25 . The parameter $\Delta t_{\text {peak }}$ is the location in days of the peak of the CCF, which has value $r_{\max }$. Also given in Table 25 is the value of the centroid $\Delta t_{\text {centroid }}$ (in days) of the CCF, which is computed using all points near the peak of the CCF with amplitudes greater than $0.5 r_{\max }$. (This is sometimes referred to as the centroid at the $50 \%$ level.)

In Figure 13 we present the correlation results for the FOS data alone. The DCF bin width is 1 day. In each panel we show the result of cross-correlating the $1350 \AA$ continuum light curve with the light curve designated in the upper left-hand corner; note that a positive time delay means that the variations in the feature designated in each panel lag behind the variations in the $1350 \AA$ continuum. In the case of the optical $5100 \AA$ continuum, only those points which fall within the time span of the HST campaign are included in the CCF calculation. The UV/optical-continuum cross-correlation function is nearly symmetric about a small positive delay ( see Table 25 ). As explained earlier, the temporal coverage of the optical data fell off substantially during the second half of the $H S T$ campaign, which makes these results somewhat uncertain. Cross-correlations of all other FOS continuum bands with the $5100 \AA$ continuum produce virtually identical results. Crosscorrelations between various of the FOS continuum bands have high values of $r_{\max }(\gtrsim 0.96)$ and yield very small delays that are consistent with zero $(|\tau| \approx 0.2$ days $)$. As expected, the high-ionization lines have well-resolved cross-correlation peaks, while the Si IV + O IV ], C IV, and Ly $\alpha$-core cross-correlations have progressively less well resolved peaks. This is consistent with what we know about the approximate mean response times of these emission lines, which were derived during the first 1988-1989 campaign (Paper I), in combination with the observed character of the continuum variations during the HST campaign.

In Figure 14 we present the cross-correlation results obtained by using the combined ultraviolet data set. Again, the cross-correlations for the light curves shown in the upper lefthand corner of each panel are computed relative to the $1350 \AA$ continuum. The DCF bin width is 2 days in each case. The peak and centroid of the $5100 \AA$ continuum CCF are both positive with values $\sim 1$ day, consistent with the FOS result. However, the uncertainty in this value is also $\sim 1$ day, which is onehalf the SWP temporal resolution. The cross-correlation function is slightly asymmetric toward positive lags. On account of the longer time coverage and the relatively deep continuum minimum around JD 2,449,090, both the Ly $\alpha$ and the C IV responses are resolved in the combined ultraviolet data set. These lines tracked the large decline and then recovery in the continuum before and during the HST campaign, respectively. Their responses lagged behind the $1350 \AA$ continuum by $\sim 7$ days, which is consistent with the results presented in Paper I.

In Figure 15 and Table 25 we present the cross-correlation 

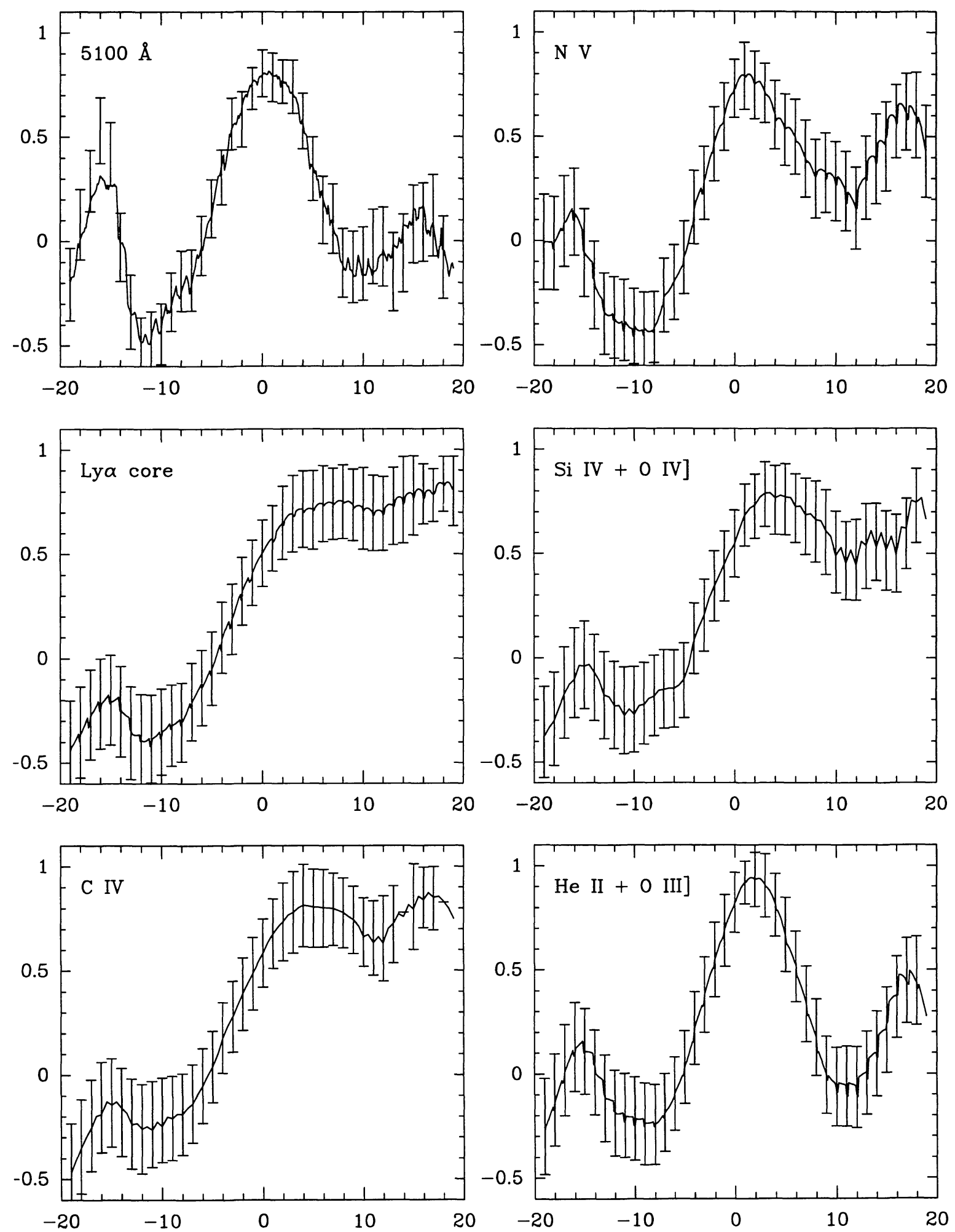

FIG. 13.--Interpolated cross-correlation ( solid curves) and discrete correlation functions (with error bars) of the $1350 \AA$ A continuum with the overlapping $5100 \AA$ continuum and the major ultraviolet emission-line measurements during the $H S T$ campaign. The units on the axes are correlation coefficient (ordinate) and delay in days (abscissa). 

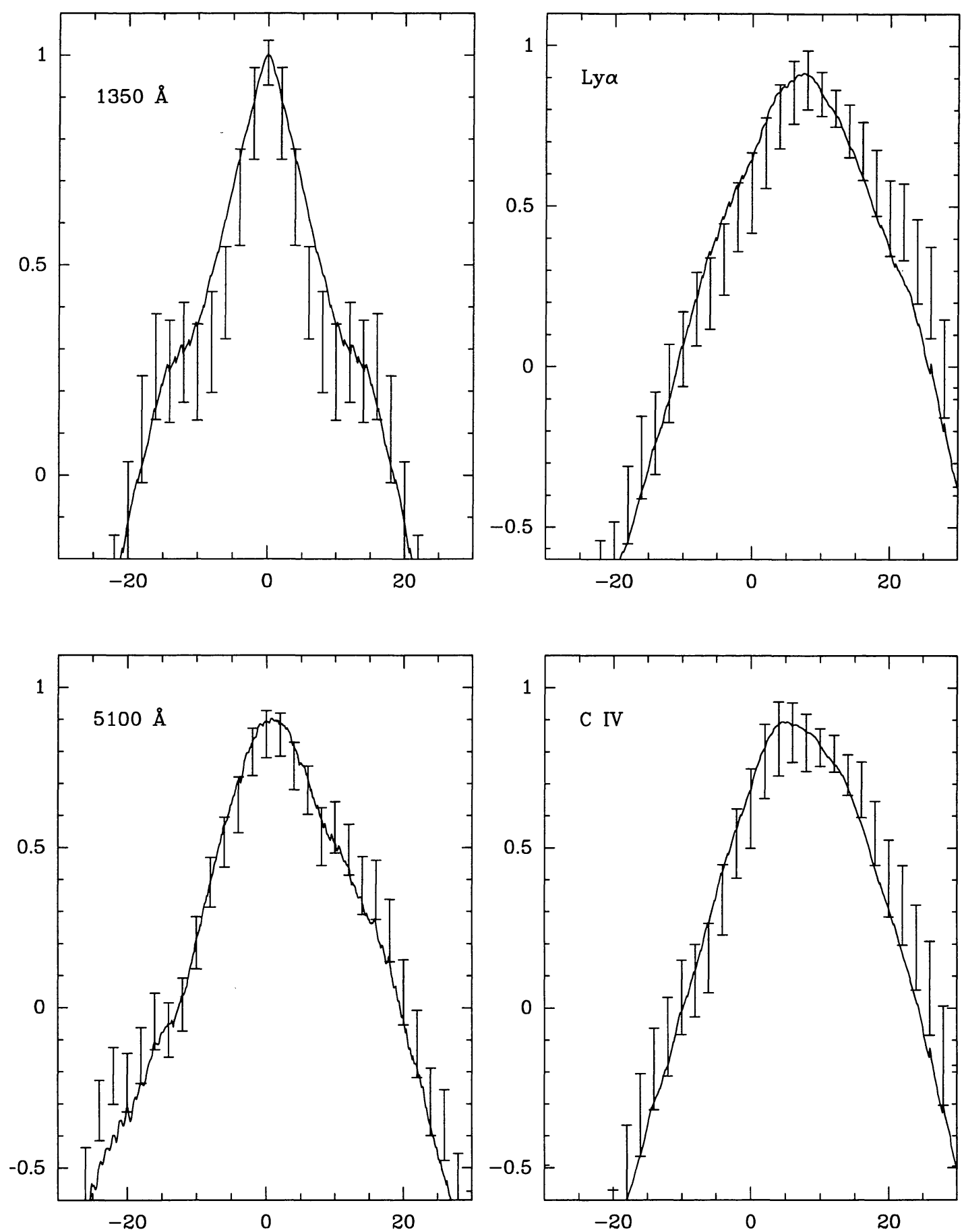

FIG. 14.-Interpolated cross-correlation (solid curves) and discrete correlation functions (with error bars) of the $1350 \AA$ continuum with itself (its autocorrelation) and with the overlapping $5100 \AA$ continuum and the Ly $\alpha$ and C IV emission lines for the "combined" ultraviolet data set. The units on the axes are correlation coefficient (ordinate) and delay in days (abscissa).

results for the $\mathrm{C}$ IV emission-line profile components. The four upper panels show the cross-correlation functions for the specified C IV component light curves relative to the $1350 \AA$ light curve. Of the four profile components, only the red wing has a resolved response to the continuum variability. All of the crosscorrelation functions show the same gross characteristics, namely, that they increase with increasing time delay but begin to level off at positive delays of several days. Figure 9 shows that, unlike any of the other components, the red wing rose rapidly in the first 11 days of the $H S T$ campaign before it began to level off (by JD 2,449,110). In contrast, the blue and red cores did not appear to level off until about JD 2,449,124; the light curves for the blue and red cores are very similar to one another, except that the red core rises slightly more rapidly in 

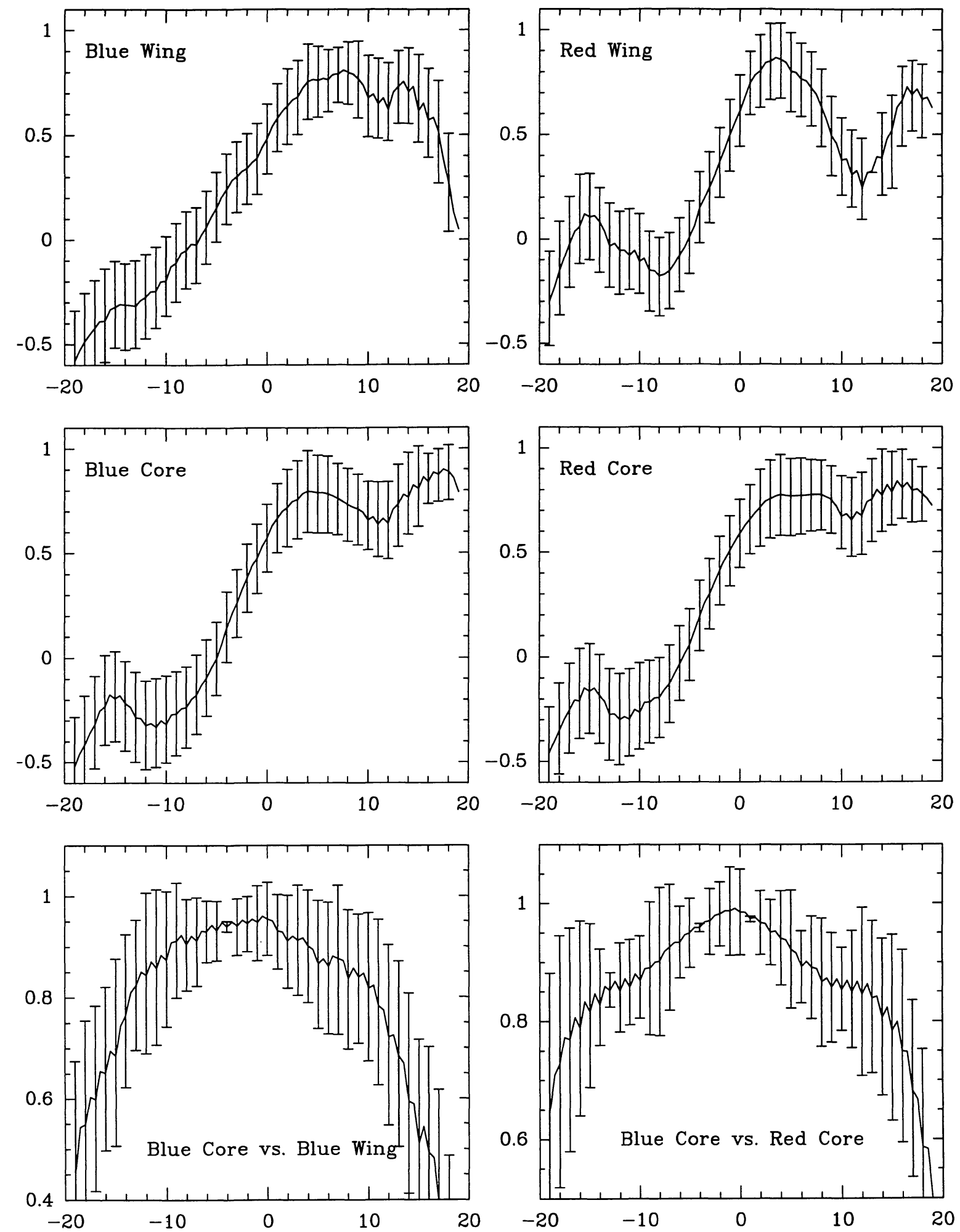

FIG. 15.-Interpolated cross-correlation (solid curves) and discrete correlation functions (with error bars) of the $1350 \AA$ continuum with the C IV emission-line profile components measured during the HST campaign. The units on the axes are correlation coefficient (ordinate) and delay in days (abscissa). 


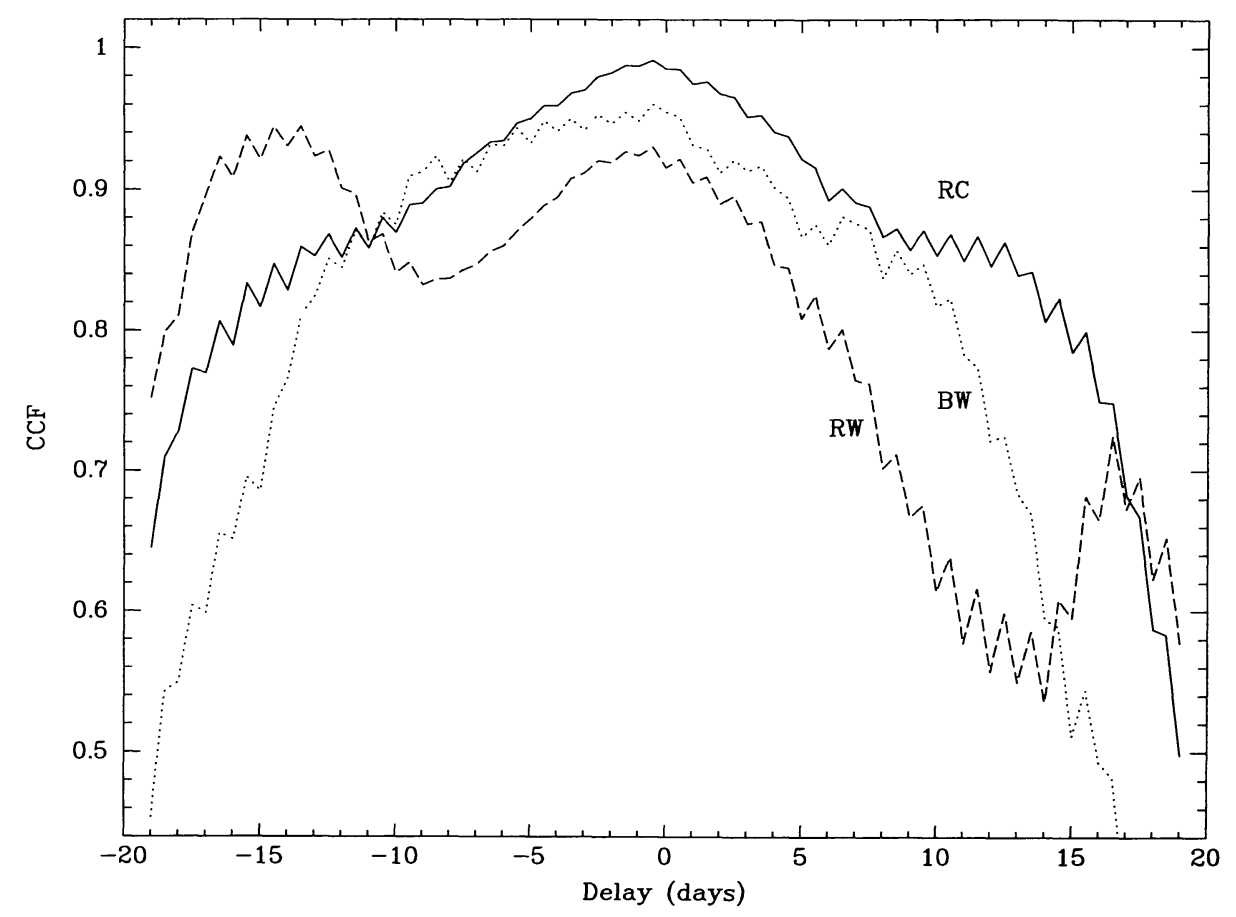

FIG. 16.-Close-up comparison of the interpolated cross-correlation functions of the C IV blue core vs. blue wing ("BW"; dotted line) with that of the blue core vs. red core ("RC"; solid line) and the blue core vs. red wing ("RW"; dashed line). The red core CCF is centered near zero delay and is slightly asymmetric toward negative lag. These effects are somewhat more pronounced for the blue wing and blue core.

the first 11 days of the campaign. We suspect that at least some of the more rapid response of the red wing of $\mathrm{C}$ IV is attributable to contamination by $\mathrm{He}$ II, which responds much more rapidly than C IV. This can be investigated by detailed analysis of the profile variations, which is beyond the scope of the current paper and will be discussed elsewhere.

Small differences in the response of the various C IV components can be accentuated by direct cross-correlation of the component light curves; this reduces the effect of their firstorder similarity. The results of direct cross-correlation of the blue-core and red-core light curves are given in Table 25 and in the lower right-hand panel of Figure 15. The blue-core/redcore cross-correlation function is very nearly symmetric about a delay near zero, with a slight asymmetry toward negative delays. The lower left-hand panel in Figure 15 shows the result of cross-correlating the blue-core and blue-wing light curves. The cross-correlation function shows a relatively strong asymmetry in the sense that the variations in the blue wing appear to lead the corresponding variations in the blue core, as might be concluded by direct comparison of the light curves (Fig. 9) in which it is seen that the rise in the blue-wing flux levels off somewhat before the corresponding rise in the blue-core flux. In Figure 16 we show a direct comparison of the blue-core/ red-core CCF and the blue-core/blue-wing CCF which suggests that the blue wing leads the two core components by a very small amount. Also shown is the blue-core/red-wing $\mathrm{CCF}$, which as expected shows the strongest asymmetry to negative delays (i.e., the red wing leading the blue core). In summary, the red wing is the only C IV component that we can confidently state varies differently from the other components; however, the importance of He II contamination needs to be studied in more detail before any conclusions can be drawn about the BLR velocity field. There is a weak suggestion that the blue wing responds slightly faster than the blue core, and more complete analysis may determine whether this is real.

The top panels in Figure 17 and Table 25 show the result of cross-correlating the $5100 \AA$ and $\mathrm{H} \beta$ light curves for the entire fifth year, from 1992 November to 1993 September. The H $\beta$ emission-line variations lag behind the optical continuum variations by about 2 weeks; the $\mathrm{H} \beta$ lag is approximately twice as large as the Ly $\alpha$ or CIV lags, which is what was found in the 1988-1989 campaign (Papers I and II). The values of the peak and centroid of the CCF for year 5 are somewhat smaller than those obtained in year 1 (1988-1989), but are fairly consistent with the differences found for other years (Paper VII). The lower panel in Figure 17 shows the optical continuum autocorrelation function and the optical sampling window autocorrelation function. That the latter function is much narrower than the former is an indication that most of the important variations were resolved in the ground-based campaign. Table 26 lists the FWHMs of the autocorrelation functions for the 1350 and $5100 \AA$ continuum light curves.

\subsection{General Discussion}

As described in $\S 1$, this program was undertaken with the primary objective of addressing three specific questions:

1. What is the lag between the UV and the optical continuum variations? 


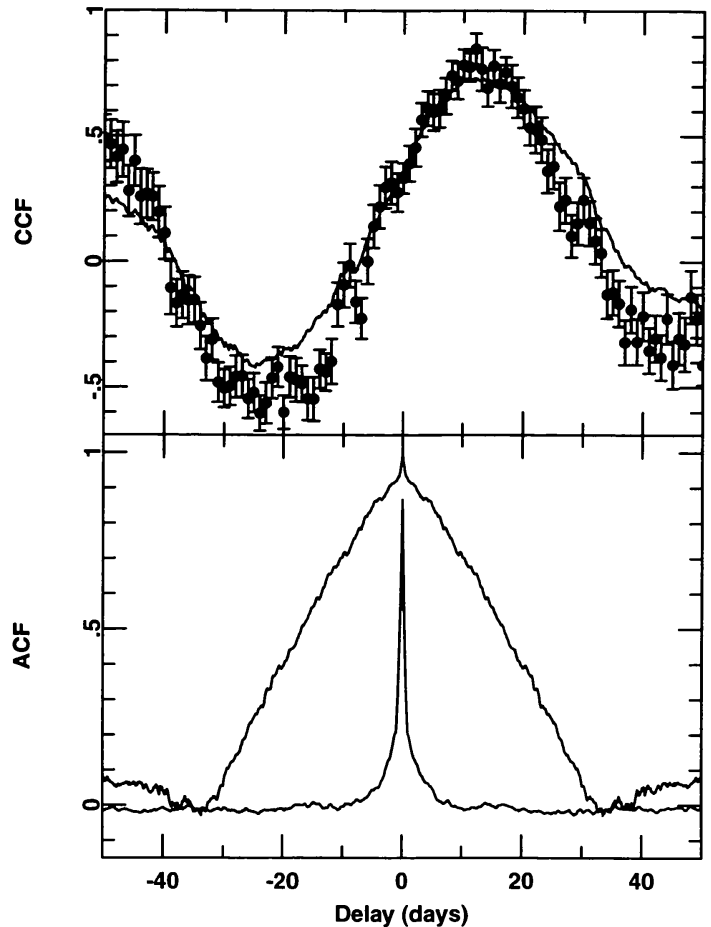

FIG. 17.-Shown in the top panel are the cross-correlation functions for the optical continuum ( $5100 \AA)$ and the $\mathrm{H} \beta$ emission line for the light curves shown in Fig. 12. The interpolation CCF is shown as a smooth line, and the DCF values are plotted as individual points with associated uncertainties. The bin width for the DCF is 1 day. The corresponding continuum autocorrelation function and the sampling window autocorrelation function (which, as described by Gaskell \& Peterson 1987, illustrates the effect of interpolating the data between observations) are shown in the lower panel.

2. What is the lag for the highest ionization lines?

3. What is the velocity field of the CIV -emitting region?

These observations more firmly establish our earlier finding (Papers I and II) that any possible lag between the UV and optical continuum variations is indeed small. By using various subsets of the ultraviolet data obtained in this experiment, we consistently find that the optical continuum lags behind the UV continuum by about 1 day or less. In order to better establish the upper limit on a possible lag between the UV and optical continua, we performed a series of simple Monte Carlo simulations in order to assess the uncertainty in the cross-correlation result. These calculations were done by using the 1350 $\AA$ continuum measurements from the combined ultraviolet data set to model the continuum behavior. For each Monte Carlo realization, the following procedure was followed:

1. A model UV continuum light curve was produced from the observations by altering each flux measurement under the assumption that the quoted errors are distributed normally. Random Gaussian deviates were used to alter the flux at each point. This continuum was then linearly interpolated from point to point as necessary.

2. A model optical continuum light curve was generated by shifting the (noise-free) model UV continuum in time; in other words, we made the very simple assumption that the optical continuum is simply a time-delayed version of the UV
TABLE 25

CROSS-CORRELATION RESULTS

\begin{tabular}{|c|c|c|c|c|}
\hline $\begin{array}{c}\text { First } \\
\text { Series } \\
(1)\end{array}$ & $\begin{array}{c}\text { Second } \\
\text { Series } \\
(2)\end{array}$ & $\begin{array}{c}\Delta t_{P e a k} \\
\text { (days) } \\
\text { (3) }\end{array}$ & $\begin{array}{c}\Delta t_{\text {Centrotd }} \\
\text { (days) } \\
\text { (4) }\end{array}$ & $\begin{array}{c}r_{\max } \\
(5)\end{array}$ \\
\hline \multicolumn{5}{|c|}{$H S T$ FOS measurements only: } \\
\hline$F_{\lambda}(1350 \AA)$ & $F_{\lambda}(5100 \AA)$ & 0.6 & 0.6 & 0.81 \\
\hline$F_{\lambda}(1350 \AA)$ & $\mathrm{N} \vee \lambda 1240$ & 1.4 & 2.4 & 0.80 \\
\hline$F_{\lambda}(1350 \AA)$ & $\mathrm{He} \mathrm{II}+\mathrm{OIII}]$ & 1.7 & 1.8 & 0.94 \\
\hline$F_{\lambda}(1350 \AA)$ & Si IV + O IV] & 3.5 & $4.8^{a}$ & 0.79 \\
\hline$F_{\lambda}(1350 \AA)$ & Civ blue wing & 7.5 & 8.3 & 0.81 \\
\hline$F_{\lambda}(1350 \AA)$ & C IV red wing & 3.5 & 4.3 & 0.87 \\
\hline C Iv blue core & Civ blue wing & -0.4 & -1.2 & 0.96 \\
\hline $\mathrm{C}$ IV blue core & Civ red core & -0.5 & -0.9 & 0.99 \\
\hline \multicolumn{5}{|c|}{ Combined IUE SWP \& HST FOS measurements: } \\
\hline$F_{\lambda}(1350 \AA)$ & $F_{\lambda}(5100 \AA)$ & 0.7 & $1.2^{b}$ & 0.90 \\
\hline$F_{\lambda}(1350 \AA)$ & Ly $\alpha \lambda 1216$ & 7.5 & 6.9 & 0.92 \\
\hline$F_{\lambda}(1350 \AA)$ & Civ $\lambda 1549$ & 4.6 & 7.0 & 0.90 \\
\hline \multicolumn{5}{|c|}{ Ground-based measurments only: } \\
\hline$F_{\lambda}(5100 \AA)$ & $F(\mathrm{H} \beta)$ & 10.6 & 14.7 & 0.7 \\
\hline
\end{tabular}

${ }^{a}$ Centroid measured at $80 \%$ level.

${ }^{\text {b }}$ Centroid measured at $80 \%$ level. The centroid delay at the $50 \%$ level is 2.0 days.

continuum. We then added a constant component to the model optical continuum (to represent the starlight component) and diluted the amplitude of variation to achieve approximate consistency with the observed amplitude of variation in the optical continuum.

3. The model optical continuum light curve was then sampled to obtain the same number of data points as in the optical campaign during the combined ultraviolet monitoring campaign. The optical points were sampled in such a way as to preserve the distribution of intervals between observations. Again, random Gaussian deviates were applied to the sampled points to simulate observational errors.

4. The UV and optical model sample points were then cross-correlated, and the value of $\Delta t_{\text {peak }}$ was recorded.

The above procedure was repeated many times to build up a probability distribution for $\Delta t_{\text {peak }}$ as a function of the time shift between the UV and optical continua. The principal result of these simple simulations is that we can conservatively estimate that the probability of obtaining a lag of 0.7 days or less (i.e., the experimental value) is less than $\sim 0.1$ if the actual shift

TABLE 26

CONTINUUM AUTOCORRELATION RESULTS

\begin{tabular}{|c|c|}
\hline Continuum Band & $\begin{array}{c}\text { FWHM } \\
\text { (days) }\end{array}$ \\
\hline$F_{\lambda}\left(1350 \AA{ }_{0}\right)^{\mathrm{a}} \ldots \ldots \ldots$ & 6.7 \\
\hline$F_{\lambda}\left(1350 \AA{ }^{\circ}\right)^{b} \ldots \ldots \ldots$ & 15.3 \\
\hline$F_{\lambda}(5100 \AA)^{\mathrm{c}} \ldots \ldots \ldots$ & 34.0 \\
\hline$\hat{F_{\lambda}}(5100 \AA)^{\mathrm{d}} \ldots \ldots \ldots$ & 8.4 \\
\hline \multicolumn{2}{|c|}{$\begin{array}{l}\text { a During } H S T \text { campaign. } \\
\text { b During combined } I U E, H S T \text { cam- } \\
\text { aign. } \\
\text { c Year } 5 . \\
{ }^{d} \text { During } H S T \text { campaign. }\end{array}$} \\
\hline
\end{tabular}


between the UV and optical continua is as large as 1.2 days. In other words, to the extent that these Monte Carlo simulations are valid, we can state with $\sim 90 \%$ confidence that the lag between the UV and optical continua is no larger than 1.2 days.

The lags for the highest ionization lines (He II $\lambda 1640$ and $\mathrm{N} v \lambda 1240$ ) are measured to be slightly less than 2 days, but are decidedly nonzero. Evaluation of the uncertainties in these lags is somewhat problematic because the uncertainties depend on the shape of the transfer function. Uncertainties in the geometry of the line-emitting region are much larger than the formal uncertainties in the measurement of location of the CCF peak or centroid, which are $\sim 1$ day or less. The original 1988-1989 campaign suggested lags of $\sim 2$ days for $\mathrm{He}$ II and $\mathrm{N} \mathrm{v}$, but the poorer temporal sampling and lower signal-to-noise ratio of the original campaign, as well as a fixed-pattern artifact in the SWP camera that affects the He II region, left this result rather uncertain; in particular, the lags measured for the highest ionization lines in the original campaign were consistent with a lag of zero. The observations reported here thus have resulted in a marked improvement in determination of the $\mathrm{He}$ II and $\mathrm{N} v$ lags.

The results of the search for the velocity-dependent response of the $\mathrm{C}$ IV emission line are still quite ambiguous, although it seems clear that the kinematics of this region cannot be described in terms of pure radial motion, either infall or outflow. The response of the blue core $\left(-3000 \mathrm{~km} \mathrm{~s}^{-1} \leq \Delta v \leq 0 \mathrm{~km} \mathrm{~s}^{-1}\right)$ and the red core $\left(0 \mathrm{~km} \mathrm{~s}^{-1} \leq \Delta v \leq+3000 \mathrm{~km} \mathrm{~s}^{-1}\right)$ appear to be nearly identical, with no significant time delay between them. The red wing of $C$ IV responds decidedly more rapidly than the line cores, although the preliminary analysis here does not distinguish clearly between the possibilities of an infall component of the $\mathrm{CIV}$-emitting region and contamination by the blue wing of He II $\lambda 1640$. The preliminary analysis undertaken here also yields a weak suggestion that the blue-wing response is slightly more rapid than the response of the line cores, although the magnitude of this effect is barely discernible in the cross-correlation analysis and further investigation will be required before any degree of confidence can be ascribed to this finding. In any case, it is already clear that any velocitydependent line response is fairly subtle. There are indications that the $\mathrm{C}$ IV line profile changed in response to the most rapid continuum changes which occurred at the beginning of the HST campaign. Thus, some component of radial motion cannot yet be ruled out, and more detailed analysis of these observations will be required.

The observations reported here confirm the existence of an inverse correlation between ionization level and lag (Paper I), i.e., the lines characteristic of the most highly ionized gas respond most rapidly to continuum variations. The differences in the response of various lines show that the BLR has a range in radius of more than an order of magnitude, and that it has a radially stratified ionization structure.

\section{SUMMARY AND CONCLUSIONS}

We have described an intensive set of ultraviolet spectroscopic monitoring observations of NGC 5548 that were obtained with HST and IUE in 1993 March-May. This program was undertaken to address questions that required both higher signal-to-noise ratio and better time resolution than were achieved in our earlier IUE program in 1988-1989 on this same galaxy.

We also present ground-based optical observations covering the period 1992 November-1993 September. These data constitute a fifth year of coordinated ground-based coverage of variability in this source.

The acquisition and reduction of the space-based and ground-based data obtained are described in detail in this paper. While more extensive analysis and interpretation will appear in subsequent papers, here we have undertaken simple preliminary time-series analysis that allows us to reach some basic conclusions:

1. The UV and optical continua vary with little if any phase difference between them. Cross-correlation of the UV and optical continuum light curves shows that the optical continuum lags behind the UV continuum by $\sim 1$ day. Some simple Monte Carlo simulations indicate that the lag between the UV and optical continuum variations is less than 1.2 days at the $90 \%$ confidence level.

2. The variations of the highest ionization lines ( He II $\lambda 1640$ and $N$ V $\lambda 1240$ ) lag behind those of the UV continuum by $\sim 2$ days, with an uncertainty of $\sim 1$ day.

3. We have examined in a preliminary way the velocity-dependent response of the C IV $\lambda 1549$ emission line and find no evidence that the BLR kinematics involve predominantly radial motions. Neither infall nor outflow is indicated. We do find, however, admittedly weak indications that the higher radial velocity gas ( the line wings) responds somewhat more rapidly than the lower radial velocity gas (the line cores). The possibility that the line wings respond more rapidly than the line cores is suggestive of a virialized system, although it is not clear at this time whether the cloud motions are organized or random, or indeed what level of confidence can be ascribed to the result. The red wing of $C$ IV responds more rapidly than either the line core or the blue wing, but it is not clear how much of this might be ascribed to an infalling component of the C IV emitting region and how much might be due to contamination by the blue wing of $\mathrm{He}$ II $\lambda 1640$, which has a much shorter response time. There are also some indications that $\mathrm{C}$ IV profile variations occur on short timescales, apparently when the continuum is changing rapidly, and this could indicate that at least some BLR gas is in radial motion.

4. As in our earlier contributions on NGC 5548 and NGC 3783 , we find that the continuum appears to get "harder" as it gets brighter, i.e., the amplitude of variability is greater at shorter wavelengths. Furthermore, we find some indications that the longer wavelength continuum variations are somewhat smoother than those at shorter wavelengths, as though the highest temporal frequency variations have been filtered out of the longer wavelength continuum.

5. Our fifth complete year of monitoring the optical continuum and $\mathrm{H} \beta$ emission-line variations in NGC 5548 yields a response time that is about twice the response time for $\operatorname{Ly} \alpha$, as was found in Paper I. The peak of the optical continuum/ $\mathrm{H} \beta$ CCF is at a slightly smaller time delay ( $\sim 11$ days) than we have found for the other 4 years of optical monitoring (18-19 days; see Paper VII).

6. The observations reported here confirm that higher ionization lines respond to continuum variations more rapidly and with larger amplitude than lower ionization lines. 
We are deeply indebted to the entire FOS Instrument Team and Science Data Analysts, the $H S T$ scientists and engineers, and the Science Planning and Scheduling Branch for their patience and hard work. They share in the success of the HST portion of this project. K. T. K. spent valuable time with and especially wants to thank the following STScI staff members: Pierre Bely, Chris Blades, Howard Bushouse, John Fitch, Tony Keyes, Anne Kinney, Michael Rosa (ST/ECF), Peggy Stanley, Peter Stockman, Cindy Taylor, and Tom Wheeler. Extra special thanks go to Ralph Bohlin, who not only spent hours of his time in discussing the FOS flux calibration and related problems but also graciously made available the new FOS pipeline.

At the Goddard Space Flight Center we gratefully acknowledge the work of Cathy Imhoff, Joy Nichols, and their staff, responsible for running the project's SWP spectra through the NEWSIPS pipeline, and for answering our many inquiries into the NEWSIPS calibration.

We thank Richard Griffiths for kindly offering us the telescope pointing information from his parallel WFC observations.

We also wish to acknowledge the support of several project coinvestigators who felt that their contribution did not warrant inclusion on the list of authors: B. Balick, T. J. Balonek, P. D. Barthel, R. D. Blandford, T. J.-L. Courvoisier, R. M. Cutri, G. J. Ferland, P. M. Gondhalekar, D. Kazanas, C. F. McKee, E. J. A. Meurs, E. Pérez, A. Robinson, E. Rokaki, J. L. Sanz, T. Storchi-Bergmann, E. van Groningen, M. J. Ward, C.-C. $\mathrm{Wu}$, and $\mathrm{W}$. Zheng.

Finally, we thank R. Giacconi, H. S. Stockman, and R. E. Williams (STScI), Y. Kondo (IUE-GSFC), and the directors of the various ground-based observatories used in this program for their support of this demanding program.

We are pleased to acknowledge financial support of the spacebased component of this project by NASA through grants NAG51824 and STScI grant GO-3484.01-91A. We also acknowledge support for the ground-based observations through several agencies, including the following: National Science Foundation, grants AST-9112870 (Ohio State University), AST-8957063 (University of California, Berkeley); NASA Long-Term Space Astrophysics grants NAGW-2678 (K. H.) and NAGW-3315 (B. M. P.); BMFT grant Verbundforschung Astronomie DFG Ko 857/13-1 (Universitäts-Sternwarte Göttingen), ESO C\&EE Program for grant A-01-057 (Crimean Astrophysical Observatory); and Russian Basic Science Foundation grant 94-0204885a (N. G. B. and A. I. S.).

\section{APPENDIX A FOS BACKGROUND SUBTRACTION}

\section{A1. THE CHARGED-PARTICLE BACKGROUND}

A model for Cerenkov radiation background due to charged particles within the Earth's magnetic field was derived during Science Verification. The model is roughly quadratic in count rate across the diode array, with a minimum whose relative amplitude is $\sim 90 \%$ occurring near the center of the array; it is scaled by a constant which takes into account the spacecraft position in the Earth's magnetic field. However, the predicted scale factors have been recently shown to be deficient on average by $\sim 12 \%$ near low geomagnetic latitudes and by $\gtrsim 30 \%$ at high geomagnetic latitudes (Fitch \& Schneider 1993; Rosa 1993). A charged-particle background model with a multipole geomagnetic field is being derived at the time of this writing.

Assuming that the charged-particle background is the source of all counts in the zero-sensitivity region (a first-order approximation), one can derive the ratio of the background counts to object counts across the diode array. Even in the G130H spectra redward of geocoronal $\operatorname{Ly} \alpha$ this ratio was small, at most $\sim 0.07$, but it rose rapidly blueward (up to $\sim 0.5$ ) due to the plummeting detector sensitivity to direct first-order light. Although we cannot measure the $\mathrm{G} 190 \mathrm{H}$ background directly, if one assumes that it is higher by the same factor as in the G130H grating, then the maximum difference in the count rate in the G190H spectrum is $\lesssim 1 \%$. The much higher sensitivity with the $\mathrm{G} 190 \mathrm{H}$ grating minimizes the effects of the uncertainty in the background.

\section{A2. THE SCATTERED-LIGHT CONTRIBUTION TO THE BACKGROUND}

The blue-side detector of the FOS is sensitive to photons with wavelengths spanning $\sim 1137$ to $\sim 5500 \AA$. In principle, light from any of those wavelengths which scatters off the grating or off any irregularity along the entire optical path might produce spurious counts landing semirandomly across the diode array. Prelaunch experiments (Sirk \& Bohlin 1985) demonstrated the likely presence of scattered light within the FOS itself, and in particular the strong wavelength dependence of such in the G130H grating. Unfortunately, no scattered-light experiments were done during Science Verification. However, the present data, and those in the $H S T$ archive (Cunningham \& Caldwell 1993; Rosa 1993; Ayres 1994), demonstrate the presence of scattered light for the G130H grating, and certainly all others as well. A plot of the background count rate in the $\mathrm{G130H}$ grating measured in the zero-sensitivity region versus the object count rate, corrected for an estimate of the total background, shows a very strong correlation. (Additionally, spectra of stars and elliptical galaxies show unexpected ultraviolet upturns.)

As a consequence of these findings, scattered-light experiments were performed for all gratings and both sides of the FOS just prior to the first $H S T$ servicing mission of 1993 December on three stars ( spectral types M2 I, K5 III, and G2 V), where FOS data were to be compared to "solar blind" data from the Goddard High Resolution Spectrograph (GHRS) or the Hopkins Ultraviolet Telescope (HUT). Archival FOS versus GHRS or HUT data for objects of different spectral distributions were also to be analyzed. To first order, the relative amount of scattered light versus direct first-order light detected at the FOS diode array is related to the object's intrinsic spectral energy distribution as well as the detector's sensitivity. A recent analysis by Rosa (1994) finds that $\sim 90 \%$ of the scattered light is due to scattering off the ruled gratings. One effect is just the scattering of photons in random angles off the grating, due to irregularities in the rulings. Another effect is that the extreme wings $( \pm 1000 \AA)$ of the LSFs scatter zeroth-order and 


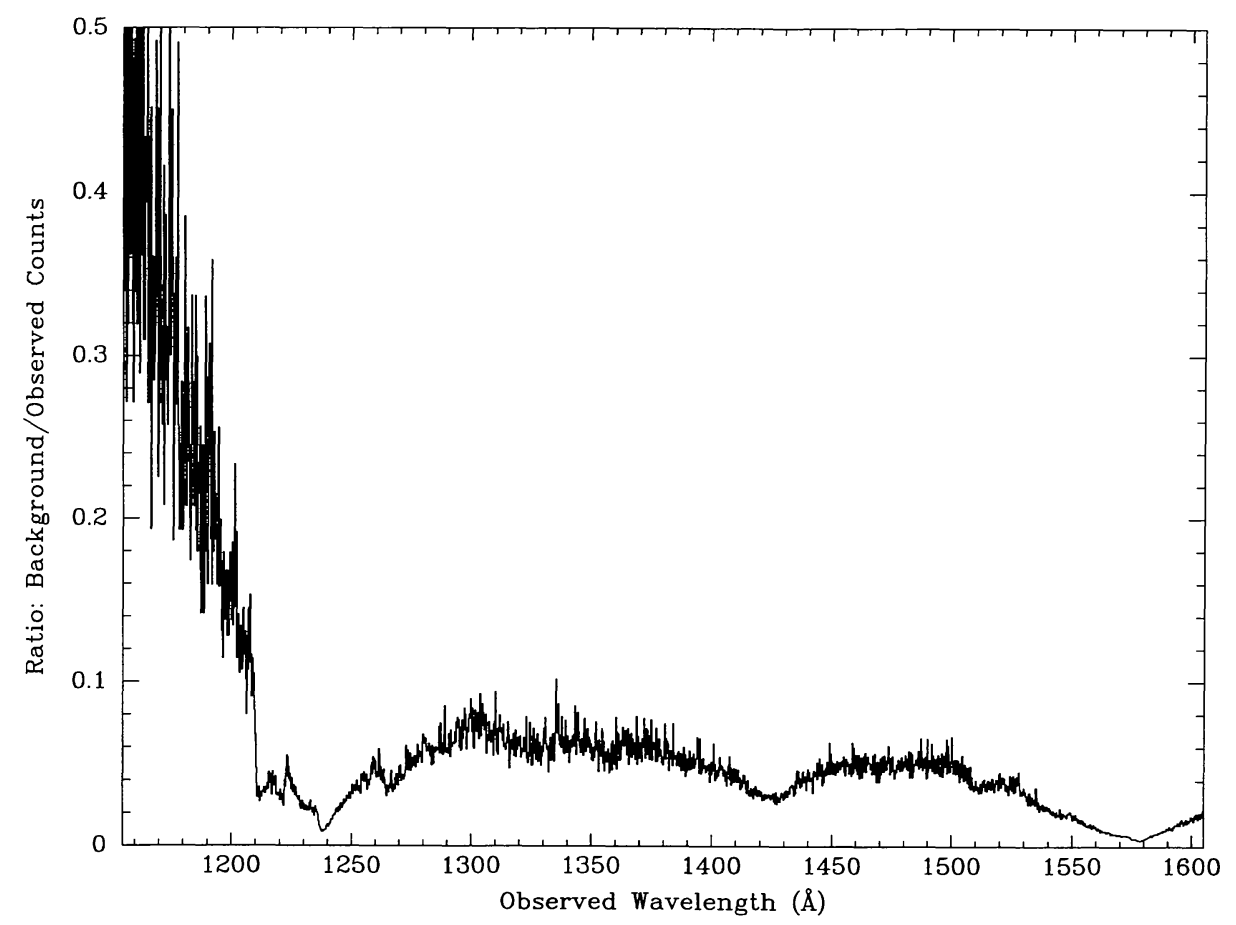

FIG. 18.-Ratio of the number of background counts to the total number of observed counts plotted as a function of wavelength for a G130H observation.

second-order light into first order. This scattered-light contribution begins to become important when the combination of the object's spectral energy distribution and the detector sensitivity falls off more rapidly with wavelength (diode) than the wings in the LSF. The shape of the scattered light "spectrum" is roughly flat, when the first-order light dominates over the scattered light. In the regime where scattered light overwhelms the first-order light, the spectrum takes on the shape of a combination of the wing of the LSF plus the diode array response to the approximately white light illuminating it.

While we do not know how much of the total background count rate as measured in the zero-sensitivity regions of our G130H spectra was scattered light, we do know that it was small compared to the first-order light everywhere longward of geocoronal Ly $\alpha$. In Figure 18 we plot the ratio of the adopted total background counts to the total observed number of counts in the object spectrum. Even at the shortest calibrated wavelengths, where the detector sensitivity is lowest, the contribution of the total background to the observed spectrum is $\$ 50 \%$. In this regime it is not necessary that we know precisely the relative contributions of the chargedparticle background and scattered-light background to the total background. A spectrum which is corrected assuming all of the measured background counts are due to the particle background is virtually indistinguishable from one where the excess background counts over the PODPS particle background model are assumed to be scattered light whose amplitude is constant along diodes (our adopted correction). The largest differences between the two extreme corrections were found to be $1 \%-2 \%$ in the first $15 \AA$ of the calibrated spectrum, and they fell off rapidly with increasing wavelength.

The much greater sensitivity in the G190H spectrum should render the effects of any scattered-light contribution insignificant for these data.

\section{APPENDIX B \\ INTERCALIBRATION OF THE G190H AND G130H SPECTRA}

To put the spectra obtained with the two gratings on the same wavelength scale, we attempted to measure the positions of the Galactic absorption lines. This was not a trivial task, since the Galactic absorption lines are very weak in this object, especially those which fall within the G190H spectra. We measured their equivalent widths to be approximately in the range $0.08-0.64 \AA$. In addition, these narrow spectral features were heavily smoothed by the broad PSF admitted by the large aperture. Assuming that the spectra in each grating are optimally aligned in wavelength, via the method described in $\S 2.3 .2$, we attempted to measure the positions of the absorption features in the mean spectrum of each grating. Because all of the absorption features are weak and lie upon broad emission lines, local fits to the spectra were required. We used the FOS G130H and G190H LSFs for the Galactic absorption lines, and Gaussian functions to fit the broad emission-line profiles. The resulting fits of regions least contaminated by broad emission lines indicated that the positions of the Galactic absorption lines lie within $\sim 0.5$ pixels of their vacuum wavelengths. In addition, the LSFs were good fits to the observed mean spectrum Galactic absorption-line profiles; thus, the mean spectra were not significantly blurred by errant zero-point wavelength corrections made in the individual spectra. 


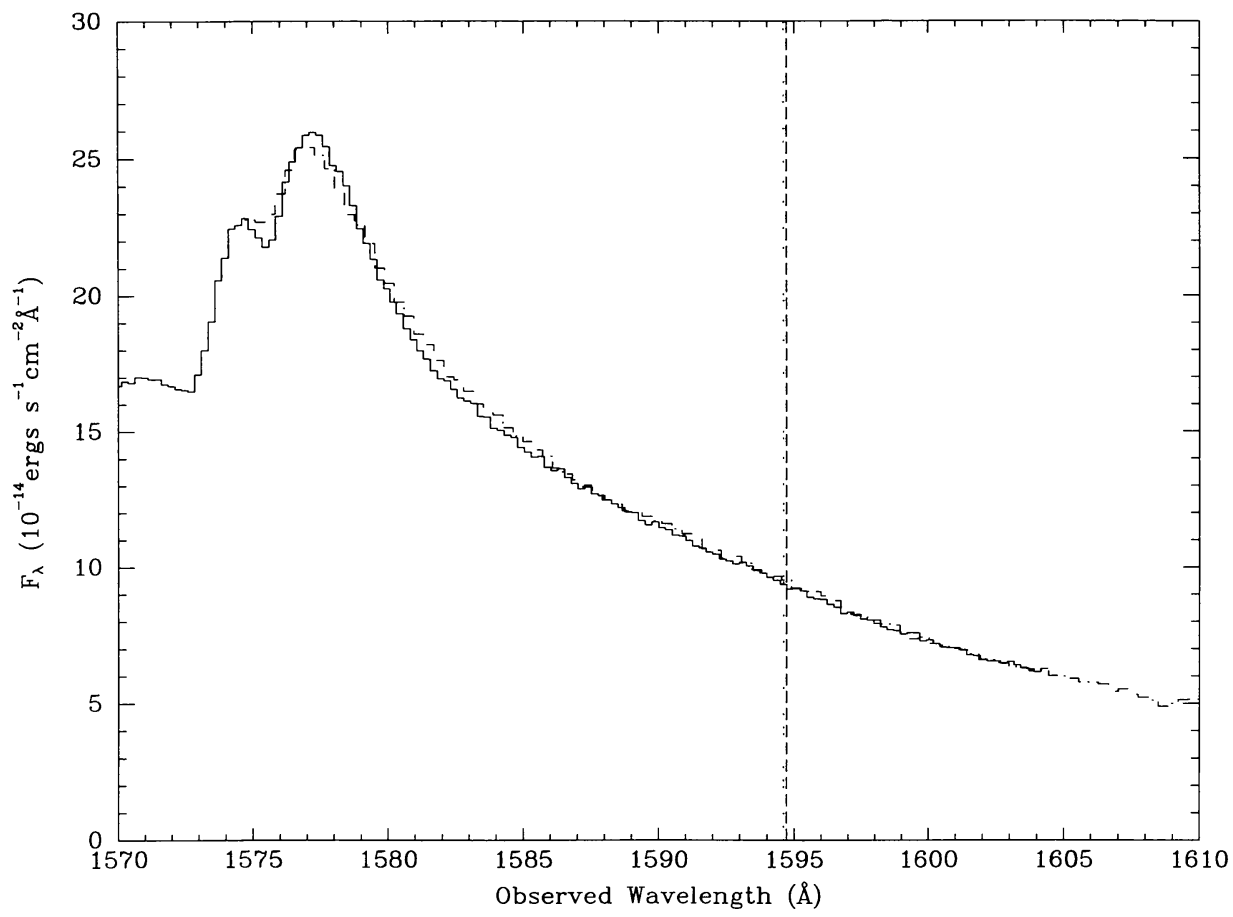

FIG. 19.-Mean G130H (solid line) and G190H (dot-dash line) spectra in the overlap region around the peak and red wing of the C IV emission line. The vertical lines designate the wavelengths where the two gratings were joined: 1594.60 and $1594.71 \AA$, respectively.

Next we compared the $\mathrm{G} 190 \mathrm{H}$ spectra with the $\mathrm{G} 130 \mathrm{H}$ spectra in the overlap region of the two gratings. This region spans the range 1574-1605 $\AA$ across the peak and red core of the C IV emission line. Figure 19 shows the mean G190H and G130H spectra in the overlap region. Unfortunately, the peak of the C IV emission line lies in the first few pixels of the G190H spectra where the signal-to-noise ratio is very low, and a cross-correlation of the C IV peak in the two gratings could not be done reliably to intercalibrate the two wavelength scales. Thus we were left with comparing the spectra along the core and wing of the $C$ Iv line in the overlap, where either a small shift in flux (typically at the $\sim 1 \%-2 \%$ level in either spectrum) or a small additive shift in wavelength (typically 1 pixel in eithe: spectrum) might produce a better alignment in some individual pairs of G190H and G130H spectra. Figure 19 illustrates these differences between the two sets of mean spectra in the overlap region. This figure typifies what occurred for individual epochs, in that the differences systematically "rippled" across the overlap region in this fashion. The mean fluxes, measured in the interval $1580-1600 \AA$, differed by $\$ 2.5 \%$ in all but a handful of epochs. We also note that a significant upward "blip" in the C IV profile, between 1595 and $1597 \AA$, of the JD 2,449,112 observation is mainly the result of a "blip" in the G190H spectrum rather than that of a large mismatch between the two spectra. The G130H spectrum does lie, on average, $2.7 \%$ below the G190H spectrum in the $1590-1600 \AA$ interval for this epoch.

There are several reasons why we made no attempt to reconcile the small differences between the two sets of grating spectra in the overlap region. First, the $\mathrm{G} 190 \mathrm{H}$ data do not have an arc comparison line which falls in the overlap region (the nearest is at $\sim 1621$ $\AA$ ), while the G130H data do. Extrapolation of the wavelength scale beyond this last arc line for the G190H data could cause a 1 pixel error in its local wavelength scale, as well as accompanying small errors $(\$ 1 \%)$ in the flux calibration. Second, the uncertainties in the FOS inverse-sensitivity functions are $\sim 1 \%$, but probably a bit larger at the ends of the spectra. The rippling in the differences within the overlap region of the mean $\mathrm{G} 130 \mathrm{H}$ and $\mathrm{G} 190 \mathrm{H}$ spectra is likely a manifestation of these two uncertainties. Third, the photometric repeatability of the FOS blue side for these two gratings is $1.4 \%(1 \sigma)$ for well-centered spectra (Lindler \& Bohlin 1994). Finally, photometric uncertainties, due to uncertainties in the placement of the spectral image upon the diode array, are wavelengthdependent and are at least as large as the differences quoted above (see Appendix D).

In constructing the combined spectrum, the $\mathrm{G} 130 \mathrm{H}$ spectrum was joined to the $\mathrm{G} 190 \mathrm{H}$ spectrum at pixels corresponding to wavelengths 1594.60 and $1594.71 \AA$, as indicated in Figure 19. The uncertainties in the wavelength and flux calibrations, as well as those in this joining procedure, will introduce small errors in the $\mathrm{C}$ IV broad emission line flux and profile.

\section{APPENDIX C KNOWN SOURCES OF PHOTOMETRIC ERROR IN THE FOS}

There are several known sources of photometric error in the FOS (Bohlin 1993a; Lindler \& Bohlin 1994 and references therein). Below we discuss separately eight known potential sources of photometric error that affect the FOS spectra.

1. Sensitivity degradation.-There has been a degradation in the blue-side sensitivity of $\sim 10 \%$ which occurred mainly between 1991 and 1992. This degradation has been tracked, and it seems to have leveled off since 1992. 
2. Throughput changes.- Long timescale throughput variations have occurred on account of changing telescope focus due to desorption, or "outgassing," of the optical telescope assembly. The amplitude of this effect is apparently damping out in time.

3. Long-term spectral image drift. - The spectral image of the electrons leaving the photocathode and landing upon the diode array has been drifting positionally along the FOS $Y$-axis in time since Science Verification (Koratkar \& Taylor 1993). Unfortunately, this drift was not noticed until 1993 June, after these observations had been completed. For this data set, the drift from optimal position was found to be about $+20 Y$-base units, or about $8 \%$ of the height of a diode ( $=256 Y$-base units), in the 2 years separating observations of the flux calibration star and observations of NGC 5548. Simulations show that a drift of the image of this magnitude from the optimal position at the center of the diode array produces a $1 \%$ (slightly wavelength-dependent) loss of light.

4. Short-term spectral image drift.-A shift in the position of the image on the diode array occurs due to GIMP. The deflection of the image, due to insufficient shielding of the instrument from the Earth's magnetic field, has components along and perpendicular to the diode array. The on-board GIMP correction is done in real time in both directions, and any residual photometric error due to this effect is expected to be small (less than $1 \%$ ) using the blue-side detector with the large (4"3 square) aperture.

5. Thermal-breathing effects.-A change in focus occurs due to "thermal breathing" of the secondary mirror support structures. Here the telescope focus changes and the image shifts slightly as various elements supporting the secondary mirror warp because of the rapid temperature changes as the telescope crosses the day/night terminator.

6. Thermal jitter.-Mispointing of the telescope occurs as a result of jitter a few minutes after crossing the day/night terminator. This jitter is the well-known thermal instability problem of the preservicing mission solar panels, and can last for a few minutes after onset.

7. Pointing errors. - In this program, the centering of the object in the aperture is accurate to about 0.175 (the mode of the distribution, about $32 Y$-base units) and 2 pixels along the FOS $Y$ - and $X$-directions, respectively, with a maximum expected excursion of a factor of $\sqrt{2}$ larger (about 0 ".25 [ $44 Y$-base units] and 2.8 pixels along the FOS $Y$-and $X$-directions). Because of the skewness of the pointing-error distribution, most pointings should have errors smaller than or equal to the mode value.

8. Filter-grating-wheel repeatability limitations. - The repeatability of the filter-grating-wheel position is accurate to about 13 $Y$-base units ( $0.073[1 \sigma]$ or about 0.8 pixels along the FOS $X$-direction).

The combined error of effects 1 and 2 is at the $\sim 10 \% \pm 5 \%$ level and is wavelength-dependent. Note, however, that problems 13 affect the data on timescales that are long compared with any important timescale for the NGC 5548 HST FOS campaign and are essentially systematic offsets which have been corrected in a post-pipeline recalibration. These problems do not affect the relative calibration of the NGC 5548 spectra and thus have no impact on any of our conclusions about variability during the course of the campaign.

Problems 4-6 are of more concern, since they occur on orbital timescales. As noted above, the on-board GIMP correction probably ensures that the effect of problem 4 is negligible. Problems 5 and 6 may have affected this data set to some extent. Their effects on the FOS photometry are not, as of this writing, as well modeled, but it is believed that their combined error could be at the $1 \%-3 \%$ level and is, of course, dependent on orbital position. This is consistent with the scatter remaining (1.4\% rms) after correcting for effects of problems 1-3 in well-centered FOS standard star calibration $\mathrm{G} 130 \mathrm{H}$ and $\mathrm{G} 190 \mathrm{H}$ data on the blue side.

Problems 7 and 8 appear to be semirandom in nature. When combined, these last two sources of error might result in $1 \%-4 \%$ photometric errors, assuming these $Y$-base offsets are centered about the position of the " $Y$-base drift," quoted above.

The manifestation of the errors induced by effects 5-7 in the NGC 5548 campaign data is influenced by our choice of the 4".3 aperture for the observations. Since the diode array rather than the aperture itself determines the effective edge of the aperture, offsets in the $Y$-direction lead to wavelength-dependent photometric errors rather than just loss of light. Although these errors include color terms, the effects are much less severe than they would have been if a smaller aperture had been used.

It is important to remember that since the spectral image is curved (covering $\sim 20-40 Y$-base units, depending on the grating) via the focusing of the electrons in a magnetic field, and given the amount of light which is contained within the broad wings of the PSF for the 4"3 3 1"4 effective aperture, any significant error in the positioning or a change in shape of the image on the diode array will produce wavelength-dependent flux calibration errors. In particular, a miscentering in a positive $Y$-base direction, as measured from the center of the diode array, loses more light from the blue end of the G130H spectrum and the red end of the G190H spectrum, while a miscentering in the negative $Y$-base direction loses more light from the overlap region of the two gratings. Because the G190H image is roughly twice as large in the FOS $Y$-direction as the G130H image, it will be somewhat more sensitive to miscenterings.

\section{APPENDIX D \\ PHOTOMETRIC ANOMALIES IN THE FOS DATA SET}

\section{D1. THE G130H "DROPOUTS"}

There were five epochs (FOS days 3, 7, 23, 24, and 33 = JD 2,449,099.04, 2,449,103.12, 2,449,118.99, 2,449,119.99, and $2,449,129.03$, respectively) where the G130H counts were anomalously low by $25 \%-65 \%$, with the loss of light highly wavelengthdependent. In the bottom panel of Figure 3 one can clearly see the sudden and drastic drop in counts for the G130H exposures for these five dates. The G190H exposures taken just one orbit earlier were unaffected. Two other effects of this error were also present. First, large wavelength shifts of 2-4 pixels ( $0.5-1$ diode $)$ were required to align these spectra with the rest. Second, the narrow peaks of the emission lines were missing, which indicates that the spectral resolution was lower for these exposures. This could occur if the 
diode array were only detecting the wings of the PSF and the LSF. This would indicate a large mispointing of the spectral image. We note that the large wings on the PSF due to primary-mirror aberrations ironically saved us from a complete loss of data on these dropout events. A properly focused image that moved off the edge of the diode array would have lost nearly all of the light for that observation.

Parallel wide-field camera (WFC) exposures were taken throughout much of the $H S T$ campaign. We thank R. Griffiths for kindly offering us information from the WFC observations which allowed a determination of the relative telescope pointing during the FOS campaign. Nearly all of the G130H observations and all of the G190H observations having parallel exposures had relative shifts ranging from a few hundredths to 0.2 in right ascension and declination. They are of the amplitude expected given our centering procedure. The remaining few G130H observations, corresponding to the G130H "dropout" observations, had large, $\sim 1$ ", deviations in declination and deviations in right ascension which were generally larger than those expected from pointing errors. Because the detector position angle lay near $0^{\circ}$ during the campaign, the offsets in declination are essentially offsets in the FOS $Y$-direction (producing photometric errors), and the offsets in right ascension nearly correspond to offsets in the $X$-direction (producing wavelength shifts). The transformation is a simple rotation of axes by an amount corresponding to the detector position angle. We found that the "dropouts" had displacements in the FOS $Y$-direction which corresponded to roughly half the height of the diode array $(0 " 7)$. These were accompanied by correspondingly large displacements in the FOS $X$-direction. The effects of these two displacements would be a substantial loss of light and a zero-point wavelength shift, just as observed. The cause of the large shifts in the telescope pointing for the "dropouts" is presently unknown, but the error must have occurred during the reacquisition of NGC 5548 after it emerged from Earth occultation just prior to the G130H exposure.

In principle, one might be able to convert shifts in right ascension and declination to FOS shifts in $Y$-base and $X$-base units (along the height and length of the diode array) and, by using the information in the PSF and LSF, calculate the photometric offsets. However, in practice the wings of these functions are not well determined, so that any correction which involves moving the core of the PSF off the diode array becomes unreliable. In addition, the actual position of the image on the diode array is made further uncertain via the nonrepeatability in the filter-grating-wheel. However, in the three instances where the loss of light was less than $50 \%$ (JD 2,449,099.04, JD 2,449,1 18.99, JD 2,444,119.99), the predicted loss of light, as derived from FOS throughput simulations, matched reasonably well with that derived via interpolation and use of the accompanying G190H exposure. This match is shown in Figure 20 for the first "dropout" G130H exposure. In this interpolation scheme we measured the mean flux in the $1585-1600 \AA$ band of the "dropout" $\mathrm{G} 130 \mathrm{H}$ and compared it with that in the accompanying, unaffected, G190H spectrum. We then scaled the "dropout" $\mathrm{G} 130 \mathrm{H}$ spectrum by this ratio. The simple scaling resulted in a fairly accurate recalibration of the C IV emission-line flux, but became an increasingly bad approximation at shorter wavelengths. To correct for the color dependency in the loss of light, we created a spectrum which is an interpolation of two good G130H spectra on either side of the "dropout" in time. This interpolation spectrum was then divided by the scaled "dropout" G130H spectrum, and a low-order polynomial was fitted through this

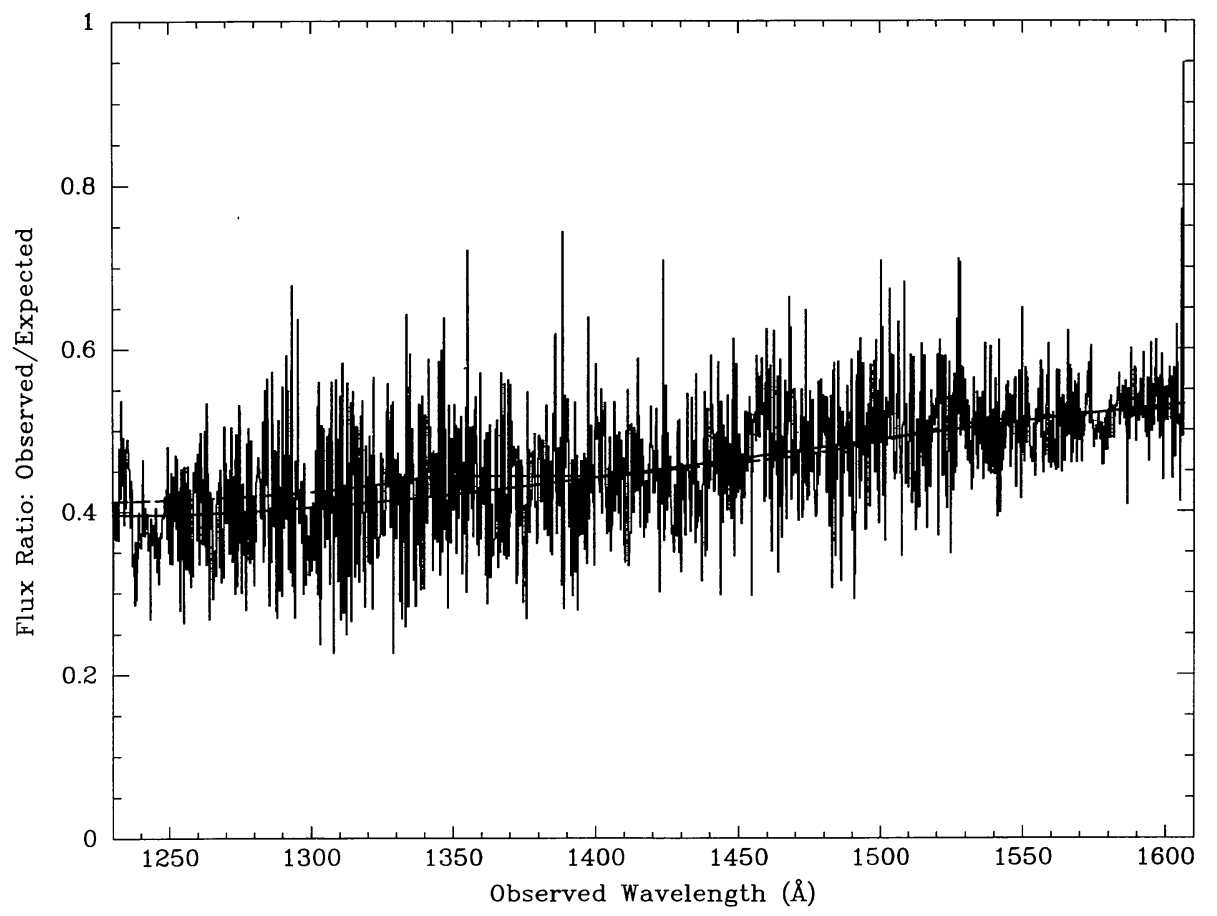

FIG. 20.-Spectrum of the ratio of the observed flux to the expected flux in the G130H "dropout" spectrum, JD 2,449,099. See text for explanation of how the expected spectrum was derived. The solid-line function is a low-order polynomial fit through the ratio, and is the adopted correction function for this G130H "dropout." The dashed-line function is the predicted ratio, based upon FOS simulations. 
ratio. This fit was subsequently used to correct the scaled "dropout" G130H spectrum for the color-dependent loss of light. This assumes that any intrinsic wavelength-dependent changes in the spectral flux occurred smoothly in time across the "dropout" exposure. This may not be correct, but for the smaller (less than 50\%) light losses this scheme matches the simulations well. We emphasize that the resulting "corrected" G130H spectrum is not a pure interpolation. Only the color was interpolated, the interpolation becoming less important (and thus less uncertain) in the region of the C IV emission line, where we have overlap information with the unaffected G190H spectrum.

\section{D2. THE "U-SHAPE ANOMALY"}

This anomaly strongly affected both gratings in four epochs (FOS days 18,20,21, and 34), and to lesser extents in at least two others (e.g., FOS days 35 and 36). It was characterized by two phenomena: $(a)$ it introduced abrupt features in the light curves of the continuum and the emission lines (i.e., some kind of correlated continuum/emission-line error was introduced), and $(b)$ ratios taken between the combined G130H and G190H spectra for these anomalous epochs and combined G130H and G190H spectra from other epochs appeared "U"-shaped, i.e., generally high on the ends and lower in the middle. A "U-shape" appeared only if the ratio included one of these "anomalous" spectra (other ratios appear monotonically rising or falling across the combined spectra). In the strongly affected spectra this "U-shape" amounted to a $\gtrsim 10 \%$ variation in flux from the ends of the combined G130H and $\mathrm{G} 190 \mathrm{H}$ spectrum to the middle, with the region near where the two spectra overlap least affected. In every strongly affected spectrum but one (day 34), the anomalous "U-shaped" spectrum had greater counts at all wavelengths than in neighboring observations. Day 34 lost counts in the middle of the combined spectra (i.e., near the overlap region). These shapes are characteristic of misplacements of the G130H and G190H spectral images upon the diode array. In all cases the effect was stronger in the G190H spectrum; this is expected, since the G190H spectral image has a larger extent in the FOS $Y$-direction. Summarizing from Appendix $\mathrm{C}$, the following are the well-understood sources of image misplacement and their expected $1 \sigma$ amplitudes in FOS $Y$-base units:

1. Telescope centering errors: $\pm 32 Y$-base units (maximum of \pm 44 ).

2. Filter-grating-wheel nonrepeatability: $\pm 13 Y$-base units.

3. Systematic $Y$-base drift of the image on diode array: $+20 Y$-base units.

It is conceivable that on certain occasions the combined effect of the two semirandom errors with the systematic " $Y$-base drift" resulted image centering errors large enough to produce the effects observed. In general, the "U-shapes" would have been better centered on the diode array (higher counts than neighboring spectra). As mentioned in Appendix $\mathrm{C}$, these miscentering errors might typically result in a few percent photometric error, although larger excursions could, and apparently did, occur.

As we had no way of independently determining what these excursions were, we could only attempt to remove the gross " $U$ shape" in the worst cases via broadband interpolation, as was done in the G130H "dropouts." This was done for both gratings for FOS days 18, 20, 21, 34, and for the G190H spectra only of FOS days 35 and 36. Generally, a low-order (typically third-order) polynomial was fitted through a ratio of the anomalous spectrum to an interpolated one, derived as a mean between two less affected spectra on either side.

This correction was attempted for only the most heavily affected spectra, as identified above. However, a casual glance at the light curves for various continua and emission lines across the entire spectrum reveals several other cases of abrupt 1 day excursions lying several statistical (Poisson counting) standard deviations away from neighboring points. This problem affected all spectra to some extent, more so at some wavelengths than at others.

\section{D3. "SUBGROUP VARIATIONS"}

The individual spectra were accumulated in several readouts (seven for the $\mathrm{G} 130 \mathrm{H}$ and five for the G190H exposures), which were examined separately for changes in the counting statistics during an exposure. These subgroups were of equal integration time subexposures (or readouts) of $62.50 \mathrm{~s} \mathrm{pixel}^{-1}$ for the $\mathrm{G} 130 \mathrm{H}$ data and $64.75 \mathrm{~s} \mathrm{pixel}^{-1}$ for the $\mathrm{G} 190 \mathrm{H}$ data. When the separate subgroups were compared, we often observed a pattern of increasing or decreasing counts during a single exposure (i.e., nonPoissonian variations), even after we had corrected for the small background contribution. These variations occurred in both gratings and had amplitudes of less than $\pm 1.5 \%$ about the mean for all cases except the G130H "dropouts," which had variations ranging over $\sim \pm 3 \%$ to $\pm 8 \%$ about the mean. The change in counts with time was moderately wavelength-dependent. The larger relative photometric variations occurring in exposures for which the image was placed near the edge of the diode array (i.e., for the G130H "dropouts") is consistent with an additional time- (or orbital-) dependent misplacement of the image upon the diode array for all epochs. This photometric error is similar in character and amplitude to what one might expect from the "thermal breathing" problem described in Appendix C. As of this writing, no reliable corrective model exists for these orbital or time-dependent photometric errors, and no corrections were attempted.

Ayres, T. R. 1994, FOS Instrument Sci. Rep. CAL/FOS-115 Beckert, D. C., \& Newberry, M. V. 1989, PASP, 101, 849

Blandford, R. D., \& McKee, C. F. 1982, ApJ, 255, 419

Bohlin, R. C. 1993a, private communication

- 1993b, in Proc. Workshop on Calibrating Hubble Space Telescope,

ed. J. C. Blades \& S. J. Osmer (Baltimore: STScI), 234

\section{REFERENCES}

Bohling, R. C. 1994, private communication

Bohlin, R. C., Harris, A. W., Holm, A. V., \& Gry, C. 1990, ApJS, 73, 413

Clavel, J. 1991, in Variability of Active Galactic Nuclei, ed. H. R. Miller \&

P. J. Wiita (Cambridge: Cambridge Univ. Press), 301

Clavel, J., et al. 1991, ApJ, 366, 64 (Paper I)

Crenshaw, D. M., \& Blackwell, J. H., Jr. 1990, ApJ, 358, L37 
Cunningham, C. C., \& Caldwell, J. J. 1993, in Proc. Workshop on Calibrating Hubble Space Telescope, ed. J. C. Blades \& S. J. Osmer (Baltimore: STScI), 199

Dietrich, M., et al. 1993, ApJ, 408, 416 (Paper IV)

Edelson, R. A., \& Krolik, J. H. 1988, ApJ, 333, 646

Evans, I. N. 1993, FOS Instrumentation Sci. Rep. CAL/FOS-104

I-I Ferland, G. J., Peterson, B. M., Horne, K., Welsh, W. F., \& Nahar, S. N. 1992, ApJ, 387, 95

Fitch, J. E., Hartig, G. F., Beaver, E. A., \& Hier, R. G. 1993, FOS Instrument Sci. Rep. CAL/FOS-098

Fitch, J. E., \& Schneider, G. 1993, FOS Instrument Sci. Rep. CAL/FOS099

Gaskell, C. M., \& Peterson, B. M. 1987, ApJS, 65, 1

Gaskell, C. M., \& Sparke, L. S. 1986, ApJ, 305, 175

Horne, K. 1986, PASP, 98, 609

Horne, K., Welsh, W. F., \& Peterson, B. M. 1991, ApJ, 367, L5

Johnson, H. L. 1966, ARA\&A, 4, 193

Junkkarinen, V., Beaver, E., Cohen, R., \& Lyons, R. 1991, FOS Instrument Sci. Rep. CAL/FOS-066

Kinney, A. L., \& Bohlin, R. C. 1993, FOS Instrument Sci. Rep. CAL/FOS103

Kinney, A. L., Bohlin, R. C., \& Neill, J. D. 1991, PASP, 103, 694

Koratkar, A. L., \& Taylor, C. 1993, FOS Instrument Sci. Rep. CAL/FOS116

Kriss, G. A. 1994, in Proc. Third Conf. on Astronomical Data Analysis Software and Systems, ed. D. Crabtree (San Francisco: ASP), in press

Krolik, J. H., Horne, K., Kallman, T. R., Malkan, M. A., Edelson, R. A., \& Kriss, G. A. 1991, ApJ, 371, 541
Lindler, D. J., \& Bohlin, R. C. 1994, FOS Instrument Sci. Rep. CAL/FOS125

Lyutyi, V. M. 1972, Astron. Zh., 49, 930

Maoz, D., et al. 1993, ApJ, 404, 576

Netzer, H., et al. 1990, ApJ, 353, 108

Nichols, J. S., Garhart, M. P., De La Peña, M. D., Levay, K. L., Stone, D. F., \& Turnrose, B. E. 1993, International Ultraviolet Explorer New Spectral Image Processing System Information Manual: Low Dispersion Data, Version 1.0

Penston, M. J., Penston, M. V., \& Sandage, A. 1971, PASP, 83, 783

Peterson, B. M. 1993, PASP, 105, 247

Peterson, B. M., et al. 1991, ApJ, 368, 119 (Paper II)

Peterson, B. M., et al. 1992, ApJ, 392, 470 (Paper III)

Peterson, B. M., et al. 1994, ApJ, 425, 622 (Paper VII)

Reichert, G. A., et al. 1994, ApJ, 425, 582 (Paper V)

Romanishin, W., et al. 1994, ApJ, submitted

Rosa, M. 1993, in Proc. Workshop on Calibrating Hubble Space Telescope, ed. J. C. Blades \& S. J. Osmer (Baltimore: STScI), 190 1994, private communication

Shull, J. M., \& Sachs, E. R. 1993, ApJ, 416, 536

Sirk, M., \& Bohlin, R. C. 1985, FOS Instrument Sci. Rep. CAL/FOS-013

Skillman, D. R., \& Patterson, J. 1993, ApJ, 417, 298

Smith, H. A., Matthews, J. M., Lee, K. M., Williams, J., Silbermann, N. A., \& Bolte, M. 1994, AJ, 107, 679

Stirpe, G. M., et al. 1994, ApJ, 425, 609 (Paper VI)

Weinstein, D., \& Carini, M. 1992, NASA IUE Newsletter, 49, 5

White, R. J., \& Peterson, B. M. 1994, PASP, 106, 879 